\title{
GENERALIZATION OF MIXED MULTISCALE FINITE ELEMENT METHODS WITH APPLICATIONS
}

\author{
A Dissertation \\ by \\ CHAK SHING LEE \\ Submitted to the Office of Graduate and Professional Studies of \\ Texas A\&M University \\ in partial fulfillment of the requirement for the degree of \\ DOCTOR OF PHILOSOPHY
}

$\begin{array}{ll}\text { Chair of Committee, } & \text { Yalchin Efendiev } \\ \text { Co-Chair of Committee, } & \text { Eduardo Gildin } \\ \text { Committee Members, } & \begin{array}{l}\text { Raytcho Lazarov } \\ \text { Jianxin Zhou }\end{array} \\ & \text { Emil Straube }\end{array}$

August 2016

Major Subject: Mathematics

Copyright 2016 CHAK SHING LEE 


\section{ABSTRACT}

Many science and engineering problems exhibit scale disparity and high contrast. The small scale features cannot be omitted in the physical models because they can affect the macroscopic behavior of the problems. However, resolving all the scales in these problems can be prohibitively expensive. As a consequence, some types of model reduction techniques are required to design efficient solution algorithms.

For practical purpose, we are interested in mixed finite element problems as they produce solutions with certain conservative properties. Existing multiscale methods for such problems include the mixed multiscale finite element methods. We show that for complicated problems, the mixed multiscale finite element methods may not be able to produce reliable approximations. This motivates the need of enrichment for coarse spaces.

Two enrichment approaches are proposed, one is based on generalized multiscale finite element methods (GMsFEM), while the other is based on spectral elementbased algebraic multigrid ( $\rho$ AMGe). The former one, which is called mixed GMsFEM, is developed for both Darcy's flow and linear elasticity. Application of the algorithm in two-phase flow simulations are demonstrated. For linear elasticity, the algorithm is subtly modified due to the symmetry requirement of the stress tensor.

The latter enrichment approach is based on $\rho$ AMGe. The algorithm differs from GMsFEM in that both of the velocity and pressure spaces are coarsened. Due the multigrid nature of the algorithm, recursive application is available, which results 
in an efficient multilevel construction of the coarse spaces.

Stability, convergence analysis, and exhaustive numerical experiments are carried out to validate the proposed enrichment approaches. Our numerical results show that the proposed methods are more efficient than the conventional methods while still being able to produce reliable solution for our targeted applications such as reservoir simulation. Moreover, the robustness of the mixed GMsFEM for linear elasticity with respect to the high contrast heterogeneity in Poisson ratio is evident from our numerical experiments. Lastly, our empirical results show good speedup and approximation by the proposed multilevel coarsening method. 


\section{ACKNOWLEDGMENTS}

First of all, I want to express my deepest gratitude to my advisor Prof. Yalchin Efendiev. He has been giving me continuous guidance and support on my research throughout my graduate studies in Texas A\&M, which is indispensable to the success of this thesis work. It was my pleasure to work with him; he always gave me inspiring directions in my research whenever I felt frustrated. Prof. Efendiev has been also very generous in supporting me for attending many workshops, conferences, and professional visits worldwide, which allowed me to exchange ideas with people having different areas of expertise and build up my professional network.

Secondly, I am really grateful to Prof. Eric Chung, who introduced me to the world of scientific research. He is always very patient in explaining his ideas to me, and gave me many useful advices in academic development. I really appreciate his generous support for my visits to the Chinese University of Hong Kong during my graduate study. The research work with him builds the foundation of this dissertation.

I am also very thankful to Prof. Panayot Vassilevski, whom I visited several summers as a student intern. I gained practical work experience from working with him and his team in Lawrence Livermore National Laboratory. He provided me interesting projects in scientific computing, which are beneficial to my research. In fact, part of this work was performed under the auspices of the U.S. Department of Energy by Lawrence Livermore National Laboratory under Contract DE-AC52- 
07NA27344. The IM release number for this manuscript is LLNL-TH-696037.

I also appreciate Department of Mathematics of Texas A\&M for providing me an awesome environment to develop my skills and knowledge. Moreover, I want to thank Prof. Eduardo Gildin, Prof. Raytcho Lazarov, Prof. Jianxin Zhou for serving on my dissertation committee, and thank Prof. Peter Howard and Prof. Joseph Pasciak for substituting in my preliminary and final exams respectively. Special thanks also go to my friends and fellow students for sharing with me the ups and downs during my $\mathrm{PhD}$ life.

Last but not least, I want to thank my wonderful parents and sister for the invaluable love they have been giving me all these years. That always charges me up and pushes me to move forward.

June 20, 2016

Chak Shing Lee 


\section{NOMENCLATURE}

$\begin{array}{ll}\mathbb{R}^{d} & d \text {-dimensional Euclidean space } \\ \Omega & \text { Simply-connected bounded open subset of } \mathbb{R}^{d} \\ p & \text { Pressure } \\ \boldsymbol{v} & \text { Velocity } \\ \boldsymbol{u} & \text { Displacement } \\ \underline{\sigma} & \text { Stress tensor } \\ h & \text { Characteristic length of a fine grid element } \\ H & \text { Characteristic length of a coarse grid element } \\ \mathscr{T}_{h} & \text { Fine mesh } \\ \mathscr{T}_{H} & \text { Coarse mesh } \\ \tau_{i} & i \text {-th fine grid element } \\ T_{i} & i \text {-th coarse grid element } \\ \mathscr{E}_{H} & \text { Set of coarse edges } \\ E_{i} & i \text {-th coarse edge } \\ \omega_{i}^{e} & i \text {-th coarse edge-based neighborhood } \\ \omega_{i}^{v} & \end{array}$




\section{TABLE OF CONTENTS}

Page

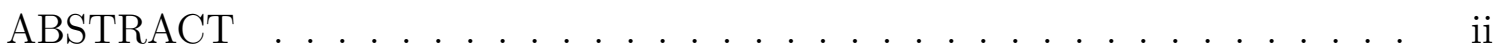

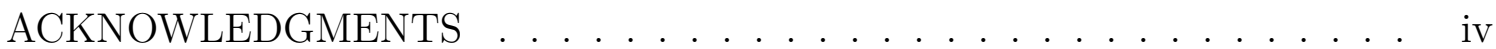

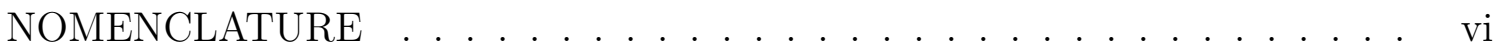

TABLE OF CONTENTS ............................. vii

LIST OF FIGURES . . . . . . . . . . . . . . . . . . . ix

LIST OF TABLES . . . . . . . . . . . . . . . . . . . xii

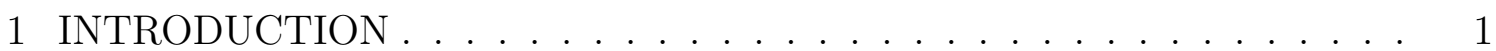

1.1 Mixed multiscale finite element methods and their limitations . . . . . 4

1.2 Enrichment of the multiscale coarse spaces . . . . . . . . . . . . . 10

2 MIXED GMSFEM FOR DARCY'S FLOW . . . . . . . . . . . . . . . 13

2.1 Model problem . . . . . . . . . . . . . . . . . . . . . . 13

2.2 The construction of multiscale basis functions . . . . . . . . . . . 16

2.2.1 Snapshot space . . . . . . . . . . . . . . . . . 17

2.2 .2 Offline space . . . . . . . . . . . . . . . . . 19

2.3 Optimization viewpoint of the basis functions . . . . . . . . . . 25

2.4 Postprocessing . . . . . . . . . . . . . . . . . . 27

2.5 Convergence of the mixed GMsFEM . . . . . . . . . . . . . . . . 28

2.6 Oversampling approach . . . . . . . . . . . . . . . . . 35

2.7 Numerical results . . . . . . . . . . . . . . . . . . . . . . . . . . . . 40

2.7.1 Single-phase flow . . . . . . . . . . . . . . . . . . . . . 41

2.7.2 Oversampling technique . . . . . . . . . . . . . . . 45

2.7.3 Single-phase flow and transport . . . . . . . . . . . . . . . 49

2.7.4 Two-phase flow and transport . . . . . . . . . . . . . 53

3 MIXED GMSFEM FOR PLANAR LINEAR ELASTICITY . . . . . . . . . 60

3.1 Model problem . . . . . . . . . . . . . . . . . . 61

3.2 Construction of the approximation space . . . . . . . . . . . . 63 
3.2.1 Edge-based basis functions for the stress tensor . . . . . . . . . 66

3.2.2 Vertex-based basis functions for the stress tensor . . . . . . . . . 69

3.2.3 Coarse space for displacement . . . . . . . . . . . . . . . . 71

3.3 Stability and convergence . . . . . . . . . . . . . . . . . 71

3.3.1 Snapshot projection . . . . . . . . . . . . . . . . 71

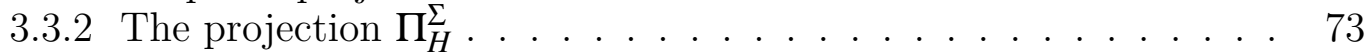

3.3 .3 Inf-sup stability . . . . . . . . . . . . . . . . . . . . 77

3.3 .4 Error estimate . . . . . . . . . . . . . . . . . . 80

3.4 Numerical results . . . . . . . . . . . . . . . . . . . . . . . . . . . 81

3.4.1 Snapshot error . . . . . . . . . . . . . . . . . . 82

3.4.2 Convergence against local enrichment . . . . . . . . . . . . . 83

3.4.3 Nearly incompressible material . . . . . . . . . . . . . . . . . . . 84

4 MUltilevel COARSE SPACE CONSTRUCTION BY $\rho$ AMGE . . . . . 87

4.1 The mixed finite element problem . . . . . . . . . . . . . . . . . . . 90

4.2 Coarse basis by the spectral AMGe method . . . . . . . . . . . . . . 92

4.2.1 Constructions of pressure space and velocity normal traces . . . 94

4.2.2 Construction of the velocity space . . . . . . . . . . . . . . . 98

4.2.3 Compatibility of the coarse pair of spaces . . . . . . . . . . . . 99

4.3 Approximation properties of the coarse pressure spaces . . . . . . . . 101

4.4 Stability properties and error analysis . . . . . . . . . . . . 103

4.5 Numerical experiments . . . . . . . . . . . . . . . . . 106

4.5 .1 2D examples . . . . . . . . . . . . . . . . 107

4.5.2 3D examples . . . . . . . . . . . . . . 116

5 EFFICIENT SOLVER FOR COARSE SADDLE POINT SYSTEMS . . . . 119

5.1 Hybridization . . . . . . . . . . . . . . . . . . . 122

5.2 Discussion . . . . . . . . . . . . . . . . . . . . . 124

5.3 Numerical examples . . . . . . . . . . . . . . . . . . . . . . . . 129

5.3 .1 Weak scaling . . . . . . . . . . . . . . . 130

5.3 .2 Strong scaling . . . . . . . . . . . . . . . . . . 132

5.3.3 Coarse saddle point problems . . . . . . . . . . . . . 135

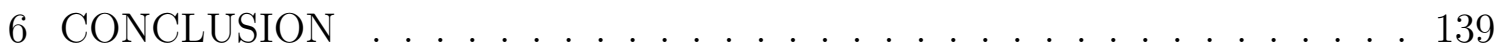

REFERENCES . . . . . . . . . . . . . . . . . . . . . 142 


\section{LIST OF FIGURES}

FIGURE

1.1 Illustration of a neighborhood $\omega_{i}=K_{i}^{+} \cup K_{i}^{-}$and an oversampled region $\omega_{i}^{+}$associated with the coarse edge $E_{i}$. . . . . . . . . . 6

1.2 Local coefficient $\kappa_{1}^{l o c}$ in a coarse neighborhood and the corresponding multiscale basis function $\phi_{i}^{1} \ldots \ldots \ldots$. . . . . . . . . 7

1.3 Local coefficient $\kappa_{2}^{l o c}$ in a coarse neighborhood and the corresponding multiscale basis function $\boldsymbol{\phi}_{i}^{2} \ldots \ldots \ldots$. . . . . . . 8

1.4 Channelized coefficient $\kappa_{\text {channel }} \ldots \ldots \ldots$. . . . . . . . 8

1.5 Unreliable approximation by mixed MsFEM when $\kappa=\kappa_{\text {channel }} \ldots$. . 9

$2.1 \quad$ First multiscale basis function $\boldsymbol{\psi}_{1}^{i}$ by mixed GMsFEM for the local coefficient in Figure 1.3(a). . . . . . . . . . . . . . . . . . . 22

2.2 Second multiscale basis function $\boldsymbol{\psi}_{2}^{i}$ by mixed GMsFEM for the local coefficient in Figure 1.3(a). . . . . . . . . . . . . . . . 22

2.3 Third multiscale basis function $\boldsymbol{\psi}_{3}^{i}$ by mixed GMsFEM for the local coefficient in Figure 1.3(a). . . . . . . . . . . . . . . . . . . . . . . . 23

$2.4 \quad$ Fine and coarse scale solutions when $\kappa=\kappa_{\text {channel }} . \ldots . . . . . .25$

2.5 Decay of $\log \left(1 / \lambda_{i}\right)$ for oversampling and non-oversampling. . . . . . 40

2.6 Three permeability fields in the numerical experiments. . . . . . . . 40

2.7 Inverse of eigenvalue $(1 / \lambda)$ behavior for the two spectral problems. 44

2.8 Saturation solution obtained by using $v_{f}$ in $(2.40) \ldots . . . . \quad 51$

2.9 Saturation solution obtained by using $v_{o}(10 \times 10$ coarse grid, 1 basis

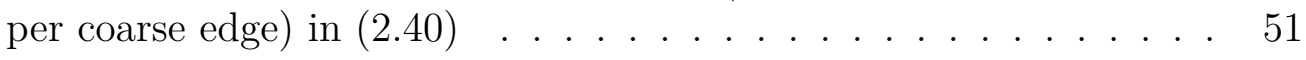

2.10 Saturation solution obtained by using $v_{o}(10 \times 10$ coarse grid, 3 basis

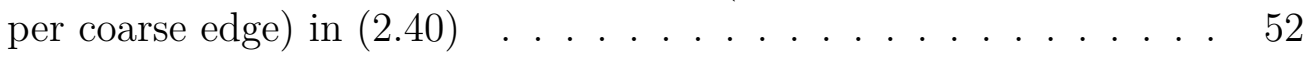


2.11 Saturation solution obtained by using $v_{o}(10 \times 10$ coarse grid, 5 basis per coarse edge) in $(2.40)$. . . . . . . . . . . . . . . 52

2.12 Saturation solution obtained by using $v_{f}$ in $(2.41) \ldots \ldots$. . . . 54

2.13 Saturation solution obtained by using $v_{o}(10 \times 10$ coarse grid, 1 basis

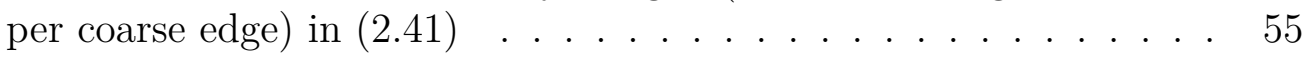

2.14 Saturation solution obtained by using $v_{o}(10 \times 10$ coarse grid, 3 basis

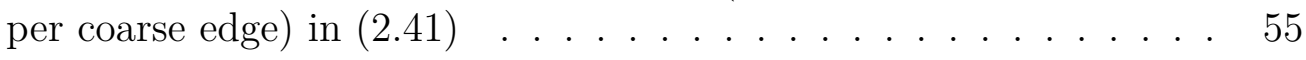

2.15 Saturation solution obtained by using $v_{o}(10 \times 10$ coarse grid, 5 basis

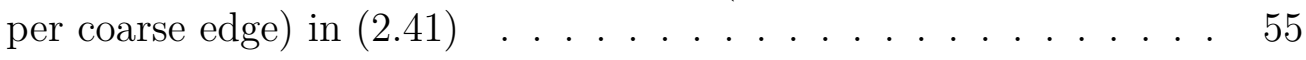

2.16 Saturation solution obtained by using $v_{f}$ in $(2.41) \ldots \ldots$. . . . 56

2.17 Saturation solution obtained by using $v_{o}(11 \times 11$ coarse grid, 1 basis

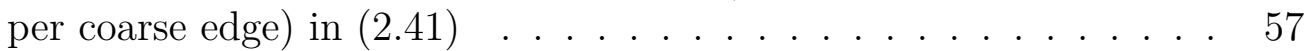

2.18 Saturation solution obtained by using $v_{o}(11 \times 11$ coarse grid, 3 basis

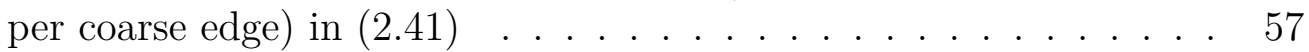

2.19 Saturation solution obtained by using $v_{o}(11 \times 11$ coarse grid, 5 basis

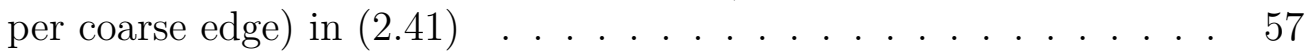

2.20 Water cut at producer for the two-phase flow example. . . . . . . 58

3.1 Examples of an edge-based coarse neighborhood $\omega_{i}^{e}=K_{j} \cup K_{l} \cup E_{i}$ associated with the coarse edge $E_{i}$ and a vertex-based coarse neighborhood $\omega_{j}^{v}$ associated with the coarse vertex $V_{j} \ldots \ldots . . . .64$

3.2 Error plot of the mixed GMsFEM approximation of the stress tensor relative to fine scale solution . . . . . . . . . . . . . . . 83

3.3 Top: $\sigma_{11}$, middle: $\sigma_{22}$, bottom: $\sigma_{12}$, left: fine solution $(\operatorname{dim}=50401)$, middle: coarse solution $(\operatorname{dim}=762)$, right: coarse solution $(\operatorname{dim}=1103) . \ldots \ldots \ldots$

4.1 Top layer of the SPE10 model and the full 3D model. . . . . . . . . 107 
4.2 Magnitudes of velocity solutions in Experiment 1. . . . . . . . . 109

4.3 Pressure solutions in Experiment 1. . . . . . . . . . . . 110

4.4 Velocity solutions on different levels in Experiment 4. . . . . . . . . 113

4.5 Pressure solutions on different levels in Experiment 4. . . . . . . . . 114

4.6 Agglomerates in each level in Experiment 4. To better visualize agglomerates we have applied a coloring algorithm. On level 2 (middle) and level 3 (right), each agglomerate consists of agglomerates from the previous (finer) level: we artificially separate the agglomerates coming from the previous level for visualization purposes. . . . . . . 114

4.7 Velocity basis by different number of level constructions. . . . . . . 115

4.8 Pressure basis by different number of level constructions. . . . . . . 116

4.9 The Egg model. . . . . . . . . . . . . . . . . 117

4.10 Magnitudes of velocity solutions in Experiment 6. . . . . . . . 118

5.1 Initial mesh for the $R T_{2}$ weak scaling test case. Blue region indicates

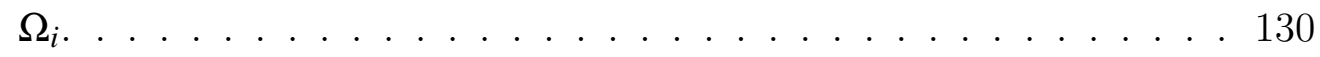

5.2 Weak scaling comparisons between hybridization (red dotted line) and ADS (blue solid line) . . . . . . . . . . . . 133

5.3 The mesh for the Crooked Pipe problem (left). A dense layer of highly stretched elements (right) has been added to the neighborhood of the material interface in the exterior subdomain in order to resolve the physical diffusion. . . . . . . . . . . . . . . . 134

5.4 Strong scaling comparison between hybridization (red dotted line) and ADS (blue solid line). Black dotted line indicates perfect scaling 136 


\section{LIST OF TABLES}

TABLE $\quad$ Page

2.1 Convergence of the offline solution, $\kappa=\kappa_{1}, n=200$ and $N=10 \quad$. 43

2.2 Convergence of the offline solution, $\kappa=\kappa_{1}, n=200$ and $N=20 \quad$. 43

2.3 Convergence of the offline solution, $\kappa=\kappa_{2}, n=200$ and $N=10 \quad . \quad 43$

2.4 Convergence of the offline solution, $\kappa=\kappa_{2}, n=200$ and $N=20 \quad$. 44

2.5 Comparison of velocity and postprocessed velocity, $\kappa=\kappa_{1}, n=200$ and $N=10$. . . . . . . . . . . . . . . . . . . . . . . . . . . . . . . . . . . . . . . . 45

2.6 Convergence of the offline solution using the curl-based spectral problem, $\kappa=\kappa_{1}, n=200$ and $N=10 \ldots . . . . . . .446$

2.7 Comparison of the 4 cases (relative velocity error w.r.t. fine scale solution), $\kappa=\kappa_{\text {per }}, n=200, N=10 \ldots \ldots$. . . . . . 47

2.8 Comparison of the 4 cases (relative velocity error w.r.t. fine scale solution), $\kappa=\kappa_{1}, n=200, N=10 \ldots \ldots$. . . . . . 47

2.9 Use of randomized boundary conditions in oversampling technique (relative velocity error w.r.t. fine scale solution), $\kappa=\kappa_{p e r}, n=200$, $N=10 \ldots \ldots \ldots \ldots$. . . . . . . . . . . . . . . . . . 48

2.10 Use of randomized boundary conditions in oversampling technique (relative velocity error w.r.t. fine scale solution), $\kappa=\kappa_{1}, n=200$,

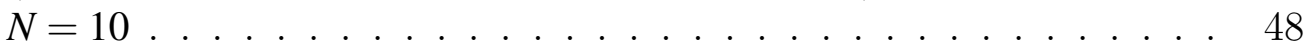

2.11 Two-phase flow example timing using different methods. . . . . . . 59

3.1 Experiment set 2. . . . . . . . . . . . . . . 84

3.2 Experiment set 3, high Poisson's ratio only in red region. . . . . . . 86

3.3 Experiment set 4, high Poisson's ratio in both regions. . . . . . . . 86 
4.1 Experiment 1 with various $\theta$. We have imposed an upper bound on the number of eigenvectors to be used (we let $k=10$ ). For the fine-grid level $\operatorname{dim}\left(\boldsymbol{V}_{h}\right)=26680$ and $\operatorname{dim}\left(Q_{h}\right)=13200$.

4.2 Experiment 1 with various $k$. For the fine-grid level, $\operatorname{dim}\left(\boldsymbol{V}_{h}\right)=$ 26680 and $\operatorname{dim}\left(Q_{h}\right)=13200 \ldots \ldots \ldots \ldots$

4.3 Performance of $\rho$ AMGe upscaling in Experiment 2. C.F. $=$ coarsening factor. For the fine-grid level, $\operatorname{dim}\left(\boldsymbol{V}_{h}\right)=106160$ and $\operatorname{dim}\left(Q_{h}\right)=$ 52800 .

4.4 Performance of $\rho$ AMGe upscaling in Experiment 3. FEO $=$ finite element order. . . . . . . . . . . . . . . . . 111

4.5 Performance of the $\rho$ AMGe upscaling in Experiment 4. For the fine-grid level, $\operatorname{dim}\left(V_{h}\right)=106160$ and $\operatorname{dim}\left(Q_{h}\right)=52800 \ldots \ldots 112$

4.6 Performance of the $\rho$ AMGe upscaling in Experiment 5. For the fine-grid level, $\operatorname{dim}\left(\boldsymbol{V}_{h}\right)=106160$ and $\operatorname{dim}\left(Q_{h}\right)=52800 \ldots \ldots 113$

4.7 Coarsening timings and errors by 2-, 3-, and 4-level hierarchies. . . 115

4.8 Performance of $\rho$ AMGe upscaling in Experiment 7. For the fine-grid level we have $\operatorname{dim}\left(V_{h}\right)=59205$ and $\operatorname{dim}\left(Q_{h}\right)=18553 \ldots \ldots \ldots 117$

5.1 Time to solution in seconds: $R T_{0}$ on tetrahedral meshes, the corresponding number of PCG iterations are the reported in the brackets. 131

5.2 Time to solution in seconds: $R T_{2}$ on tetrahedral meshes, the corresponding number of PCG iterations are the reported in the brackets. 132

$5.3 \quad$ Strong scaling test, original problem size: $2,805,520 \ldots \ldots \ldots \ldots$

5.4 Timing of each component of the new solver. . . . . . . . . 135

5.5 The numbers in the bracket is the $\mathrm{CG}$ iteration count. For the fine-grid level, $\operatorname{dim}\left(\boldsymbol{V}_{h}\right)=3,403,000$ and $\operatorname{dim}\left(Q_{h}\right)=1,122,000$. The solution time for the fine scale problem is about 61 seconds. . . . . 138 


\section{INTRODUCTION}

Many science and engineering problems exhibit scale disparity and high contrast. The small scale features can affect the macroscopic behavior of the problems one way or the other. Typical examples include modeling of composite materials, flows in porous media, and climate models of the globe. If one attempts to resolve all the scales at once, the problems can be prohibitively expensive to be solved even by the computing power nowadays.

Efficient methods for problems that are multiscale in nature have been a popular research topic in the past few decades. Instead of solving the problems directly in full resolution, a more common approach is to reduce the dimension of the models while preserving a certain acceptable accuracy. Along this direction, many multiscale or upscaling methodologies have been proposed over the years.

For instance, homogenization $[19,45]$ is one of the classic upscaling methods which mainly handles periodic coefficients. The general purpose of homogenization is to "homogenize" a heterogeneous coefficient by some averaging methods. Applicability of homogenization is limited by the assumption on coefficient (e.g. periodicity) and the geometry of the problem under consideration.

In the past two decades, various numerical homogenization methods were intro-

duced. The variational multiscale method [47] proposed by Hughes et al. decomposes the degrees of freedom (dofs) into two groups; one group is the coarse degrees of free- 
dom which is responsible for global coupling while the other group can be further decomposed into independent local degrees of freedom. Since the degrees of freedom in the second group is decoupled, the original problem is decomposed into small local subproblems which can be solved independently. These local problems are then coupled by the degrees of freedom in the first group through a global coarse problem. The coarse degrees of freedom are assumed to be in certain patterns (e.g. linear along subdomain interface). Such assumptions can greatly affect the accuracy of the method.

Another numerical homogenization method is the heterogeneous multiscale methods [31,32] proposed by E and Engquist, which assume that the homogenized problem of the original one is in a certain legitimate form. The homogenized problem is then solved by standard methods (e.g. finite element methods) on a coarse mesh. When assembling the discrete problems, local problems need to be solved and the solutions of local problems are used in the numerical integration so that local microscopic features are taken into account in the homogenized problem. Heterogeneous multiscale methods are particularly suitable when the information of the media is only available in some local representative volumes.

Besides variational multiscale methods and heterogeneous multiscale methods, multiscale finite element methods are also very popular among all the multiscale methods. Multiscale finite element methods were first introduced by Hou and Wu in 1997 [46], and further developed by several other authors [38, 37, 29, 22]. Con- 
ventional finite element methods use piecewise polynomials as basis functions of the approximation space. In contrast, the basis functions in multiscale finite element methods are constructed by solving local problems with prescribed (artificial) boundary conditions. Since these basis functions carry local information of the coefficient, they provide a better representation of the solution. It can be shown that in some situations, multiscale finite element methods coincide with the variational multiscale methods and heterogeneous multiscale methods [37].

Multiscale finite volume methods $[50,66,60,70]$ developed by Jenny et al. form another well-known class of multiscale methods which mainly aim at subsurface flow applications. Similar to multiscale finite element methods, multiscale finite volume methods also construct basis function by solving local problems. However, a distinct feature of multiscale finite volume methods is that, basis functions are built on a dual coarse mesh. Such a construction allows multiscale finite volume methods to produce mass conservative solutions to multiscale problems, hence the name multiscale finite volume.

Recently, Efendiev et al. introduced the generalized multiscale finite element methods (GMsFEM) [33], which generalize the multiscale finite element methods by using more local basis functions. The multiscale approximation space in GMsFEM is formed by the span of solutions of local spectral problems, as oppose to solutions of local boundary value problems in the case of multiscale fintie element methods. The construction of the multiscale approximation space is split into three stages: the 
snapshot space, the offline space, and the online space. The enriched approximation space renders GMsFEM to produce solutions with a smuch better accuracy for a broader class of problems (e.g. problems without scale separation).

A special variant of multiscale finite element methods is the mixed multiscale finite element methods $[20,2]$, which produce solutions with certain conservative properties (e.g. mass, energy) on the coarse mesh. The main purpose of this dissertation is to generalize the mixed multiscale finite element methods (following the framework of GMsFEM and $\rho$ AMGe) to allow more accurate approximations. As a motivation, we are going to review the mixed multiscale finite element methods and discuss their limitations in the next section.

1.1 Mixed multiscale finite element methods and their limitations

Consider the following system of partial differential equations

$$
\begin{aligned}
\boldsymbol{\kappa}^{-1} \boldsymbol{v}+\nabla p=0 \quad \text { in } \quad & \Omega, \\
\operatorname{div}(\boldsymbol{v})=f & \text { in } \quad \Omega, \\
\boldsymbol{v} \cdot \boldsymbol{n}=0 & \text { on } \quad \partial \Omega
\end{aligned}
$$

where $\kappa$ is a heterogeneous coefficient. We will see later that this system governs the motion of Darcy's flow. To describe the mixed multiscale finite element methods for the model problem (1.1), we first introduce the notion of fine and coarse grids. For the sake of simplicity, we will assume a 2D problem in the following discussion; the extension of the algorithm to $3 \mathrm{D}$ problems is straight forward. We let $\mathscr{T}_{H}$ be a usual conforming partition of the computational domain $\Omega$ into finite elements 
(triangles, quadrilaterals, tetrahedrals, etc.), called coarse-grid blocks, where $H>0$ is the coarse mesh size. We refer to this partition as the coarse grid and assume that each coarse-grid block is partitioned into a connected union of fine-grid blocks, which are conforming across coarse-grid edges. The fine grid partition will be denoted by $\mathscr{T}_{h}$, which by definition is a refinement of $\mathscr{T}_{H}$. We use $\mathscr{E}_{H}:=\bigcup_{i=1}^{N_{e}}\left\{E_{i}\right\}$ (where $N_{e}$ is the number of coarse edges) to denote the set of all edges of the coarse mesh $\mathscr{T}_{H}$, and $\mathscr{E}_{H}^{0}$ to denote the set of all interior coarse edges. We also define the edge-based coarse neighborhood $\omega_{i}^{e}$ corresponding to the coarse edge $E_{i}$ as the union of all coarse-grid blocks sharing the edge $E_{i}$, namely,

$$
\omega_{i}=\bigcup\left\{K_{j} \in \mathscr{T}_{H} ; \quad E_{i} \in \partial K_{j}\right\}
$$

See Figure 1.1 for an example of an edge-based coarse neighborhood, where the coarse-grid edges are denoted by black lines and the fine-grid edges are denoted by grey lines. In what follows, we will drop the superscript $e$ in $\omega_{i}^{e}$ when there is no confusion.

The unknown $p$ is approximated in the space of piecewise constant functions with respect to the coarse grid $\mathscr{T}_{H}$. The approximation space for $v$ is constructed locally as follows. For each coarse edge $E_{i}$, we solve the local boundary value problem

$$
\begin{aligned}
\boldsymbol{\phi}_{i} & =\kappa \nabla \xi_{i} & & \text { in } \omega_{i} \backslash E_{i} \\
\nabla \cdot \boldsymbol{\phi}_{i} & = \pm 1 /\left|K_{i}^{ \pm}\right| & & \text {in } \omega_{i} \backslash E_{i} \\
\boldsymbol{\phi}_{i} \cdot \boldsymbol{n} & =0 & & \text { on } \partial \omega_{i} \\
\boldsymbol{\phi}_{i} \cdot \boldsymbol{m}_{i} & =1 /\left|E_{i}\right| & & \text { on } E_{i}
\end{aligned}
$$




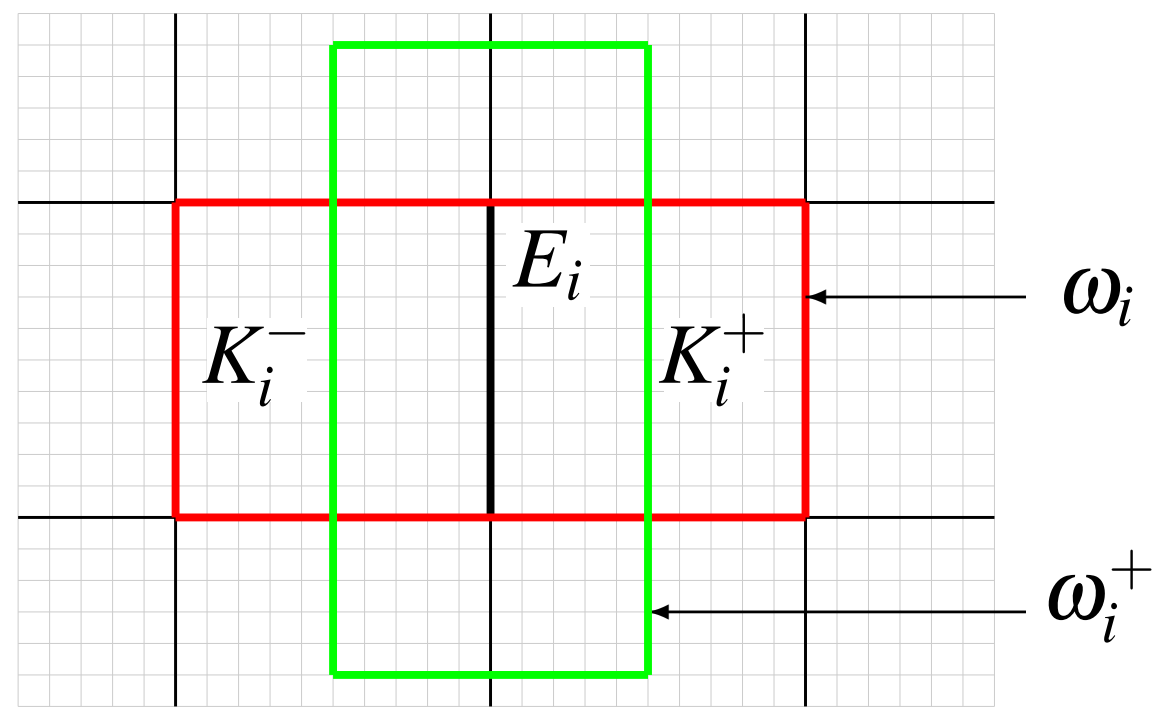

Figure 1.1: Illustration of a neighborhood $\omega_{i}=K_{i}^{+} \cup K_{i}^{-}$and an oversampled region $\omega_{i}^{+}$associated with the coarse edge $E_{i}$.

We remark here that if $\kappa$ is constant in $\Omega, \boldsymbol{\phi}_{i}$ coincides with the Raviart Thomas basis function. Hence, mixed multiscale finite element methods are generalization of the conventional mixed finite element methods. As an illustration of how the mixed multiscale finite element methods work, let us look at an example of coefficient $\kappa_{1}^{l o c}$ locally in a coarse neighborhood $\omega_{i}$ and the corresponding basis function $\boldsymbol{\phi}_{i}$ in Figure 1.2. The coefficient $\kappa_{1}^{l o c}$ has a smaller value in the blue regions. For Darcy's flow, $\kappa$ refers to the permeability field, which is a measure of how fast of a fluid flow through a certain region due to a given pressure difference. A fluid tends to flow through a high permeability region. Hence, we expect the global velocity solution not much going into the blue regions. As we can see, this local feature is well captured by the multiscale basis functions $\boldsymbol{\phi}_{i}^{1}$. In fact, it is obvious from the streamline of $\boldsymbol{\phi}_{i}^{1}$ that, the vector field tends to avoid going into the blue regions. Since $\boldsymbol{\phi}_{i}^{1}$ is a basis 


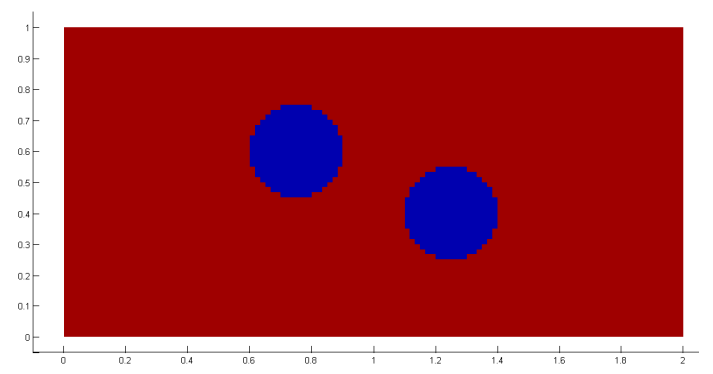

(a) Local coefficient $\kappa_{1}^{l o c}$

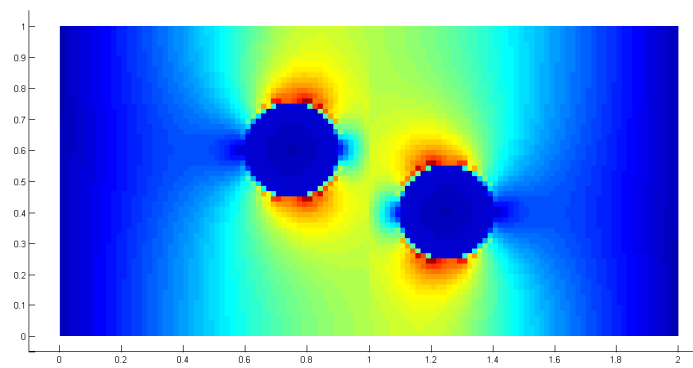

(b) Magnitude of $\boldsymbol{\phi}_{i}^{1}$

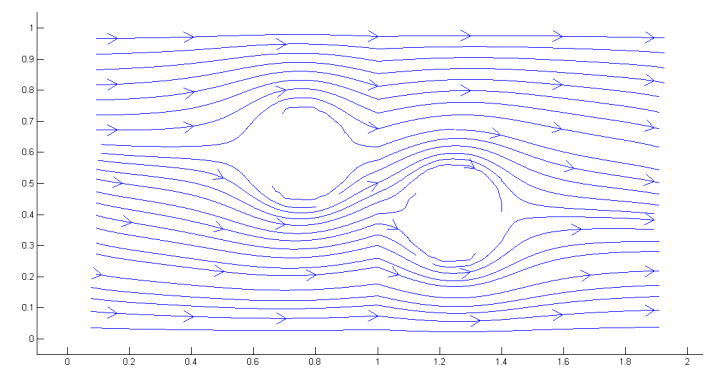

(c) Streamline of $\boldsymbol{\phi}_{i}^{1}$

Figure 1.2: Local coefficient $\kappa_{1}^{l o c}$ in a coarse neighborhood and the corresponding multiscale basis function $\boldsymbol{\phi}_{i}^{1}$.

function of the approximation space, this local feature will also be reflected in the multiscale solution.

Now, let us look at a slightly different coefficient $\kappa_{2}^{l o c}$ and the corresponding basis function $\boldsymbol{\phi}_{i}^{2}$ in Figure 1.3. For this second example, we can see from the streamline of $\boldsymbol{\phi}_{i}^{2}$ that the vector field still goes into the blue regions. The main difference between $\kappa_{1}^{l o c}$ and $\kappa_{2}^{l o c}$ is that, the heterogeneity in $\kappa_{2}^{l o c}$ lies on the coarse edge $E_{i}$. Since the definition of $\boldsymbol{\phi}_{i}$ assumes constant normal flux across $E_{i}$, the heterogeneity in $\kappa_{2}^{l o c}$ cannot be well-reflected in the $\boldsymbol{\phi}_{i}$, as illustrated in Figure 1.3. Moreover, for complex coefficients like the one shown in Figure 1.4, where we can 


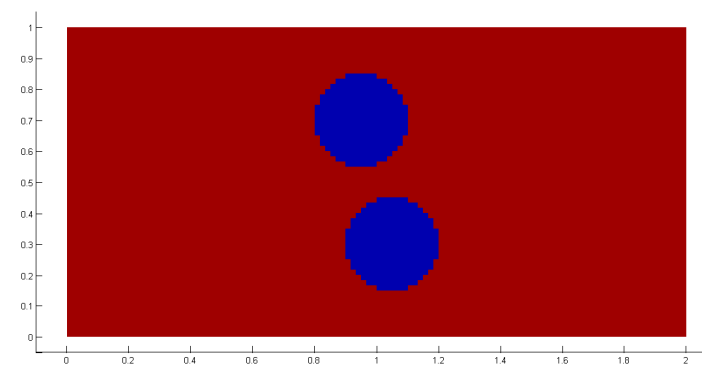

(a) Local coefficient $\kappa_{2}^{l o c}$

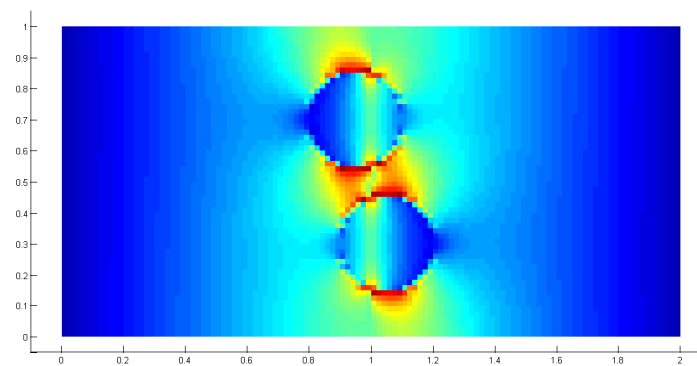

(b) Magnitude of $\boldsymbol{\phi}_{i}^{2}$

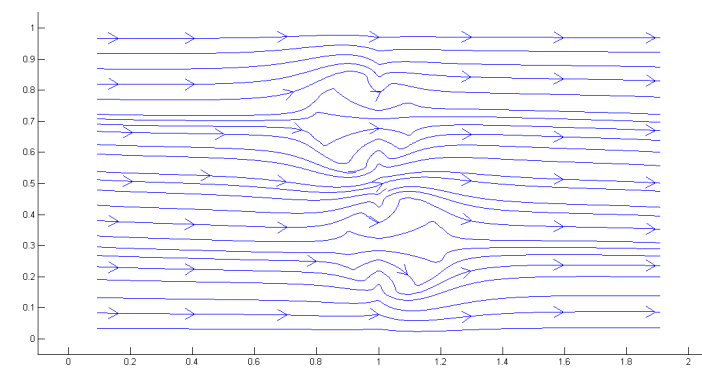

(c) Streamline of $\boldsymbol{\phi}_{i}^{2}$

Figure 1.3: Local coefficient $\kappa_{2}^{l o c}$ in a coarse neighborhood and the corresponding multiscale basis function $\boldsymbol{\phi}_{i}^{2}$.

see some channel regions, mixed multiscale finite element methods cannot produce good approximations. This is an example of coefficients without scale separation.

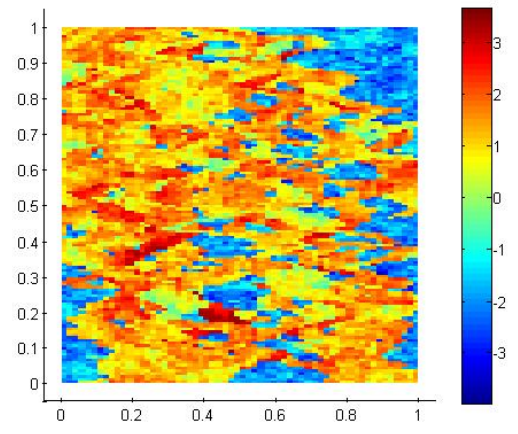

Figure 1.4: Channelized coefficient $\kappa_{\text {channel }}$. 
In Figure 1.5, we also plot out the reference solution for this coefficient obtained by solving (1.1) using conventional mixed finite element methods on a fine grid, and the coarse scale solution obtained by mixed multiscale finite element methods on a coarse grid. Obviously the coarse scale approximation by the mixed multiscale finite element methods is very different from the reference solution. In fact, the relative error of the coarse scale approximation is greater than $100 \%$, which clearly indicates that we cannot trust the coarse scale approximation at all. For this coefficient, one basis per coarse neighborhood is not enough to represent the complex heterogeneity.

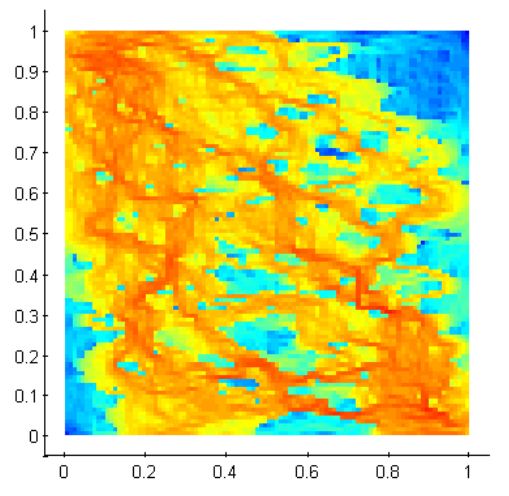

(a) Reference sol'n (dim=29799)

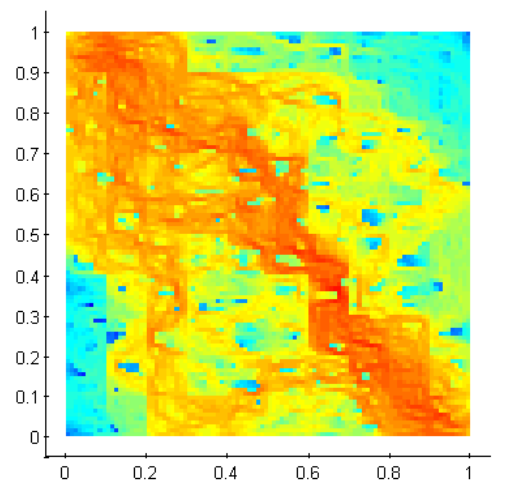

(b) Mixed MsFEM sol'n $(\operatorname{dim}=279)$

Figure 1.5: Unreliable approximation by mixed MsFEM when $\kappa=\kappa_{\text {channel }}$. 


\subsection{Enrichment of the multiscale coarse spaces}

We have shown in the previous section that, for some more complicated coefficients, traditional numerical homogenization methods like mixed multiscale finite element methods often cannot produce accurate approximation to the fine grid solution. To better capture the heterogeneity in the coefficient, the generalized multiscale finite element methods (GMsFEM) were proposed recently by Efendiev at el. [33].

Let us suppose that some informations (e.g. spatial heterogeneity pattern, probability distribution) of the coefficient are given. GMsFEM builds multiscale basis functions on a coarse grid in several stages. First, a snapshot space is constructed such that it can make very good approximation to the fine scale function space. One can either take the local snapshot space to be the local fine scale function space, or construct the local snapshot space by solving local boundary value problems. Next, local offline space is obtained through spectral decomposition of the local snapshot space. This step involves solving local eigenvalue problems. Normally the offline space has a much smaller dimension than the snapshot space because only a few eigenvectors will be taken to generate the offline space. Up to now everything can be done offline, which means these steps can be done before the actual simulation starts. In fact, if the coefficient does not depend on parameters, the offline space is the final coarse scale approximation space. However, there are situations when the coefficient does depend on some parameters. For example, in uncertainty quantifications, the coefficients depend on certain probability distribution; in time-dependent problems, 
the coefficient may depend on time. In such circumstances, a online space will be generated by performing spectral decomposition to the offline space. Although this last step is implemented online, it is computationally not expensive since the dimension of the offline space is much smaller than the fine scale approximation space.

In this dissertation, we will study the enrichment of the multiscale coarse spaces for mixed finite element problems. Two approaches are studied, namely, mixed GMsFEM and $\rho$ AMGe for mixed problems. The resulting multiscale methods are generalizations of the mixed multiscale finite element methods.

The mixed GMsFEM refers to the variant of GMsFEM that handles problems in mixed form. Such problems have more than one unknown, and the discrete version of the problems are usually saddle point systems. Mixed GMsFEM constructs coarse approximation spaces for all the unknowns of the problems, as oppose to GMsFEM originally proposed in [33] that considers problems with only one known. Our discussion will be focusing on the construction of snapshot space and offline space, construction of online space should be straight forward.

In the Section 2 and 3, we are going to discuss in detail the mixed GMsFEM for systems of partial differential equations describing the Darcy's flow [24] and linear elasticity, respectively. In mixed GMsFEM, only one of the coarse approximation spaces is enriched; the other is held fixed. On the other hand, in Section 4, we will discuss how to construct multiscale coarse spaces for mixed finite element problems 
within the $\rho$ AMGe framework. Such a framework allows coarsening of both of the approximation spaces. Moreover, the AMG nature of the algorithm allows recursive application, which results in a multilevel method. A multilevel construction can greatly reduce the construction time of the multiscale coarse spaces. 


\section{MIXED GMSFEM FOR DARCY'S FLOW}

Because of the scale disparity in the material properties (e.g. porosity and permeability), resolving all the scales of the flows in porous media can be prohibitively expensive. For this reason, the geological model is oftentimes upscaled to a computationally feasible model for simulation purpose. In this section, we will discuss the mixed generalized multiscale finite element methods (GMsFEM), which can be considered as a type of numerical upscaling. Besides having high accuracy even for media without scale separation, the methods are mass conservative, which is an important feature for flow simulations.

\subsection{Model problem}

Darcy's law governs the fluid motion in the porous media. It states that the flux of a fluid at a certain location is proportional to the permeability of the location times the difference in fluid pressure across the location. Let $\boldsymbol{v}$ and $p$ respectively be the velocity and pressure of the fluid under consideration. Moreover, let $\kappa$ be the permeability of the domain $\Omega$. In general, $\kappa$ is a (possibly highly) heterogeneous tensor function. The partial differential equations describing the Darcy's flow is

$$
\boldsymbol{v}=-\kappa \nabla p .
$$

Part of the material in this section is reprinted from [24] with permission. 
Together with continuity equation and assuming the fluid is incompressible, we have the following system of partial differential equations

$$
\begin{aligned}
\kappa^{-1} \boldsymbol{v}+\nabla p=0 \quad & \text { in } \quad \Omega, \\
\operatorname{div}(\boldsymbol{v})=f \quad & \text { in } \quad \Omega .
\end{aligned}
$$

We close the system with no flux condition $\boldsymbol{v} \cdot \boldsymbol{n}=0$ on the boundary of the domain $\partial \Omega$, where $\boldsymbol{n}$ is the outward unit-normal vector on $\partial \Omega$.

In reservoir simulation, problem (2.1) needs to be solved multiple times, and therefore some efficient numerical methods are needed. There are in literature upscaling methods [30] and multiscale methods [1, 3, 37, 46, 50] that provide some efficient solution strategies for (2.1). For media with complex heterogeneities, these methods may not be able to produce accurate solutions. The goal of mixed GMsFEM to develop some enriched multiscale spaces which give accurate (velocity) solutions to (2.1) with few degrees of freedoms.

In the mixed GMsFEM considered in this section, we construct a enriched multiscale approximation space for the velocity field, $\boldsymbol{v}=-\kappa \nabla p$. For the pressure $p$, we will use piecewise constant approximations. As is in the discussion of mixed multiscale finite element methods, we will describe the mixed GMsFEM in a 2D setting. Again, the mixed GMsFEM for 3D problems should be analogous. For the notions of coarse and fine grids, coarse and fine edges, see Section 1.1. Let $Q_{H}$ be the space of piecewise constant functions with respect to the coarse grid $\mathscr{T}_{H}$. The approximation of the pressure $p$ will be obtained in this space, which is the same as 
the coarse pressure space in the mixed multiscale finite element methods. On the other hand, a set of multiscale basis functions for the velocity field $v$ are defined for each coarse edge $E_{i} \in \mathscr{E}_{H}$ and these basis functions are supported in the coarse neighborhood $\omega_{i}$ corresponding to the coarse edge $E_{i}$. Specifically, to obtain a basis function for a coarse edge $E_{i}$, we will solve a local problem in the coarse neighborhood $\omega_{i}$ with a given normal velocity on $E_{i}$ and zero normal velocity on the boundary $\partial \omega_{i}$. Notice that we can use multiple basis functions for each coarse edge $E_{i}$ by using various choices of normal velocity on $E_{i}$. Let $\left\{\boldsymbol{\psi}_{j}^{i}\right\}$ be the set of multiscale basis functions associated with the edge $E_{i}$. We define the multiscale space for the velocity field $v$ as the linear span of all local basis functions which is denoted as

$$
\boldsymbol{V}_{H}=\bigoplus_{E^{i} \in \mathscr{E}_{H}} \operatorname{span}\left\{\boldsymbol{\psi}_{j}^{i}\right\} .
$$

We also define $\boldsymbol{V}_{H}^{0}=\boldsymbol{V}_{H} \cap\left\{\boldsymbol{v} \in \boldsymbol{V}_{H}: \boldsymbol{v} \cdot \boldsymbol{n}=0\right.$ on $\left.\partial \Omega\right\}$ as a subspace of $\boldsymbol{V}_{H}$ consisting of vector fields with zero normal component on $\partial \Omega$; that is,

$$
\boldsymbol{V}_{H}^{0}=\bigoplus_{E^{i} \in \mathscr{E}_{H}^{0}} \operatorname{span}\left\{\boldsymbol{\psi}_{j}^{i}\right\}
$$

Given the above spaces, the mixed GMsFEM is to find $\left(\boldsymbol{v}_{H}, p_{H}\right) \in \boldsymbol{V}_{H}^{0} \times Q_{H}$ such that

$$
\begin{aligned}
\int_{\Omega} \kappa^{-1} \boldsymbol{v}_{H} \cdot \boldsymbol{w}_{H}-\int_{\Omega} \operatorname{div}\left(\boldsymbol{w}_{H}\right) p_{H} & =0, \quad \forall \boldsymbol{w}_{H} \in V_{H}^{0}, \\
\int_{\Omega} \operatorname{div}\left(\boldsymbol{v}_{H}\right) q_{H} & =\int_{\Omega} f q_{H}, \quad \forall q_{H} \in Q_{H} .
\end{aligned}
$$

In addition, we let $\boldsymbol{V}_{h} \times Q_{h}$ be the standard lowest-order Raviart-Thomas space and piecewise constant polynomials for the approximation of (2.1) on the fine grid 
$\mathscr{T}_{H}$. Then, the fine-grid solution $\left(\boldsymbol{v}_{h}, p_{h}\right)$ satisfies

$$
\begin{aligned}
\int_{\Omega} \boldsymbol{\kappa}^{-1} \boldsymbol{v}_{h} \cdot \boldsymbol{w}_{h}-\int_{\Omega} \operatorname{div}\left(\boldsymbol{w}_{h}\right) p_{h} & =0, \quad \forall \boldsymbol{w}_{h} \in \boldsymbol{V}_{h}^{0}, \\
\int_{\Omega} \operatorname{div}\left(\boldsymbol{v}_{h}\right) q_{h} & =\int_{\Omega} f q_{h}, \quad \forall q_{h} \in Q_{h},
\end{aligned}
$$

where $\boldsymbol{v}_{h} \cdot \boldsymbol{n}=g_{h}$ on $\partial D$ and $\boldsymbol{V}_{h}^{0}=\boldsymbol{V}_{h} \cap\left\{\boldsymbol{v} \in \boldsymbol{V}_{h}: \boldsymbol{v} \cdot \boldsymbol{n}=0\right.$ on $\left.\partial \Omega\right\}$. In terms of matrix representations, the above problem can be written as

$$
\begin{aligned}
M_{\text {fine }} \vec{V}_{h}+B_{\text {fine }}^{T} \vec{P}_{h} & =0 \\
B_{\text {fine }} \vec{V}_{h} & =\vec{F}_{h},
\end{aligned}
$$

where $\vec{V}_{h}$ and $\vec{P}_{h}$ are vectors of coefficients in the expansions of the solutions $\boldsymbol{v}_{h}$ and $p_{h}$ in the spaces $\boldsymbol{V}_{h}$ and $Q_{h}$, respectively. We remark that the fine-grid solution $\left(\boldsymbol{v}_{h}, p_{h}\right)$ is considered as a reference solution, and we will compare the accuracy of the multiscale solution $\left(\boldsymbol{v}_{H}, p_{H}\right)$ against the fine grid solution. Furthermore, it is easy to see that $Q_{H} \subset Q_{h}$. We will construct the multiscale space $\boldsymbol{V}_{H}$ so that $\boldsymbol{V}_{H} \subset \boldsymbol{V}_{h}$. Therefore, the mixed GMsFEM can be considered as a conforming method to approximate the fine-grid solution. In the next section, we will give the construction of the multiscale basis functions and the space $\boldsymbol{V}_{H}$.

2.2 The construction of multiscale basis functions

In this section, we will discuss the construction of the multiscale space $\boldsymbol{V}_{H}$ for the approximation of the velocity field. We will first introduce the snapshot space, which contains an extensive set of basis functions formed by solutions of local problems with all possible boundary conditions up to the fine-grid resolution. Then, we will present a space reduction technique which provides a systematic way to select 
the dominant modes in the snapshot space. This technique is based on a carefully designed local spectral problem giving a rapidly decaying residual. The resulting reduced space is obtained by the linear span of these dominant modes and is called the offline space. Notice that we use the terminology introduced in [33], where the notion of online space is also introduced. We emphasize that, since we consider problems without parameter dependence, the offline space is the same as the online space.

\subsubsection{Snapshot space}

In this section, we will define the snapshot space. Essentially, it is a space containing an extensive set of basis functions which are solutions of local problems with all possible boundary conditions up to the fine-grid resolution. Specifically, the functions in the snapshot space are $\kappa$-harmonic functions of unit-flux functions. In the following, we explain the detailed constructions. Let $E_{i} \in \mathscr{E}_{H}$ be a coarse edge. We will find $\left(\boldsymbol{\phi}_{j}^{i}, \xi_{j}^{i}\right)$ by solving the following problem on the coarse neighborhood $\omega_{i}$ corresponding to the edge $E_{i}$

$$
\begin{array}{r}
\kappa^{-1} \boldsymbol{\phi}_{j}^{i}+\nabla \xi_{j}^{i}=0 \quad \text { in } \quad \omega_{i}, \\
\operatorname{div}\left(\boldsymbol{\phi}_{j}^{i}\right)=\alpha_{j}^{i} \quad \text { in } \quad \omega_{i},
\end{array}
$$

subject to the boundary condition $\boldsymbol{\phi}_{j}^{i} \cdot \boldsymbol{n}_{i}=0$ on $\partial \omega_{i}$, where $\boldsymbol{n}_{i}$ denotes the outward unit-normal vector on $\partial \omega_{i}$, and $\alpha_{i}^{j}$ is constant on each coarse element. One key feature of our proposed approach is that the above problem (2.5) will be solved separately in the coarse-grid blocks forming $\omega_{i}$ (see Figure 1.1 for illustration). Therefore, 
we will need an extra boundary condition on $E_{i}$, which is discussed below. Notice that the coarse edge $E_{i}$ can be written as a union of fine-grid edges, namely, $E_{i}=\bigcup_{j=1}^{J_{i}} e_{j}$, where $J_{i}$ is the total number of fine-grid edges on $E_{i}$ and $e_{j}$ denotes a fine-grid edge. Let $\delta_{j}^{i}$ be a piecewise constant function defined on $E_{i}$ with respect to the fine-grid such that it has value 1 on $e_{j}$ and value 0 on the other fine-grid edges; that is,

$$
\delta_{j}^{i}=\left\{\begin{array}{ll}
1, & \text { on } e_{j}, \\
0, & \text { on other fine grid edges on } E_{i},
\end{array} \quad j=1,2, \cdots, J_{i} .\right.
$$

The remaining boundary condition on the coarse edge $E_{i}$ for the local problem $(2.5)$ is then taken as

$$
\boldsymbol{\phi}_{j}^{i} \cdot \boldsymbol{m}_{i}=\delta_{j}^{i} \quad \text { on } E_{j}
$$

where $\boldsymbol{m}_{i}$ is a fixed unit-normal vector on $E_{i}$. We remark that the constant $\alpha_{j}^{i}$ in (2.5) is chosen so that the compatibility condition $\int_{K_{l}} \alpha_{j}^{i}=\int_{E_{i}} \delta_{j}^{i}$ is satisfied, for all coarse element $K_{l} \subset \omega_{i}$. We also remark that, since $\boldsymbol{\phi}_{j}^{i} \cdot \boldsymbol{n}_{i}=0$ on the boundary of $\omega_{i}$, the vector field $\boldsymbol{\phi}_{j}^{i}$ can be extended to the rest of the domain $\Omega$ by defining $\boldsymbol{\phi}_{j}^{i}=0$ outside $\omega_{i}$. Furthermore, the above local problem (2.5) can be solved numerically on the underlying fine grid of $\omega_{i}$ by the lowest-order Raviart-Thomas element, so that the resulting velocity $\boldsymbol{\phi}_{j}^{i} \in \boldsymbol{V}_{h}$ (for simplicity, we keep the same notation for the discrete solution $\left.\boldsymbol{\phi}_{j}^{i}\right)$.

The collection of the solutions of the above local problems generates the snapshot space. We define the snapshot space $\boldsymbol{V}_{\text {snap }}$ by

$$
\boldsymbol{V}_{\text {snap }}=\operatorname{span}\left\{\boldsymbol{\phi}_{j}^{i}: \quad 1 \leq j \leq J_{i}, \quad 1 \leq i \leq N_{e}\right\}
$$


To simplify notation, we will use the following single-index notation

$$
\boldsymbol{V}_{\text {snap }}=\operatorname{span}\left\{\boldsymbol{\phi}_{i}: \quad 1 \leq i \leq M_{\text {snap }}\right\}
$$

where $M_{\text {snap }}=\sum_{i=1}^{N_{e}} J_{i}$ is the total number of snapshot basis functions.

Notice that each $\boldsymbol{\phi}_{i}$ is represented on the fine grid by the basis functions in $V_{h}$. Therefore, each $\boldsymbol{\phi}_{i}$ can be represented by a vector $\Phi_{i}$ containing the coefficients in the expansion of $\boldsymbol{\phi}_{i}$ in the fine-grid basis functions. Then, we define

$$
R_{\text {snap }}=\left[\Phi_{1}, \Phi_{2}, \ldots, \Phi_{M_{\text {snap }}}\right]
$$

which maps from the coarse space to the fine space.

\subsubsection{Offline space}

Following the general framework of [33], we will perform a space reduction on the snapshot space through the use of some local spectral problems. The reduced space is called the offline space. The purpose of this is to determine the important modes in the snapshot space and to obtain a smaller space for approximating the solution. In the general setting, we consider the spectral problem of finding a real number $\boldsymbol{\lambda}$ and a vector field $\boldsymbol{\psi} \in \boldsymbol{V}_{\text {snap }}$ such that

$$
a(\boldsymbol{\psi}, \boldsymbol{\phi})=\lambda s(\boldsymbol{\psi}, \boldsymbol{\phi}), \quad \forall \boldsymbol{\phi} \in \boldsymbol{V}_{\mathrm{snap}},
$$

where $a(\boldsymbol{\psi}, \boldsymbol{\phi})$ and $s(\boldsymbol{\psi}, \boldsymbol{\phi})$ are symmetric positive definite bilinear forms defined on $\boldsymbol{V}_{\text {snap }} \times \boldsymbol{V}_{\text {snap }}$. We consider $s(\boldsymbol{\psi}, \boldsymbol{\phi})$ as an inner product on $\boldsymbol{V}_{\text {snap }}$ and define a linear operator $\mathscr{A}: \boldsymbol{V}_{\text {snap }} \rightarrow \boldsymbol{V}_{\text {snap }}$ by

$$
s(\mathscr{A} \boldsymbol{\psi}, \boldsymbol{\phi})=a(\boldsymbol{\psi}, \boldsymbol{\phi})
$$


We assume that the operator $\mathscr{A}$ has rapidly decaying eigenvalues. Note that one can take $\mathscr{A}$ to be a compact operator.

In practice, solving the above global spectral problem (2.7) is inefficient. Therefore, the dimension reduction and the construction of the offline space are performed locally. In particular, the above spectral problem is solved for each coarse neighborhood $\omega_{i}$ corresponding to the coarse edge $E_{i}$. We let $\boldsymbol{V}_{\text {snap }}^{i}$ be the snapshot space associated with the coarse edge $E_{i}$, which is defined by

$$
V_{\text {snap }}^{i}=\operatorname{span}\left\{\boldsymbol{\phi}_{j}^{i}: \quad 1 \leq j \leq J_{i}\right\}
$$

The local spectral problem is: find a real number $\lambda>0$ and a function $\boldsymbol{\psi} \in \boldsymbol{V}_{\text {snap }}^{i}$ such that

$$
a_{i}(\boldsymbol{\psi}, \boldsymbol{\phi})=\lambda s_{i}(\boldsymbol{\psi}, \boldsymbol{\phi}), \quad \forall \boldsymbol{\phi} \in \boldsymbol{V}_{\text {snap }}^{i}
$$

We will consider two different choices of local spectral problems. One can possibly use oversampling ideas $[46,38,5,35]$ to achieve a better convergence rate (see Section $2.6)$.

Spectral problem 1: We take

$$
\begin{aligned}
& a_{i}(\boldsymbol{\psi}, \boldsymbol{\phi})=\int_{E_{i}} \kappa^{-1}\left(\boldsymbol{\psi} \cdot \boldsymbol{m}_{i}\right)\left(\boldsymbol{\phi} \cdot \boldsymbol{m}_{i}\right), \\
& s_{i}(\boldsymbol{\psi}, \boldsymbol{\phi})=\int_{\omega_{i}} \kappa^{-1} \boldsymbol{\psi} \cdot \boldsymbol{\phi}+\int_{\omega_{i}} \operatorname{div}(\boldsymbol{\psi}) \operatorname{div}(\boldsymbol{\phi}),
\end{aligned}
$$

where we recall that $\boldsymbol{m}_{i}$ is a fixed unit-normal on the coarse edge $E_{i}$.

Spectral problem 2: We take

$$
\begin{aligned}
& a_{i}(\boldsymbol{\psi}, \boldsymbol{\phi})=\int_{\omega_{i}} \kappa^{-1} \boldsymbol{\psi} \cdot \boldsymbol{\phi}, \\
& s_{i}(\boldsymbol{\psi}, \boldsymbol{\phi})=\int_{E_{i}}\left[p_{\boldsymbol{\psi}}\right]\left[p_{\boldsymbol{\phi}}\right],
\end{aligned}
$$


where $\left(\boldsymbol{\psi}, p_{\boldsymbol{\psi}}\right)$ and $\left(\boldsymbol{\phi}, p_{\boldsymbol{\phi}}\right)$ are solutions of the local problem $(2.5)$, and $[p]$ denotes the jump of the function $p$. Note that this spectral problem is related to the one used in [25].

In the following, we will focus our discussions on spectral problem 1. For spectral problem 2, we will only report its performance in Section 2.7 to show that it is also a promising way to obtain a reduced space.

Assume that the eigenvalues of (2.8) are arranged in increasing order

$$
\lambda_{1}^{i}<\lambda_{2}^{i}<\cdots<\lambda_{J_{i}}^{i}
$$

where $\lambda_{k}^{i}$ denotes the $k$-th eigenvalue for the coarse neighborhood $\omega_{i}$. The corresponding eigenvectors are denoted by $Z_{k}^{i}=\left(Z_{k j}^{i}\right)_{j=1}^{J_{i}}$, where $Z_{k j}^{i}$ is the $j$-th component of the vector $Z_{k}^{i}$. We will use the first $l_{i}$ eigenfunctions to form the offline space. We remark that we assume the eigenvalues are strictly increasing (here, we refer to the inverse of $\mathscr{A}$, cf. (2.7)) only to simplify the discussion. In practice, if there are multiple eigenvectors corresponding to a specific eigenvalue, then we will take all these eigenvectors to be part of the basis functions when the corresponding eigenvalue is selected. Using the eigenfunctions, offline basis functions can be constructed as

$$
\boldsymbol{\psi}_{k}^{i}=\sum_{j=1}^{J_{i}} Z_{k j}^{i} \boldsymbol{\phi}_{j}^{i}, \quad k=1,2, \cdots, l_{i} .
$$

The global offline space is then

$$
V_{\mathrm{off}}=\operatorname{span}\left\{\boldsymbol{\psi}_{k}^{i}: \quad 1 \leq k \leq l_{i}, \quad 1 \leq i \leq N_{e}\right\}
$$

As an illustration of how the multiscale basis functions look like, we revisit the 


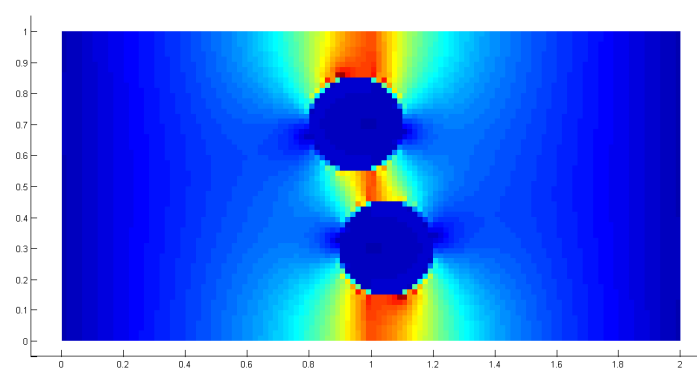

(a) Magnitude of $\boldsymbol{\psi}_{1}^{i}$

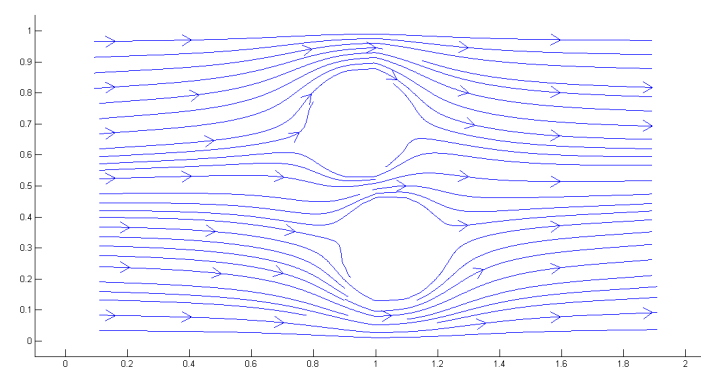

(b) Streamline of $\boldsymbol{\psi}_{1}^{i}$

Figure 2.1: First multiscale basis function $\boldsymbol{\psi}_{1}^{i}$ by mixed GMsFEM for the local coefficient in Figure 1.3(a).

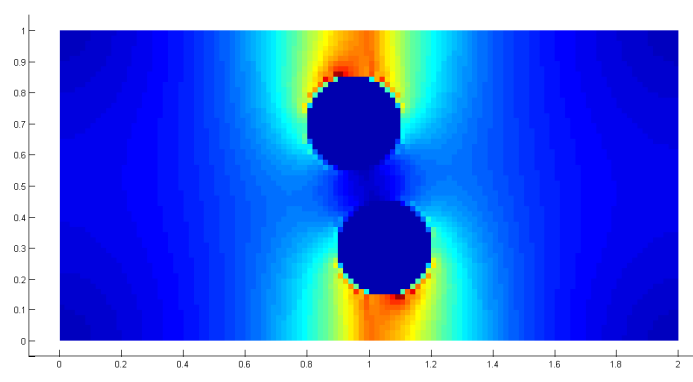

(a) Magnitude of $\boldsymbol{\psi}_{2}^{i}$

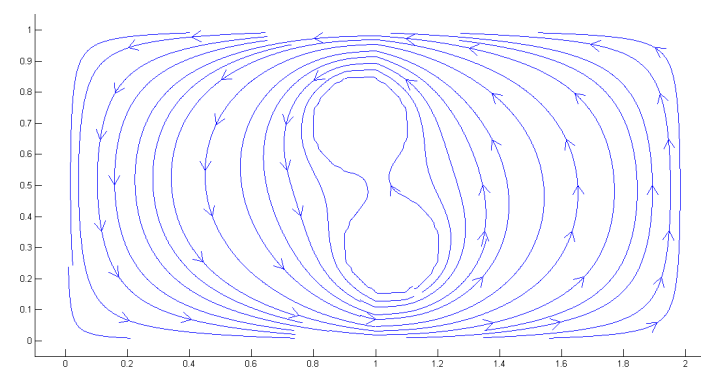

(b) Streamline of $\boldsymbol{\psi}_{2}^{i}$

Figure 2.2: Second multiscale basis function $\boldsymbol{\psi}_{2}^{i}$ by mixed GMsFEM for the local coefficient in Figure 1.3(a).

example local coefficient in Figure 1.3(a) in which basis function of mixed MsFEM cannot capture the local physics. For this local coefficient, the first few basis functions constructed by mixed GMsFEM are shown in Figures 2.1 - 2.3. These functions all avoid going into the blue regions, so we expect them to provide better representations of the global solution than the mixed MsFEM basis function shown in Figure 1.3.

To simplify notation, we will use the following single-index notation

$$
\boldsymbol{V}_{\mathrm{off}}=\operatorname{span}\left\{\boldsymbol{\psi}_{k}: \quad 1 \leq k \leq M_{\mathrm{off}}\right\}
$$




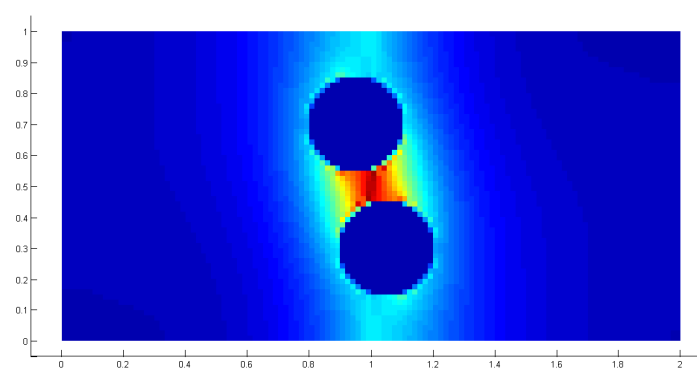

(a) Magnitude of $\boldsymbol{\psi}_{3}^{i}$

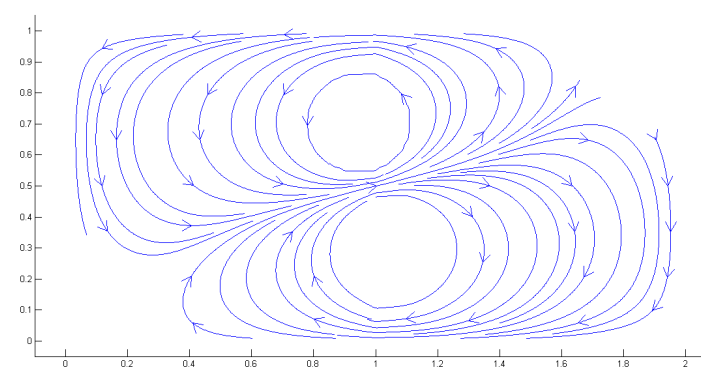

(b) Streamline of $\boldsymbol{\psi}_{3}^{i}$

Figure 2.3: Third multiscale basis function $\boldsymbol{\psi}_{3}^{i}$ by mixed GMsFEM for the local coefficient in Figure 1.3(a).

where $M_{\mathrm{off}}=\sum_{i=1}^{N_{e}} l_{i}$ is the total number of offline basis functions. This space will be used as the approximation space for the velocity; that is, $\boldsymbol{V}_{H}=\boldsymbol{V}_{\text {off }}$ in the GMsFEM system (2.2). Furthermore, we define $\boldsymbol{V}_{\text {off }}^{0}$ as the restriction of $\boldsymbol{V}_{\text {off }}$ formed by the linear span of all basis functions $\boldsymbol{\psi}_{k}$ corresponding to interior coarse edges only. Thus, all vectors in $\boldsymbol{V}_{\text {off }}^{0}$ have zero normal component on the global domain boundary $\partial \Omega$. In term of matrix representations, the above eigenvalue problem (2.8) can be expressed as

$$
A_{\mathrm{snap}}^{i} Z_{k}^{i}=\lambda_{k}^{i} S_{\mathrm{snap}}^{i} Z_{k}^{i}
$$

where

$$
\begin{gathered}
A_{\text {snap }}^{i}=\left[\left(A_{\mathrm{snap}}^{i}\right)_{m n}\right]=a_{i}\left(\boldsymbol{\phi}_{m}^{i}, \boldsymbol{\phi}_{n}^{i}\right)=R_{\mathrm{snap}}^{T} A_{\text {fine }}^{i} R_{\text {snap }} \\
\text { and } \\
S_{\text {snap }}^{i}=\left[\left(S_{\text {snap }}^{i}\right)_{m n}\right]=s_{i}\left(\boldsymbol{\phi}_{m}^{i}, \boldsymbol{\phi}_{n}^{i}\right)=R_{\text {snap }}^{T} S_{\text {fine }}^{i} R_{\text {snap }} .
\end{gathered}
$$

We note that $A_{\text {fine }}^{i}$ and $S_{\text {fine }}^{i}$ denote analogous fine-scale matrices that use fine-grid 
basis functions. Notice that each $\boldsymbol{\psi}_{k}$ is represented on the fine grid. Therefore, each $\boldsymbol{\psi}_{k}$ can be represented by a vector $\Psi_{k}$ containing the coefficients in the expansion of $\boldsymbol{\psi}_{k}$ in the fine-grid basis functions. Then, we define

$$
R_{\text {off }}=\left[\Psi_{1}, \Psi_{2}, \ldots, \Psi_{M_{\text {off }}}\right]
$$

which maps from the offline space to the fine space. Similar to (2.4), the GMsFEM system (2.2) can be represented in matrix form as follows.

$$
\begin{aligned}
R_{\text {off }}^{T} M_{\text {fine }} R_{\text {off }} \vec{V}_{H}+R_{\text {off }}^{T} B_{\text {fine }}^{T} G_{H} \vec{P}_{H} & =0 \\
G_{H}^{T} B_{\text {fine }} R_{\text {off }} \vec{V}_{H} & =G_{H}^{T} \vec{F}_{h},
\end{aligned}
$$

where $G_{H}$ is the restriction operator from $Q_{H}$ into $Q_{h}$, and $\vec{V}_{H}$ and $\vec{P}_{H}$ are vectors of coefficients in the expansions of the solutions $\boldsymbol{v}_{H}$ and $p_{H}$ in the spaces $\boldsymbol{V}_{H}$ and $Q_{H}$, respectively. From (2.13), it is easy to see that implementing the mixed GMsFEM requires the construction of the fine-grid matrices $M_{\text {fine }}$ and $B_{\text {fine }}$ as well as the offline matrix $R_{\text {off }}$.

To contrast the performance of mixed GMsFEM and mixed MsFEM, we go back to the example in Section 1 where mixed MsFEM fails to produce a reliable approximation, see Figure 1.5. If the same fine problem is coarsened by mixed GMsFEM, the velocity solutions are depicted in Figure 2.4. It is obvious that the coarse velocity approximations by mixed GMsFEM are much better than mixed MsFEM. In fact, the coarse velocity approximations by mixed GMsFEM look almost identical to the reference fine grid solution for this example. 


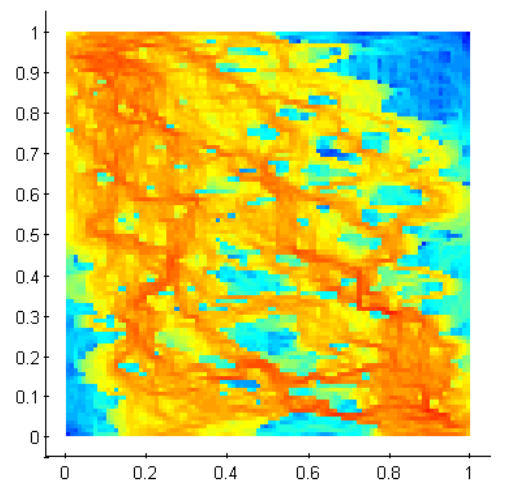

(a) Reference sol'n (dim=29799)

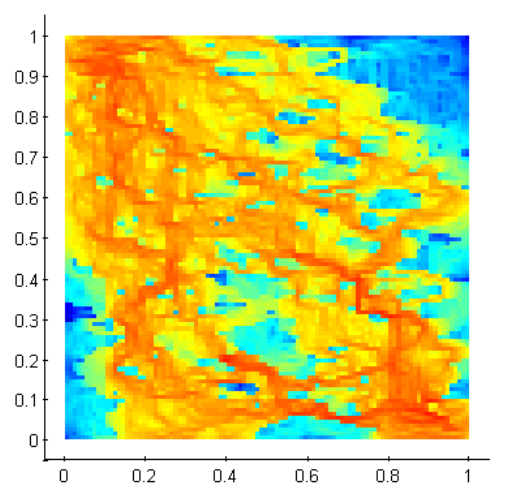

(c) Mixed GMsFEM sol'n (dim=279)

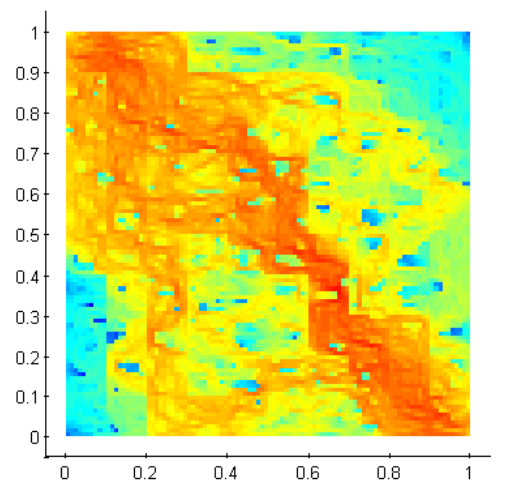

(b) Mixed MsFEM sol'n $(\operatorname{dim}=279)$

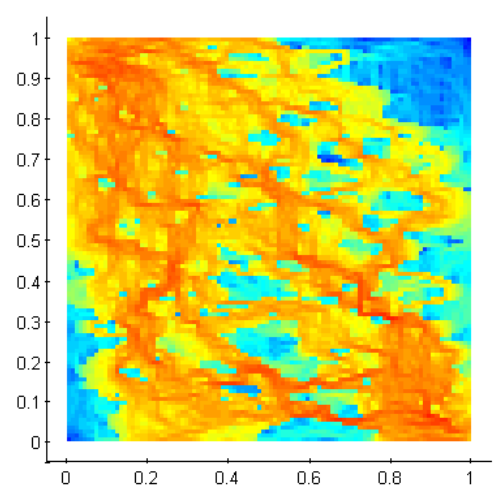

(d) Mixed GMsFEM sol'n (dim=639)

Figure 2.4: Fine and coarse scale solutions when $\kappa=\kappa_{\text {channel }}$.

\subsection{Optimization viewpoint of the basis functions}

In this section, we present an optimization viewpoint for the basis functions obtained by the local spectral problem (2.8). Recall that, for each coarse neighborhood $\omega_{i}$, we will solve the spectral problem (2.8) to get a sequence of eigenpairs $\left(\lambda_{k}^{i}, Z_{k}^{i}\right)$. We will show, by means of an optimization approach, that the eigenfunction $Z_{k}^{i}$ is furthest away from the space spanned by the previous eigenvectors $Z_{1}^{i}, \cdots, Z_{k-1}^{i}$. Thus, whenever a new basis function is added, this basis function will represent an 
important component in the solution space.

Assume that $k-1$ basis functions, $\boldsymbol{\psi}_{1}, \cdots, \boldsymbol{\psi}_{k-1}$, are selected for a specific coarse neighborhood $\omega_{i}$. Let $W$ be the space spanned by these functions. To find an additional basis function, we will find a function $\boldsymbol{\psi}_{k}$ orthogonal to the space $W$ and furthest away from the space $W$. To be more specific, we let $W^{\perp}$ be the orthogonal complement of $W$ with respect to the inner product defined by the bilinear form $s_{i}(\boldsymbol{\psi}, \boldsymbol{\phi})$; namely,

$$
W^{\perp}=\left\{\boldsymbol{v} \in \boldsymbol{V}_{\text {snap }}^{i} \mid s_{i}(\boldsymbol{v}, \boldsymbol{\psi})=0, \forall \boldsymbol{\psi} \in W\right\}
$$

Then, the function $\boldsymbol{\psi}_{k}$ is obtained by the following constrained optimization problem

$$
\begin{aligned}
& \boldsymbol{\psi}_{k}=\arg \max _{\boldsymbol{\psi} \in W^{\perp}} s_{i}(\boldsymbol{\psi}-w, \boldsymbol{\psi}-w), \\
& \text { subject to } a_{i}(\boldsymbol{\psi}, \boldsymbol{\psi})=1
\end{aligned}
$$

for all $w \in W$. By orthogonality, the above problem can be formulated as

$$
\begin{gathered}
\boldsymbol{\psi}_{k}=\arg \max _{\boldsymbol{\psi} \in W^{\perp}} s_{i}(\boldsymbol{\psi}, \boldsymbol{\psi}), \\
\text { subject to } a_{i}(\boldsymbol{\psi}, \boldsymbol{\psi})=1 .
\end{gathered}
$$

It is well-known that the Euler-Lagrange equation for the above optimization problem is

$$
\begin{aligned}
& s_{i}\left(\boldsymbol{\psi}_{k}, \boldsymbol{\psi}\right)-\mu a_{i}\left(\boldsymbol{\psi}_{k}, \boldsymbol{\psi}\right)=0, \quad \forall \boldsymbol{\psi} \in W^{\perp}, \\
& a_{i}\left(\boldsymbol{\Psi}_{k}, \boldsymbol{\Psi}_{k}\right)=1
\end{aligned}
$$

where $\mu$ is the Lagrange multiplier. The above condition explains why we select the eigenfunctions of the spectral problem (2.8) as basis functions. 


\subsection{Postprocessing}

In this section, we present a postprocessing technique to enhance the conservation property of the mixed GMsFEM solution. First, notice that the mixed GMsFEM is conservative on the coarse-grid level. Specifically, the solution of (2.2) satisfies

$$
\int_{\partial K} \boldsymbol{v}_{H} \cdot \boldsymbol{n}=\int_{K} f
$$

for every coarse-grid block $K$. This is a direct consequence of the second equation of (2.2) and the fact that $Q_{H}$ contains functions that are constant in each coarse block. When $f$ has fine-scale oscillation in some coarse blocks, the velocity field needs to be postprocessed in these coarse blocks. In porous media applications, there are only a few coarse blocks where the sources and sinks are. In the following, we will construct a postprocessed velocity $\boldsymbol{v}_{h}^{\star}$ such that conservation on the fine grid is obtained, that is,

$$
\int_{\partial \tau}\left(\boldsymbol{v}_{h}^{\star} \cdot \boldsymbol{n}\right)=\int_{\tau} f, \quad \forall \tau \in \mathscr{T}_{h}
$$

In particular, for each coarse-grid block $K$, we find $\left(v_{h}^{\star}, p_{h}^{\star}\right) \in V_{h}(K) \times Q_{h}(K)$ such that $\boldsymbol{v}_{h}^{\star} \cdot \boldsymbol{n}=\boldsymbol{v}_{H} \cdot \boldsymbol{n}$ and

$$
\begin{aligned}
\int_{K} \kappa^{-1} \boldsymbol{v}_{h}^{\star} \cdot \boldsymbol{w}_{h}-\int_{K} p_{h}^{\star} \operatorname{div}\left(\boldsymbol{w}_{h}\right) & =0, \quad \forall \boldsymbol{w}_{h} \in \boldsymbol{V}_{h}^{0}(K) \\
\int_{K} \operatorname{div}\left(\boldsymbol{v}_{h}^{\star}\right) q_{h} & =\int_{K} f q_{h}, \quad \forall q_{h} \in Q_{h}(K) .
\end{aligned}
$$

In the single-phase and two-phase flow and transport simulation experiments below, we will apply this postprocessing technique to obtain conservative velocity fields on the fine-grid level. We remark that this postprocessing is only needed in the 
coarse blocks where the source term $f$ is non-constant. Therefore, computing the postprocessed velocity $\boldsymbol{v}_{h}^{\star}$ is very efficient.

\subsection{Convergence of the mixed GMsFEM}

In this section, we will prove the convergence of the mixed GMsFEM (2.2). The analysis consists of two main steps. In the first step, we will construct a projection of the fine-grid velocity field $v_{h}$ to the snapshot space, and derive an error estimate for such projection. In the second step, we will derive an estimate for the difference between the projection of the fine-grid velocity and the GMsFEM solution. Combining the above two steps, we obtain an estimate for the difference between the fine-grid and the GMsFEM solution.

Recall that $\left(\boldsymbol{v}_{h}, p_{h}\right) \in \boldsymbol{V}_{h} \times Q_{h}$ is the fine-grid solution obtained in (2.3). We will define a projection $\widehat{\boldsymbol{v}} \in V_{\text {snap }}$ as follows. Let $K$ be a coarse-grid block and let $\bar{f}=\frac{1}{|K|} \int_{K} f$ be the average value of $f$ over $K$. Then, the restriction of $\widehat{\boldsymbol{v}}$ on $K$ is obtained by solving the following problem

$$
\begin{aligned}
\kappa^{-1} \widehat{\boldsymbol{v}}+\nabla \widehat{p} & =0 \quad \text { in } \quad K, \\
\operatorname{div}(\widehat{\boldsymbol{v}}) & =\bar{f} \quad \text { in } \quad K,
\end{aligned}
$$

subject to the following conditions

$$
\widehat{\boldsymbol{v}} \cdot \boldsymbol{n}=\boldsymbol{v}_{h} \cdot \boldsymbol{n}, \text { on } \partial K \text { and } \int_{K} \widehat{p}=\int_{K} p_{h} .
$$

We remark that the above problem (2.17)-(2.18) is solved on the fine grid, and therefore we have $\widehat{\boldsymbol{v}} \in \boldsymbol{V}_{h}$. By the construction, we also have $\widehat{\boldsymbol{v}} \in \boldsymbol{V}_{\text {snap }}$. 
Now, we introduce some notations for the following analysis. Let $D$ be an open set. For a scalar function $q \in L^{2}(D)$, the $L^{2}$ norm is $\|q\|_{L^{2}(D)}^{2}=\int_{D} q^{2}$; and for a vector field $\boldsymbol{v}$, we define the weighted $L^{2}$ norm $\|\boldsymbol{v}\|_{\mathcal{K}^{-1}, D}^{2}=\int_{D} \kappa^{-1}|\boldsymbol{v}|^{2}$. Moreover, the notation $H\left(\operatorname{div} ; D ; \kappa^{-1}\right)$ denotes the Sobolev space containing vector fields $\boldsymbol{v}$ with

$$
\|\boldsymbol{v}\|_{H(\operatorname{div} ; D) ; K^{-1}}<\infty
$$

where the norm $\|\boldsymbol{v}\|_{H(\operatorname{div} ; D) ; \kappa^{-1}}^{2}:=\|\boldsymbol{v}\|_{\mathcal{K}^{-1}, D}+\|\operatorname{div}(\boldsymbol{v})\|_{L^{2}(D)}^{2}$. If $\kappa=1$, we write $H(\operatorname{div} ; D)=H\left(\operatorname{div} ; D ; \kappa^{-1}\right)$. Furthermore, $\alpha \preceq \beta$ means that there is a uniform constant $C>0$ such that the two quantities $\alpha$ and $\beta$ satisfy $\alpha \leq C \beta$.

Next, we prove the following estimate for $\widehat{\boldsymbol{v}}$.

Lemma 2.5.1 Let $\left(\boldsymbol{v}_{h}, p_{h}\right) \in \boldsymbol{V}_{h} \times Q_{h}$ be the fine-grid solution obtained in (2.3) and $\widehat{\boldsymbol{v}} \in \boldsymbol{V}_{h} \cap \boldsymbol{V}_{\text {snap }}$ be the solution of (2.17)-(2.18). We have

$$
\int_{\Omega} \kappa^{-1}\left|\boldsymbol{v}_{h}-\widehat{\boldsymbol{v}}\right|^{2} \preceq \kappa_{\min }^{-1} \sum_{i=1}^{N_{e}}\|f-\bar{f}\|_{L^{2}\left(K_{i}\right)}^{2}
$$

where $\kappa_{\min }$ is the minimum of $\kappa$ over $\Omega$

Proof. Let $K \in \mathscr{T}_{H}$ be a given coarse-grid block. First, substracting (2.3) by the variational form of $(2.17)$, we have

$$
\begin{array}{r}
\int_{K} \boldsymbol{\kappa}^{-1}\left(\boldsymbol{v}_{h}-\widehat{\boldsymbol{v}}\right) \cdot \boldsymbol{w}_{h}-\int_{K} \operatorname{div}\left(\boldsymbol{w}_{h}\right)\left(p_{h}-\widehat{p}\right)=0, \quad \forall \boldsymbol{w}_{h} \in V_{h}^{0}(K), \\
\int_{K} \operatorname{div}\left(\boldsymbol{v}_{h}-\widehat{\boldsymbol{v}}\right) q_{h}=\int_{K}(f-\bar{f}) q_{h}, \quad \forall q_{h} \in Q_{h}(K),
\end{array}
$$

where $Q_{h}(K)$ is the restriction of $Q_{h}$ on $K$ and $\boldsymbol{V}_{h}^{0}(K)$ is the restriction of $\boldsymbol{V}_{h}$ on $K$ containing vector fields with zero normal component on $\partial K$. Taking $\boldsymbol{w}_{h}=\boldsymbol{v}_{h}-\widehat{\boldsymbol{v}}$ and 
$q_{h}=p_{h}-\widehat{p}$ in $(2.20)$, and summing up the resulting equations, we have

$$
\int_{K} \kappa^{-1}\left(\boldsymbol{v}_{h}-\widehat{\boldsymbol{v}}\right) \cdot\left(\boldsymbol{v}_{h}-\widehat{\boldsymbol{v}}\right)=\int_{K}(f-\bar{f})\left(p_{h}-\widehat{p}\right) .
$$

Recall that the Raviart-Thomas element satisfies the following inf-sup condition [12]:

$$
\left\|q_{h}\right\|_{L^{2}(K)} \preceq \sup _{\boldsymbol{w}_{h} \in \boldsymbol{V}_{h}^{0}(K)} \frac{\int_{K} \operatorname{div}\left(\boldsymbol{w}_{h}\right) q_{h}}{\left\|\boldsymbol{w}_{h}\right\|_{H(\operatorname{div} ; K)}}, \quad \forall q_{h} \in Q_{h}(K) \cap L_{0}^{2}(K) .
$$

Using the inf-sup condition (2.22) and the error equation (2.20), we have

$$
\left\|p_{h}-\widehat{p}\right\|_{L^{2}(K)} \preceq \kappa_{\min , K}^{-\frac{1}{2}}\left\|\boldsymbol{v}_{h}-\widehat{\boldsymbol{v}}\right\|_{\kappa^{-1}, K},
$$

where $\kappa_{\min , K}$ is the minimum of $\kappa$ over the coarse element $K$. Finally, by $(2.21)$, we obtain

$$
\left\|\boldsymbol{v}_{h}-\widehat{\boldsymbol{v}}\right\|_{\kappa^{-1}, K} \preceq \kappa_{\min , K}^{-\frac{1}{2}}\|f-\bar{f}\|_{L^{2}(K)} .
$$

Collecting results for all coarse-grid blocks, we obtain the desired estimate (2.19).

To simplify the notations, we will consider the case with homogeneous Neumann boundary condition in (2.1). In this case, the multiscale basis functions are obtained only for interior coarse edges. We emphasize that the same analysis can be applied to the non-homogeneous case. Let $N_{0}$ be the number of interior coarse edges. For each interior coarse edge $E_{i}$, we assume that there exists a basis function $\boldsymbol{\psi}_{r_{i}}^{i} \in \boldsymbol{V}_{\text {off }}^{0}$, $1 \leq r \leq l_{i}$, such that $\int_{E_{i}} \boldsymbol{\psi}_{r_{i}}^{i} \cdot \boldsymbol{m}_{i} \neq 0$. We remark that this is a reasonable assumption otherwise all basis functions are divergence free. As a key step in the proof of the main result in Theorem 2.5.3, we first prove the following inf-sup condition.

Theorem 2.5.2 For all $p \in Q_{H}$, we have

$$
\|p\|_{L^{2}(D)} \preceq C_{\text {infsup }} \sup _{\boldsymbol{w} \in \boldsymbol{V}_{\mathrm{off}}^{0}} \frac{\int_{\Omega} \operatorname{div}(\boldsymbol{w}) p}{\|\boldsymbol{w}\|_{H\left(\operatorname{div} ; \Omega ; \kappa^{-1}\right)}},
$$


where $C_{\text {infsup }}=\left(\max _{1 \leq i \leq N_{0}} \min _{r} \int_{\omega_{i}} \kappa^{-1} \boldsymbol{\psi}_{r}^{i} \cdot \boldsymbol{\psi}_{r}^{i}+1\right)^{\frac{1}{2}}$ and the minimum is taken over all indices $r$ with the property $\int_{E_{i}} \boldsymbol{\psi}_{r}^{i} \cdot \boldsymbol{m}_{i} \neq 0$.

Proof. Let $p \in Q_{H}$. We consider the following Neumann problem

$$
\begin{aligned}
& \Delta \zeta=p, \quad \text { in } D, \\
& \frac{\partial \zeta}{\partial n}=0, \quad \text { on } \partial D .
\end{aligned}
$$

We assume that the solution $\zeta \in H^{2}(D)$ and we let $\boldsymbol{\eta}=\nabla \zeta$. Then we will define $\boldsymbol{w} \in \boldsymbol{V}_{\text {off }}^{0}$ so that $\operatorname{div}(\boldsymbol{w})=p$ in $D$. Specifically, the function $\boldsymbol{w}$ is defined in the following way

$$
\boldsymbol{w}=\sum_{i=1}^{N_{0}} w_{i} \boldsymbol{\psi}_{r_{i}}^{i}, \quad w_{i}=\int_{E_{i}} \boldsymbol{\eta} \cdot \boldsymbol{m}_{i}
$$

and, in this proof only, we normalize the basis functions so that $\int_{E_{i}} \boldsymbol{\psi}_{r_{i}}^{i} \cdot \boldsymbol{m}_{i}=1$. Thus,

$$
\int_{\Omega} p^{2}=\int_{\Omega} \operatorname{div}(\boldsymbol{\eta}) p=\sum_{i=1}^{N_{0}} \int_{E_{i}}\left(\boldsymbol{\eta} \cdot \boldsymbol{m}_{i}\right)[p]=\sum_{i=1}^{N_{0}} \int_{E_{i}} w_{i}\left(\boldsymbol{\psi}_{r_{i}}^{i} \cdot \boldsymbol{m}_{i}\right)[p]=\int_{\Omega} \operatorname{div}(\boldsymbol{w}) p,
$$

where $[p]$ is the jump of $p$ across the coarse edge.

To show (2.23), it remains to estimate $\|\boldsymbol{w}\|_{\boldsymbol{\kappa}^{-1}, \Omega}$. Notice that,

$$
\|\boldsymbol{w}\|_{\kappa^{-1}, \Omega}^{2}=\int_{\Omega} \kappa^{-1} \boldsymbol{w} \cdot \boldsymbol{w} \leq \sum_{i=1}^{N_{0}} \int_{\omega_{i}} \kappa^{-1} w_{i}^{2} \boldsymbol{\psi}_{r_{i}}^{i} \cdot \boldsymbol{\psi}_{r_{i}}^{i}
$$

For each $i$, we have $w_{i}^{2} \leq H \int_{E_{i}}\left(\boldsymbol{\eta} \cdot \boldsymbol{m}_{i}\right)^{2}$. Thus,

$$
\|\boldsymbol{w}\|_{\mathcal{K}^{-1}, \Omega}^{2} \preceq H\left(\max _{1 \leq i \leq N_{0}} \int_{\omega_{i}} \boldsymbol{\kappa}^{-1} \boldsymbol{\psi}_{r_{i}}^{i} \cdot \boldsymbol{\psi}_{r_{i}}^{i}\right) \sum_{K \in \mathscr{T}_{H}} \int_{\partial K}(\boldsymbol{\eta} \cdot \boldsymbol{n})^{2} .
$$

Since the above inequality holds for any $\boldsymbol{\psi}_{r}^{i}$ such that $\int_{E_{i}} \boldsymbol{\psi}_{r}^{i} \cdot \boldsymbol{m}_{i} \neq 0$, we have

$$
\|\boldsymbol{w}\|_{\mathcal{K}^{-1}, \Omega}^{2} \preceq H\left(\max _{1 \leq i \leq N_{0}} \min _{r} \int_{\omega_{i}} \kappa^{-1} \boldsymbol{\psi}_{r}^{i} \cdot \boldsymbol{\psi}_{r}^{i}\right) \sum_{K \in \mathscr{T}_{H}} \int_{\partial K}(\boldsymbol{\eta} \cdot \boldsymbol{n})^{2},
$$

where the above minimum is taken over all indices $r$ with the property $\int_{E_{i}} \boldsymbol{\Psi}_{r}^{i} \cdot \boldsymbol{m}_{i} \neq 0$.

Finally, we will estimate $\int_{\partial K}(\boldsymbol{\eta} \cdot \boldsymbol{n})^{2}$ for every coarse grid block $K$. By the 
Green's identity, we have

$$
\int_{\partial K}(\boldsymbol{\eta} \cdot \boldsymbol{n}) z=\int_{K} \nabla \zeta \cdot \nabla \tilde{z}+\int_{K} p \tilde{z}
$$

where $z \in H^{\frac{1}{2}}(\partial K)$ and $\tilde{z} \in H^{1}(K)$ is any extension of $z$ in $K$. By Cauchy-Schwarz inequality,

$$
\begin{aligned}
\int_{\partial K}(\eta \cdot n) z & =\int_{K} \nabla \zeta \cdot \nabla \tilde{z}+\int_{K} p \tilde{z} \\
& \preceq\left(\|\nabla \zeta\|_{L^{2}(K)}^{2}+\|p\|_{L^{2}(K)}^{2}\right)^{\frac{1}{2}}\|\tilde{z}\|_{H^{1}(K)} \\
& \leq C_{K}\left(\|\nabla \zeta\|_{L^{2}(K)}^{2}+\|p\|_{L^{2}(K)}^{2}\right)^{\frac{1}{2}}\|z\|_{H^{\frac{1}{2}}(\partial K)},
\end{aligned}
$$

where the constant $C_{K}$ depends on $K$. Thus,

$$
\int_{\partial K}(\boldsymbol{\eta} \cdot \boldsymbol{n})^{2} \leq C_{K}^{2}\left(\|\nabla \zeta\|_{L^{2}(K)}^{2}+\|p\|_{L^{2}(K)}^{2}\right) .
$$

By a scaling argument, we obtain

$$
H \int_{\partial K}(\boldsymbol{\eta} \cdot \boldsymbol{n})^{2} \preceq\|\nabla \zeta\|_{L^{2}(K)}^{2}+\|p\|_{L^{2}(K)}^{2} .
$$

Summing the above over all coarse grid blocks $K$ and using $\|\nabla \zeta\|_{L^{2}(K)}^{2} \preceq\|p\|_{L^{2}(K)}^{2}$, we have $H \sum_{K \in \mathscr{T}_{H}} \int_{\partial K}(\boldsymbol{\eta} \cdot \boldsymbol{n})^{2} \preceq\|p\|_{L^{2}(K)}^{2}$. Hence, we obtain the desired bound (2.23) by using (2.24) and (2.25).

Now we state and prove the convergence theorem for the mixed GMsFEM for Darcy's flow (2.2).

Theorem 2.5.3 Let $\boldsymbol{v}_{h}$ be the fine-grid solution obtained in (2.3) and $\boldsymbol{v}_{H}$ be the mixed GMsFEM solution obtained in (2.2). Then, the following estimate holds

$$
\int_{\Omega} \kappa^{-1}\left|\boldsymbol{v}_{h}-\boldsymbol{v}_{H}\right|^{2} \preceq C_{\mathrm{infsup}}^{2} \Lambda^{-1} \sum_{i=1}^{N_{0}} a_{i}(\widehat{\boldsymbol{v}}, \widehat{\boldsymbol{v}})+\kappa_{\min }^{-1} \sum_{K \in \mathscr{T}_{H}}\|f-\bar{f}\|_{L^{2}(K)}^{2},
$$


where $\Lambda=\min _{1 \leq i \leq N_{0}} \lambda_{l_{i}+1}^{(i)}$ and $\widehat{\boldsymbol{v}}$ is the projection of $\boldsymbol{v}_{h}$ defined in (2.17)-(2.18).

Proof. Subtracting (2.3) by (2.2), and using the fact that $\boldsymbol{V}_{\text {off }}^{0} \subset \boldsymbol{V}_{h}^{0}$ and $Q_{H} \subset$ $Q_{h}$, we have

$$
\begin{aligned}
& \int_{\Omega} \boldsymbol{\kappa}^{-1}\left(\boldsymbol{v}_{h}-\boldsymbol{v}_{H}\right) \cdot \boldsymbol{w}_{H}-\int_{\Omega} \operatorname{div}\left(\boldsymbol{w}_{H}\right)\left(p_{h}-p_{H}\right)=0, \quad \forall \boldsymbol{w}_{H} \in \boldsymbol{V}_{\mathrm{off}}^{0}, \\
& \int_{\Omega} \operatorname{div}\left(\boldsymbol{v}_{h}-\boldsymbol{v}_{H}\right) q_{H}=0, \quad \forall q_{H} \in Q_{H} .
\end{aligned}
$$

By (2.20), for each coarse-grid block $K$, we have

$$
\int_{K} \operatorname{div}\left(\boldsymbol{v}_{h}-\widehat{\boldsymbol{v}}\right) q_{H}=\int_{K}(f-\bar{f}) q_{H}=0, \quad \forall q_{H} \in Q_{H}
$$

since $q_{H}$ is a constant function on $K$. Similarly, since $\operatorname{div}\left(\boldsymbol{w}_{H}\right)$ is a constant function for any $\boldsymbol{w}_{H} \in \boldsymbol{V}_{\text {off }}^{0}$, by $(2.18)$, we have

$$
\int_{\Omega} \operatorname{div}\left(\boldsymbol{w}_{H}\right) p_{h}=\int_{\Omega} \operatorname{div}\left(\boldsymbol{w}_{H}\right) \widehat{p}
$$

Thus, (2.27) can be written as

$$
\begin{aligned}
\int_{\Omega} \boldsymbol{\kappa}^{-1}\left(\boldsymbol{v}_{h}-\boldsymbol{v}_{H}\right) \cdot \boldsymbol{w}_{H}-\int_{\Omega} \operatorname{div}\left(\boldsymbol{w}_{H}\right)\left(\widehat{p}-p_{H}\right) & =0, \quad \forall \boldsymbol{w}_{H} \in \boldsymbol{V}_{\mathrm{off}}^{0}, \\
\int_{\Omega} \operatorname{div}\left(\widehat{\boldsymbol{v}}-\boldsymbol{v}_{H}\right) q_{H} & =0, \quad \forall q_{H} \in Q_{H} .
\end{aligned}
$$

Notice that $\widehat{\boldsymbol{v}} \in \boldsymbol{V}_{\text {snap. }}$. We can therefore write $\widehat{\boldsymbol{v}}$ as

$$
\widehat{\boldsymbol{v}}=\sum_{i=1}^{N_{0}} \sum_{k=1}^{J_{i}} \widehat{v}_{i k} \boldsymbol{\psi}_{k}^{i} .
$$

We then define $\widehat{\boldsymbol{v}}_{\text {off }} \in \boldsymbol{V}_{\text {off }}$ by

$$
\widehat{\boldsymbol{v}}_{\mathrm{off}}=\sum_{i=1}^{N_{0}} \sum_{k=1}^{l_{i}} \widehat{v}_{i k} \boldsymbol{\Psi}_{k}^{i}
$$

where we recall that $l_{i} \leq J_{i}$ is the number of eigenfunctions selected for the coarse 
neighborhood $\omega_{i}$. Notice that $\widehat{\boldsymbol{v}}_{\text {off }} \in V_{\text {off }}^{0}$. We can further write (2.28) as

$$
\begin{aligned}
\int_{D} \kappa^{-1}\left(v_{h}-v_{H}\right) \cdot w_{H}-\int_{D} \operatorname{div}\left(w_{H}\right)\left(\widehat{p}-p_{H}\right) & =0, \quad \forall w_{H} \in V_{\mathrm{off}}^{0}, \\
\int_{D} \operatorname{div}\left(\widehat{\boldsymbol{v}}_{\mathrm{off}}-v_{H}\right) q_{H} & =\int_{D} \operatorname{div}\left(\widehat{\boldsymbol{v}}_{\mathrm{off}}-\widehat{\boldsymbol{v}}\right) q_{H}, \quad \forall q_{H} \in Q_{H} .
\end{aligned}
$$

Taking $\boldsymbol{w}_{H}=\widehat{\boldsymbol{v}}_{\mathrm{off}}-\boldsymbol{v}_{H}$ and $q_{H}=\widehat{p}-p_{H}$ in (2.31), and adding the resulting equations, we obtain

$$
\int_{\Omega} \kappa^{-1}\left(\boldsymbol{v}_{h}-\boldsymbol{v}_{H}\right) \cdot\left(\widehat{\boldsymbol{v}}_{\mathrm{off}}-\boldsymbol{v}_{H}\right)=\int_{\Omega} \operatorname{div}\left(\widehat{\boldsymbol{v}}_{\mathrm{off}}-\widehat{\boldsymbol{v}}\right)\left(\widehat{p}-p_{H}\right)
$$

By the inf-sup condition (2.23) and the error equation (2.31), we have

$$
\left\|\widehat{p}-p_{H}\right\|_{L^{2}(\Omega)} \preceq C_{\text {infsup }}\left\|v_{h}-v_{H}\right\|_{\kappa^{-1}, \Omega} .
$$

Moreover, by the definition of the spectral problem (2.9), we have

$$
\int_{\Omega}\left(\operatorname{div}\left(\widehat{\boldsymbol{v}}_{\text {off }}-\widehat{\boldsymbol{v}}\right)\right)^{2} \preceq \sum_{i=1}^{N_{0}} \int_{\omega_{i}}\left(\operatorname{div}\left(\widehat{\boldsymbol{v}}_{\text {off }}-\widehat{\boldsymbol{v}}\right)\right)^{2} \preceq \sum_{i=1}^{N_{0}} s_{i}\left(\widehat{\boldsymbol{v}}_{\text {off }}-\widehat{\boldsymbol{v}}, \widehat{\boldsymbol{v}}_{\text {off }}-\widehat{\boldsymbol{v}}\right) .
$$

We can then derive from (2.32) the following

$$
\left\|\boldsymbol{v}_{h}-\boldsymbol{v}_{H}\right\|_{\kappa^{-1}, \Omega}^{2} \preceq\left\|\widehat{\boldsymbol{v}}_{\text {off }}-\boldsymbol{v}_{h}\right\|_{\kappa^{-1}, \Omega}^{2}+C_{\text {infsup }}^{2} \sum_{i=1}^{N_{0}} s_{i}\left(\widehat{\boldsymbol{v}}_{\mathrm{Off}}-\widehat{\boldsymbol{v}}, \widehat{\boldsymbol{v}}_{\mathrm{Off}}-\widehat{\boldsymbol{v}}\right) .
$$

Using the triangle inequality $\left\|\widehat{\boldsymbol{v}}_{\text {off }}-\boldsymbol{v}_{h}\right\|_{\kappa^{-1}, \Omega} \leq\left\|\widehat{\boldsymbol{v}}_{\text {off }}-\widehat{\boldsymbol{v}}\right\|_{\kappa^{-1}, \Omega}+\left\|\widehat{\boldsymbol{v}}-\boldsymbol{v}_{h}\right\|_{\kappa^{-1}, \Omega}$ and

$$
\left\|\widehat{\boldsymbol{v}}_{\mathrm{off}}-\widehat{\boldsymbol{v}}\right\|_{\kappa^{-1}, \Omega}^{2} \preceq \sum_{i=1}^{N_{0}}\left\|\widehat{\boldsymbol{v}}_{\mathrm{off}}-\widehat{\boldsymbol{v}}\right\|_{\kappa^{-1}, \omega_{i}}^{2} \preceq \sum_{i=1}^{N_{0}} s_{i}\left(\widehat{\boldsymbol{v}}_{\mathrm{off}}-\widehat{\boldsymbol{v}}, \widehat{\boldsymbol{v}}_{\mathrm{off}}-\widehat{\boldsymbol{v}}\right),
$$

we obtain

$$
\left\|\boldsymbol{v}_{h}-\boldsymbol{v}_{H}\right\|_{\mathcal{K}^{-1}, \Omega}^{2} \preceq\left\|\widehat{\boldsymbol{v}}-\boldsymbol{v}_{h}\right\|_{\mathcal{K}^{-1}, \Omega}^{2}+C_{\text {infsup }}^{2} \sum_{i=1}^{N_{0}} s_{i}\left(\widehat{\boldsymbol{v}}_{\text {off }}-\widehat{\boldsymbol{v}}, \widehat{\boldsymbol{v}}_{\text {off }}-\widehat{\boldsymbol{v}}\right) .
$$

The first term on the right hand side of (2.33) can be estimated by Lemma 2.5.1. For the second term on the right hand side of (2.33), by (2.29)-(2.30) and the fact 
that $\boldsymbol{\psi}_{k}^{i}$ 's are eigenfunctions of (2.8), we have

$$
s_{i}\left(\widehat{\boldsymbol{v}}_{\mathrm{off}}-\widehat{\boldsymbol{v}}, \widehat{\boldsymbol{v}}_{\mathrm{off}}-\widehat{\boldsymbol{v}}\right)=\sum_{k=l_{i}+1}^{J_{i}}\left(\lambda_{k}^{(i)}\right)^{-1}\left(\widehat{\boldsymbol{v}}_{i k}\right)^{2} a_{i}\left(\boldsymbol{\psi}_{k}^{i}, \boldsymbol{\psi}_{k}^{i}\right) .
$$

By the ordering of the eigenvalues (2.11) and orthogonality of eigenfunctions, we obtain

$$
s_{i}\left(\widehat{\boldsymbol{v}}_{\mathrm{off}}-\widehat{\boldsymbol{v}}, \widehat{\boldsymbol{v}}_{\mathrm{off}}-\widehat{\boldsymbol{v}}\right) \leq\left(\lambda_{l_{i}+1}^{(i)}\right)^{-1} a_{i}\left(\widehat{\boldsymbol{v}}_{\mathrm{off}}-\widehat{\boldsymbol{v}}, \widehat{\boldsymbol{v}}_{\mathrm{off}}-\widehat{\boldsymbol{v}}\right) \leq\left(\lambda_{l_{i}+1}^{(i)}\right)^{-1} a_{i}(\widehat{\boldsymbol{v}}, \widehat{\boldsymbol{v}})
$$

Combining the above results, we have

$$
\sum_{i=1}^{N_{0}} s_{i}\left(\widehat{\boldsymbol{v}}_{\mathrm{Off}}-\widehat{\boldsymbol{v}}, \widehat{\boldsymbol{v}}_{\mathrm{off}}-\widehat{\boldsymbol{v}}\right) \leq \sum_{i=1}^{N_{0}}\left(\lambda_{l_{i}+1}^{(i)}\right)^{-1} a_{i}(\widehat{\boldsymbol{v}}, \widehat{\boldsymbol{v}}) .
$$

This completes the proof.

We remark that in the error estimate (2.26), the first and second terms on the right-hand-side represent the errors due to the spectral basis functions and the coarse grid discretization, respectively.

\subsection{Oversampling approach}

One can use an oversampling approach to improve the accuracy of the method. The main idea of the oversampling method is to use larger domains to compute snapshots. Furthermore, performing POD in the snapshot space, we can achieve a lower dimensional approximation space. Oversampling technique can be particularly helpful for problems with scale separation. This is because by taking the restriction of the local solutions in larger domains in the interior, we avoid the pollution effects near the boundaries.

Let $D$ be a conforming subset of $\Omega$. By conforming subset, we mean that $D$ is 
formed by the union of connected fine grid elements. For a given function $\psi$ defined on $\partial D$, let $\left(\mathscr{H}_{D}(\psi), \xi_{D}\right) \in V_{h}(D) \times Q_{h}(D)$ be the solution of the weak form of the following problem

$$
\begin{aligned}
\kappa^{-1} \mathscr{H}_{D}(\psi)+\nabla \xi_{D} & =0 \quad \text { in } \quad D, \\
\operatorname{div}\left(\mathscr{H}_{D}(\psi)\right)=c_{D} & \text { in } \quad D, \\
\mathscr{H}_{D}(\psi) \cdot \boldsymbol{n}=\psi & \text { on } \quad \partial D,
\end{aligned}
$$

where $c_{D}=|D|^{-1} \int_{\partial D} \psi, \boldsymbol{V}_{h}(D)$ and $Q_{h}(D)$ are the restrictions of $\boldsymbol{V}_{h}$ and $Q_{h}$ to $D$ respectively. We call $\mathscr{H}_{D}(\psi)$ the $\kappa$-harmonic extension of $\psi$ in $D$.

Let $E_{i} \in \mathscr{E}_{H}$ be an interior coarse edge, and let $\omega_{i}^{+}$be a conforming subset of $D$ with $E_{i}$ lying in the interior of $\omega_{i}^{+}$, see Figure 1.1 for an example of $\omega_{i}^{+}$. Let $W_{i}\left(\partial \omega_{i}^{+}\right)$be the set of all piecewise constant functions defined on $\partial \omega_{i}^{+}$with respect to the fine grid partition. Consider the following set of functions defined on $E_{i}$

$$
\left\{\left.\mathscr{H}_{\omega_{i}^{+}}\left(\psi_{j}\right) \cdot \boldsymbol{m}_{i}\right|_{E_{i}} \quad, \quad \psi_{j} \in W_{i}\left(\partial \omega_{i}^{+}\right)\right\}
$$

By performing a standard POD on the above space, and selecting the first $l_{i}^{+}$dominant modes $\psi_{j}^{i, \text { ovs }}$, we obtain the following space

$$
\boldsymbol{V}_{\mathrm{ovs}}\left(E_{i}\right)=\operatorname{span}\left\{\boldsymbol{\psi}_{j}^{i, \text { ovs }}, 1 \leq j \leq l_{i}^{+}\right\}
$$

where the basis functions $\boldsymbol{\psi}_{j}^{i, \text { ovs }}$ are obtained by solving (2.5) with the boundary condition (2.6) replaced by $\boldsymbol{\psi}_{j}^{i, \text { ovs }} \cdot \boldsymbol{m}_{i}=\boldsymbol{\psi}_{j}^{i, \text { ovs }}$ on $E_{i}$. We call this local oversampling space. The oversampling space $V_{\text {ovs }}$ is obtained by the linear span of all local oversampling spaces. To obtain a numerical solution, we solve (2.2) with $\boldsymbol{V}_{H}=\boldsymbol{V}_{\text {ovs }}$.

Next, we discuss the outline of the convergence analysis for the oversampling 
approach. For any $\boldsymbol{v}_{h} \in V_{h}$ and for every $E_{i} \in \mathscr{E}_{H}$, we define $\mu_{E_{i}}$ as

$$
\mu_{E_{i}}=\left.\mathscr{H}_{\omega_{i}^{+}}\left(\left.\boldsymbol{v}_{h} \cdot \boldsymbol{n}\right|_{\partial \omega_{i}^{+}}\right) \cdot \boldsymbol{m}_{i}\right|_{E_{i}}
$$

which is the normal component on $E_{i}$ of the $\boldsymbol{\kappa}$-harmonic extension of $\boldsymbol{v}_{h} \cdot \boldsymbol{n}$ in the oversampled region $\omega_{i}^{+}$. Using $\mu_{E_{i}}$, we can then define $\widetilde{\boldsymbol{v}} \in \boldsymbol{V}_{\text {snap }}$ by

$$
\widetilde{\boldsymbol{v}}=\sum_{i=1}^{N_{e}} \mu_{E_{i}} \boldsymbol{\phi}_{0}^{i}
$$

where $\boldsymbol{\phi}_{0}^{i} \in \boldsymbol{V}_{\text {snap }}^{i}$ satisfies $\boldsymbol{\phi}_{0}^{i} \cdot \boldsymbol{m}_{i}=1$ on $E_{i}$. Next, we have

$$
\begin{aligned}
\|\widehat{\boldsymbol{v}}-\widetilde{\boldsymbol{v}}\|_{\mathcal{K}^{-1}, \Omega}^{2} & =\sum_{i=1}^{N_{e}}\left\|\left(\widehat{\boldsymbol{v}} \cdot \boldsymbol{m}_{i}\right) \boldsymbol{\phi}_{0}^{i}-\mu_{E_{i}} \boldsymbol{\phi}_{0}^{i}\right\|_{\mathcal{K}^{-1}, \omega_{i}}^{2} \\
& \preceq \sum_{i=1}^{N_{e}} H\left\|\left(\widehat{\boldsymbol{v}} \cdot \boldsymbol{m}_{i}\right)-\mu_{E_{i}}\right\|_{L^{2}\left(E_{i}\right)}^{2} \preceq H \delta,
\end{aligned}
$$

where we assumed that $H\left\|\left(\widehat{\boldsymbol{v}} \cdot \boldsymbol{m}_{i}\right)-\mu_{E_{i}}\right\|_{L^{2}\left(E_{i}\right)}^{2} \preceq H \boldsymbol{\delta}$ and $\widehat{\boldsymbol{v}} \cdot \boldsymbol{m}_{i}$ is the normal trace of $\widehat{\boldsymbol{v}}$ on $E_{i}$. If the forcing is constant within the union of $\omega_{i}^{+}$and $\omega_{i}$, then $\delta=0$. Otherwise, this value depends on the smoothness of $\kappa$ and $f$. For homogenization problems, one can show that $\delta$ is small.

Next, we choose an appropriate interpolant $\widetilde{\boldsymbol{v}}_{\text {ovs }}$ and compare it with $\widetilde{\boldsymbol{v}}$. Note that, we can write

$$
\widetilde{\boldsymbol{v}}_{\mathrm{ovs}}=\sum_{i=1}^{N_{e}} \sum_{j=1}^{l_{i}^{+}} c_{j}^{i} \boldsymbol{\psi}_{j}^{i, \mathrm{ovs}}
$$

for some constants $c_{j}^{i}$. Therefore,

$$
\begin{aligned}
\left\|\widetilde{\boldsymbol{v}}-\widetilde{\boldsymbol{v}}_{\mathrm{ovs}}\right\|_{\mathcal{K}^{-1}, \Omega}^{2} & =\left\|\sum_{i=1}^{N_{e}}\left(\mu_{E_{i}} \boldsymbol{\phi}_{0}^{i}-\sum_{j=1}^{l_{i}^{+}} c_{j}^{i} \boldsymbol{\psi}_{j}^{i, \mathrm{ovs}}\right)\right\|_{\mathcal{K}^{-1}, \Omega}^{2} \\
& \preceq \sum_{i=1}^{N_{e}} H\left\|\mu_{E_{i}}-\sum_{j=1}^{l_{i}^{+}} c_{j}^{i} \boldsymbol{\psi}_{j}^{i, \mathrm{ovs}} \cdot \boldsymbol{m}_{i}\right\|_{L^{2}\left(E_{i}\right)}^{2} .
\end{aligned}
$$

Denote by $\Phi^{E}$, the restriction of the snapshots on the edge $E$. We would like to find a 
reduced dimensional representation of $\Phi^{E}$ such that $\left\|\Phi^{E}-\Psi_{r}^{E} C_{r}\right\|$ is small, where $\Psi_{r}^{E}$ is the reduced-dimensional representation (the matrix of the size $N^{e} \times N^{r}$ ), where $N^{r}$ is the reduced dimension and $C^{r}$ is the matrix of the size $N^{r} \times N^{\partial \omega_{i}^{+}}$, where $N^{\partial \omega_{i}^{+}}$is the number of fine-grid edges on $\partial \omega_{i}^{+}$. This is achieved by POD as described above and we have $\left\|\Phi^{E}-\Psi_{r}^{E} C_{r}\right\|_{E} \leq 1 / \lambda_{l_{i}^{+}+1}^{+}$. From here, one can show that given values of the velocity $z$ on the boundary of $\partial \omega_{i}^{+}$, we have $\left\|\Phi^{E} z-\Psi_{r}^{E} C_{r} z\right\|_{2} \leq\left(1 / \lambda_{l_{i}^{+}+1}^{+}\right)\|z\|_{2}$. Combining these estimates, we have

$$
\left\|\widetilde{\boldsymbol{v}}-\widetilde{\boldsymbol{v}}_{\mathrm{OVS}}\right\|_{\kappa^{-1}, \Omega}^{2} \preceq \sum_{i=1}^{N_{e}} \frac{1}{\lambda_{l_{i}^{+}+1}^{+}} H\left\|\boldsymbol{v}_{h}\right\|_{L^{2}\left(\partial \omega_{i}^{+}\right)}^{2} \preceq \frac{1}{\Lambda^{+}} \sum_{i=1}^{N_{e}} H\left\|\boldsymbol{v}_{h}\right\|_{L^{2}\left(\partial \omega_{i}^{+}\right)}^{2}
$$

where $\Lambda^{+}=\min \left\{\lambda_{l_{i}^{+}+1}^{+}\right\}$.

One can consider an alternative approach where the snapshot space is obtained by performing POD as described above. More precisely, we use $\boldsymbol{V}_{\text {ovs }}$ as the snapshot space that can have a lower dimension compared to the original snapshot space that corresponds to non-oversampling case. As a next step, we perform a spectral decomposition following the non-oversampling case by considering $\mathscr{H}_{K}\left(\boldsymbol{\psi}_{j}^{i, \text { ovs }}\right)$ as a snapshot space. We denote this snapshot space by $\boldsymbol{V}_{\mathrm{ovs}}^{R}$, where $R$ stands for reduced dimension. The main advantage of this approach is that a lower dimensional snapshot space is used in the spectral decomposition and this snapshot space allows achieving a low dimensional structure when the problem has a scale separation. The latter may not hold if we apply non-oversampling procedure. To obtain the convergence analysis, we show that for every $\boldsymbol{v}_{h} \in \boldsymbol{V}_{h}$, there exists $\mu_{E_{i}}^{R} \boldsymbol{\phi}_{0}^{i} \in \boldsymbol{V}_{\text {ovs }}^{R}$ in the snapshot 
space, such that

$$
\left\|\mu_{E_{i}}-\mu_{E_{i}}^{R}\right\|_{L^{2}\left(E_{i}\right)}^{2} \preceq 1 / \lambda_{l_{i}^{+}+1}^{+}
$$

where $1 / \lambda_{l_{i}^{+}+1}^{+}$is the lowest eigenvalue that the corresponding eigenvector is not included in the snapshot space. This follows from standard POD result which provides an estimate for $\left\|\Psi^{E}-\Phi_{r}^{E} C_{r}\right\|_{F} \leq 1 / \lambda_{l_{i}^{+}+1}^{+}$. Under this condition and using the fact that $\|A z\|_{2} \leq\|A\|_{F}\|z\|_{2}$, we obtain (2.38). Using this reduced snapshot space, we can repeat our previous argument in Section 2.5 and obtain the convergence rate. We remark here that the oversampling approach discussed in this section does not result in a non-conforming method and the velocity solution remain mass conservative on the fine grid. We note also that the decay of the inverse of the eigenvalues in the oversampling case is faster than the non-oversampling case. Hence, from our analysis, we expect the errors decay faster as well. To illustrate this, we compare the decay in two media: a channelized high contrast permeability and a periodic permeability (they are $\kappa_{1}$ and $\kappa_{p e r}$ in Section 2.7 , respectively). We plot $\log \left(1 / \lambda_{i}\right)$ in the case of oversampling and non-oversampling, see Figure 2.5. Note that the eigenvalue problems in the two cases are different, but we normalize them for the ease of comparison. We observe that decay in the oversampling case is faster than the non-oversampling case after the second eigenvalue. 


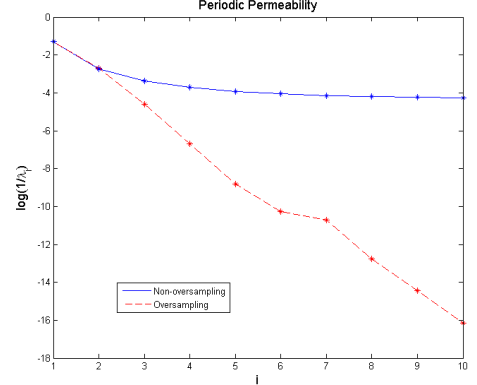

(a) $\kappa=\kappa_{p e r}$

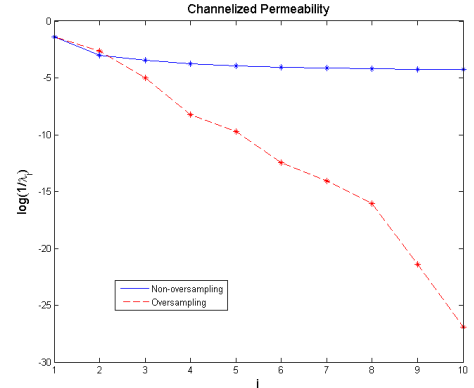

(b) $\kappa=\kappa_{1}$

Figure 2.5: Decay of $\log \left(1 / \lambda_{i}\right)$ for oversampling and non-oversampling.

\subsection{Numerical results}

In this section, we will present some numerical results to show the performance of the mixed GMsFEM (2.2) for approximating the flow problem (2.1). In all simulations reported below, the computational domain $\Omega=(0,1)^{2}$. The coarse grid $\mathscr{T}_{H}$ and the fine grid $\mathscr{T}_{h}$ are $N \times N$ and $n \times n$ uniform meshes, respectively. A fixed fine-grid size with $n=200$ is employed. Moreover, we will consider three different permeability fields $\kappa$, as depicted in Figure 2.6. These permeability fields have the same resolution

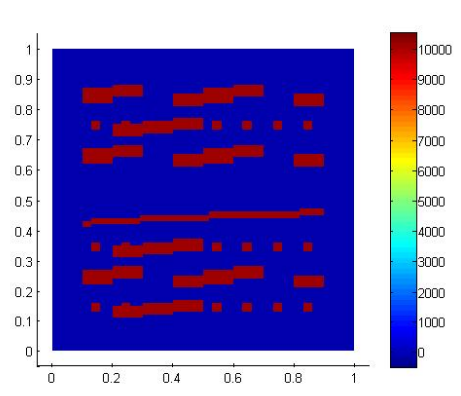

(a) $\kappa_{1}$

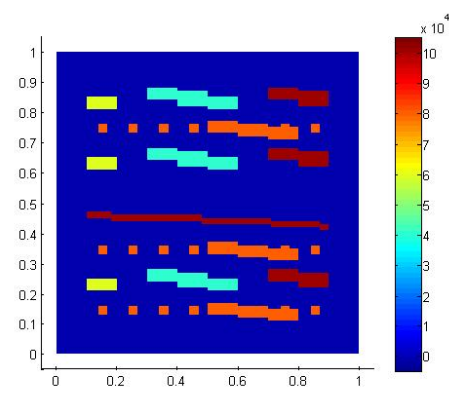

(b) $\kappa_{2}$

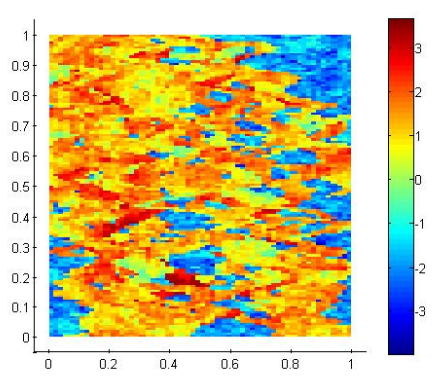

(c) $\kappa_{3}$ in $\log _{10}$ scale

Figure 2.6: Three permeability fields in the numerical experiments. 
as the fine-grid size. We will present the performance of the mixed GMsFEM for three types of applications; namely, we present single-phase flow problems, singlephase flow and transport problems, and two-phase flow and transport problems. To facilitate the presentation, we let $\left(\boldsymbol{v}_{f}, p_{f}\right),\left(\boldsymbol{v}_{s}, p_{s}\right)$ and $\left(\boldsymbol{v}_{o}, p_{o}\right)$ be the fine-grid solution, snapshot solution, and the GMsFEM solution respectively, where the snapshot solution is the solution of the discrete system (2.2) with all basis functions in the snapshot space are selected. Notice that the snapshot solution contains only the coarse-grid discretization error and the GMsFEM solution contains both coarse-grid and spectral errors, see Theorem 2.5.3. Furthermore, we define the following error quantities for the velocity field

$$
E_{o f}(v):=\left\|\boldsymbol{v}_{o}-\boldsymbol{v}_{f}\right\|_{\kappa^{-1}, \Omega} /\left\|\boldsymbol{v}_{f}\right\|_{\kappa^{-1}, \Omega}, E_{o s}(v):=\boldsymbol{v}_{o}-\boldsymbol{v}_{S}\left\|_{\kappa^{-1}, \Omega} /\right\| \boldsymbol{v}_{s} \|_{\kappa^{-1}, \Omega},
$$

which we term the total error and the spectral error, respectively. For pressure, we define the corresponding error quantities by

$$
E_{o f}(p):=\left\|p_{o}-p_{f}\right\|_{L^{2}(\Omega)} /\left\|p_{f}\right\|_{L^{2}(\Omega)}, E_{o s}(p):=\left\|p_{o}-p_{s}\right\|_{L^{2}(\Omega)} /\left\|p_{s}\right\|_{L^{2}(\Omega)}
$$

These error quantities are used to measure the performance of the mixed GMsFEM in the examples below.

\subsubsection{Single-phase flow}

We consider single-phase flow in this section. For the simulations, we will use two different coarse-mesh sizes with $N=10$ and $N=20$, called case 1 and case 2, respectively. The numerical results for the permeability fields $\kappa_{1}$ and $\kappa_{2}$, as well as 
the use of the above two spectral problems (2.9) and (2.10) are shown in Tables $2.1-$ 2.4. In these tables, the term "dof per $E$ " means the number of basis functions used for that coarse edge $E$. We remark that, for spectral problem 2, the first eigenfunction is always taken as the field with constant normal component on $E_{i}$. In Tables 2.1 - 2.2, the convergence behaviors of the method for the permeability field $\kappa_{1}$ are shown for cases 1 and 2, respectively. Notice that, cases 1 and 2 decompose each coarse-grid block as 20x20 and 10x10 grids, respectively. Therefore, for each coarse edge, there are 20 and 10 basis functions for cases 1 and 2 respectively. From these tables, we see clearly the convergence of the method when basis functions are added to the offline space. In addition, we see that the spectral errors $E_{o s}(v)$ and $E_{o s}(p)$ converge to machine precision. On the other hand, the total errors $E_{o f}(v)$ and $E_{o f}(p)$ converge to a fixed error when the number of basis functions are increased. This fixed error corresponds to the coarse grid discretization error and cannot be improved by introducing more spectral basis functions. Nevertheless, the coarse-grid error can be reduced by using a smaller coarse mesh size. This is confirmed numerically in Tables $2.1-2.2$. In particular, when $N=10$, the level of the coarse-grid error in velocity is about $2 \%$; and when $N=20$, the level of the coarse-grid error in velocity is reduced to about $0.5 \%$. We also observe a similar situation for pressure. Regarding the results for the permeability field $\kappa_{2}$, the results in Tables $2.3-2.4$ give a similar conclusion.

In Figure 2.7, we show the reciprocals of the eigenvalues for case 1 for the per- 
Table 2.1: Convergence of the offline solution, $\kappa=\kappa_{1}, n=200$ and $N=10$

\begin{tabular}{|c||c|c|c|c|c|c|c|c|}
\hline \multicolumn{1}{|c||}{} & \multicolumn{4}{c|}{ Spectral problem 1 } & \multicolumn{4}{c|}{ Spectral problem 2} \\
\hline dof per $E$ & $E_{\text {of }}(v)$ & $E_{o f}(p)$ & $E_{o s}(v)$ & $E_{o s}(p)$ & $E_{o f}(v)$ & $E_{o f}(p)$ & $E_{o s}(v)$ & $E_{o s}(p)$ \\
\hline 1 & 0.1331 & 0.0903 & 0.1329 & 0.0196 & 0.1523 & 0.1018 & 0.1525 & 0.0519 \\
3 & 0.0569 & 0.0896 & 0.0535 & 0.0031 & 0.0840 & 0.0902 & 0.0823 & 0.0133 \\
5 & 0.0308 & 0.0898 & 0.0229 & $5.78 \mathrm{e}-04$ & 0.0391 & 0.0898 & 0.0334 & 0.0031 \\
7 & 0.0236 & 0.0898 & 0.0112 & $1.39 \mathrm{e}-04$ & 0.0278 & 0.0898 & 0.0186 & 0.0010 \\
9 & 0.0210 & 0.0898 & 0.0026 & $7.18 \mathrm{e}-06$ & 0.0234 & 0.0898 & 0.0108 & $1.20 \mathrm{e}-04$ \\
11 & 0.0208 & 0.0898 & $9.53 \mathrm{e}-13$ & $4.87 \mathrm{e}-15$ & 0.0208 & 0.0898 & $3.92 \mathrm{e}-13$ & $4.94 \mathrm{e}-15$ \\
20 & 0.0208 & 0.0898 & $3.92 \mathrm{e}-13$ & $6.18 \mathrm{e}-15$ & 0.0208 & 0.0898 & $3.96 \mathrm{e}-13$ & $5.08 \mathrm{e}-15$ \\
\hline
\end{tabular}

Table 2.2: Convergence of the offline solution, $\kappa=\kappa_{1}, n=200$ and $N=20$

\begin{tabular}{|c||c|c|c|c|c|c|c|c|}
\hline \multicolumn{1}{|c||}{} & \multicolumn{4}{c|}{ Spectral problem 1} & \multicolumn{4}{c|}{ Spectral problem 2 } \\
\hline dof per $E$ & $E_{o f}(v)$ & $E_{o f}(p)$ & $E_{o s}(v)$ & $E_{o s}(p)$ & $E_{o f}(v)$ & $E_{o f}(p)$ & $E_{o s}(v)$ & $E_{o s}(p)$ \\
\hline 1 & 0.1788 & 0.0601 & 0.1792 & 0.0373 & 0.1551 & 0.0677 & 0.1554 & 0.0483 \\
2 & 0.0460 & 0.0486 & 0.0459 & 0.0023 & 0.0861 & 0.0507 & 0.0861 & 0.0155 \\
3 & 0.0251 & 0.0486 & 0.0246 & $6.68 \mathrm{e}-04$ & 0.0493 & 0.0488 & 0.0491 & 0.0055 \\
4 & 0.0115 & 0.0486 & 0.0102 & $1.15 \mathrm{e}-04$ & 0.0233 & 0.0486 & 0.0227 & 0.0016 \\
5 & 0.0054 & 0.0486 & $3.47 \mathrm{e}-12$ & $1.10 \mathrm{e}-14$ & 0.0054 & 0.0486 & $4.29 \mathrm{e}-12$ & $9.53 \mathrm{e}-15$ \\
10 & 0.0054 & 0.0486 & $1.56 \mathrm{e}-12$ & $1.29 \mathrm{e}-14$ & 0.0054 & 0.0486 & $4.82 \mathrm{e}-13$ & $9.61 \mathrm{e}-15$ \\
\hline
\end{tabular}

Table 2.3: Convergence of the offline solution, $\kappa=\kappa_{2}, n=200$ and $N=10$

\begin{tabular}{|c||c|c|c|c|c|c|c|c|}
\hline \multicolumn{1}{|c||}{} & \multicolumn{4}{c|}{ Spectral problem 1 } & \multicolumn{4}{c|}{ Spectral problem 2} \\
\hline dof per $E$ & $E_{\text {of }}(v)$ & $E_{\text {of }}(p)$ & $E_{o s}(v)$ & $E_{\text {os }}(p)$ & $E_{\text {of }}(v)$ & $E_{o f}(p)$ & $E_{o s}(v)$ & $E_{\text {os }}(p)$ \\
\hline 1 & 0.1404 & 0.0905 & 0.1403 & 0.0219 & 0.1482 & 0.0966 & 0.1482 & 0.0404 \\
3 & 0.0561 & 0.0894 & 0.0526 & 0.0030 & 0.0778 & 0.0900 & 0.0757 & 0.0121 \\
5 & 0.0266 & 0.0896 & 0.0168 & $3.04 \mathrm{e}-04$ & 0.0393 & 0.0897 & 0.0337 & 0.0047 \\
7 & 0.0232 & 0.0896 & 0.0105 & $1.20 \mathrm{e}-04$ & 0.0277 & 0.0896 & 0.0185 & 0.0017 \\
9 & 0.0209 & 0.0896 & 0.0022 & $5.35 \mathrm{e}-06$ & 0.0239 & 0.0896 & 0.0119 & $1.50 \mathrm{e}-04$ \\
11 & 0.0208 & 0.0896 & $8.35 \mathrm{e}-13$ & $8.19 \mathrm{e}-15$ & 0.0208 & 0.0896 & $2.46 \mathrm{e}-11$ & $7.48 \mathrm{e}-15$ \\
20 & 0.0208 & 0.0896 & $4.98 \mathrm{e}-13$ & $9.31 \mathrm{e}-15$ & 0.0208 & 0.0896 & $5.00 \mathrm{e}-13$ & $8.29 \mathrm{e}-15$ \\
\hline
\end{tabular}

meability field $\kappa_{1}$ and for a particular coarse-grid block. We also show the eigenvalue behavior for both spectral problems. From these figures, we see that the eigenvalues have a very sharp decay for the first 10 eigenvalues; and this behavior corresponds to the rapid decay in the solution errors shown in Table 2.1 and Table 2.3. Starting at 
Table 2.4: Convergence of the offline solution, $\kappa=\kappa_{2}, n=200$ and $N=20$

\begin{tabular}{|c||c|c|c|c|c|c|c|c|}
\hline \multicolumn{1}{|c||}{} & \multicolumn{4}{c|}{ Spectral problem 1 } & \multicolumn{4}{c|}{ Spectral problem 2 } \\
\hline dof per $E$ & $E_{o f}(v)$ & $E_{o f}(p)$ & $E_{o s}(v)$ & $E_{o s}(p)$ & $E_{o f}(v)$ & $E_{o f}(p)$ & $E_{o s}(v)$ & $E_{o s}(p)$ \\
\hline 1 & 0.1880 & 0.0616 & 0.1884 & 0.0405 & 0.1487 & 0.0636 & 0.1490 & 0.0428 \\
2 & 0.0427 & 0.0481 & 0.0425 & 0.0020 & 0.0833 & 0.0522 & 0.0833 & 0.0211 \\
3 & 0.0210 & 0.0481 & 0.0203 & $4.48 \mathrm{e}-04$ & 0.0528 & 0.0490 & 0.0527 & 0.0099 \\
4 & 0.0107 & 0.0481 & 0.0092 & $9.35 \mathrm{e}-05$ & 0.0272 & 0.0482 & 0.0267 & 0.0027 \\
5 & 0.0054 & 0.0481 & $6.57 \mathrm{e}-11$ & $1.12 \mathrm{e}-14$ & 0.0054 & 0.0481 & $1.45 \mathrm{e}-11$ & $5.72 \mathrm{e}-15$ \\
10 & 0.0054 & 0.0481 & $4.21 \mathrm{e}-12$ & $7.39 \mathrm{e}-15$ & 0.0054 & 0.0481 & $8.20 \mathrm{e}-12$ & $7.56 \mathrm{e}-14$ \\
\hline
\end{tabular}

the 11th eigenvalue, there is no decay any more. This situation signifies that we do not need any additional basis function. In particular, the first 11 eigenfunctions are enough to achieve a machine precision spectral error, as confirmed in Tables 2.1 and 2.3. We observe a very good correlation (0.99) between the error and the eigenvalue behavior.

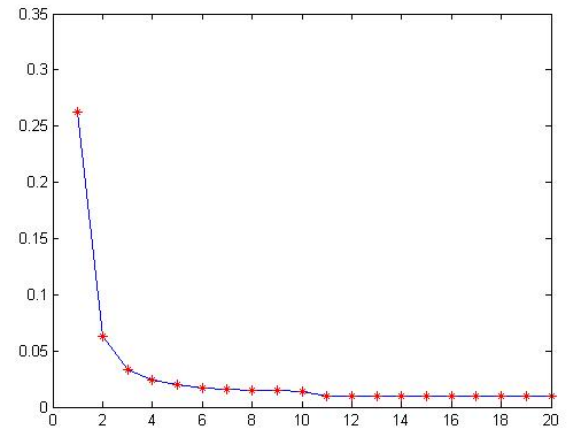

(a) Spectral problem 1

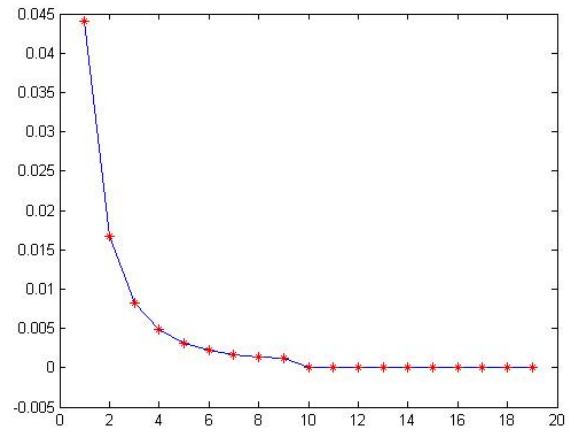

(b) Spectral problem 2

Figure 2.7: Inverse of eigenvalue $(1 / \lambda)$ behavior for the two spectral problems.

In order to see the performance of the postprocessing technique discussed in Section 2.4, we repeat the experiments corresponding to Table 2.1 and compute the 
postprocessed velocity, denoted as $\boldsymbol{v}_{p}$. We define $E_{p f}(v)=\left\|\boldsymbol{v}_{p}-\boldsymbol{v}_{f}\right\|_{\mathcal{K}^{-1}, \Omega} /\left\|\boldsymbol{v}_{f}\right\|_{\mathcal{K}^{-1}, \Omega}$. The numerical results are shown in Table 2.5. From these results, we clearly see that the postprocessed velocity is much more accurate than the velocity without postprocessing.

Table 2.5: Comparison of velocity and postprocessed velocity, $\kappa=\kappa_{1}, n=200$ and $N=10$

\begin{tabular}{|c||c|c|c|c|}
\hline \multicolumn{1}{|c||}{} & \multicolumn{2}{c|}{ Spectral problem 1 } & \multicolumn{2}{c|}{ Spectral problem 2 } \\
\hline dof per $E$ & $E_{o f}(v)$ & $E_{p f}(v)$ & $E_{o f}(v)$ & $E_{p f}(v)$ \\
\hline 1 & 0.1331 & 0.1327 & 0.1523 & 0.1525 \\
3 & 0.0569 & 0.0536 & 0.0840 & 0.0823 \\
5 & 0.0308 & 0.0232 & 0.0391 & 0.0338 \\
7 & 0.0236 & 0.0118 & 0.0278 & 0.0190 \\
9 & 0.0210 & 0.0046 & 0.0234 & 0.0114 \\
11 & 0.0208 & 0.0037 & 0.0208 & 0.0037 \\
20 & 0.0208 & 0.0037 & 0.0208 & 0.0037 \\
\hline
\end{tabular}

We remark that one can also consider using the curl of the velocity in constructing the offline space. We have studied an offline space construction that uses

$$
a_{i}(\boldsymbol{\psi}, \boldsymbol{\phi})=\int_{\omega_{i}} \operatorname{curl}\left(\kappa^{-1} \boldsymbol{\psi}\right) \operatorname{curl}\left(\kappa^{-1} \boldsymbol{\phi}\right), \quad s_{i}(\boldsymbol{\psi}, \boldsymbol{\phi})=\int_{\omega_{i}} \kappa^{-1} \boldsymbol{\psi} \cdot \boldsymbol{\phi} .
$$

Table 2.6 shows the convergence of the numerical solution obtained by using this spectral problem. As observed, the numerical results are not as good as those shown earlier for velocity error and for small number of basis functions.

\subsubsection{Oversampling technique}

Our first numerical example uses periodic coefficients. Our main objective is to show that oversampling technique can identify the first-order corrector part of the 
Table 2.6: Convergence of the offline solution using the curl-based spectral problem, $\kappa=\kappa_{1}, n=200$ and $N=10$

\begin{tabular}{|c||c|c|c|c|}
\hline dof per $E$ & $E_{o f}(v)$ & $E_{o f}(p)$ & $E_{o s}(v)$ & $E_{o s}(p)$ \\
\hline 1 & 0.1523 & 0.1018 & 0.1525 & 0.0519 \\
3 & 0.1062 & 0.0994 & 0.1052 & 0.0447 \\
5 & 0.0996 & 0.0964 & 0.0984 & 0.0373 \\
7 & 0.0620 & 0.0902 & 0.0590 & 0.0108 \\
9 & 0.0367 & 0.0898 & 0.0305 & 0.0024 \\
11 & 0.0312 & 0.0898 & 0.0235 & 0.0013 \\
20 & 0.0208 & 0.0898 & $3.90 \mathrm{e}-13$ & $5.54 \mathrm{e}-15$ \\
\hline
\end{tabular}

solution and avoid boundary effects. We consider the coefficient

$$
\kappa_{\text {per }}\left(x_{1}, x_{2}\right)=\left\{\begin{array}{cl}
1+\Gamma\left(x_{1}, x_{2}\right) \Pi_{i=1}^{2}\left(0.4-\left|x_{i}-0.5\right|\right), & \text { if }\left(x_{1}, x_{2}\right) \in[0.1,0.9]^{2} \\
1, & \text { otherwise }
\end{array}\right.
$$

where

$$
\Gamma\left(x_{1}, x_{2}\right)=\frac{2+1.8 \sin \left(2 \pi x_{1} / \varepsilon\right)}{2+1.8 \sin \left(2 \pi x_{2} / \varepsilon\right)}+\frac{2+1.8 \sin \left(2 \pi x_{1} / \varepsilon\right)}{2+1.8 \cos \left(2 \pi x_{2} / \varepsilon\right)}
$$

We consider 4 cases. Case 1 . Use oversampling technique to construct the snapshot space. When constructing the snapshot space, we select the eigenvectors corresponding to the first $l_{i}^{+}$eigenvalues on each coarse edge and use these eigenvectors as our offline space. Case 2. Use oversampling technique to construct the snapshot space. When constructing the snapshot space, we select the eigenvectors corresponding to the first 3 eigenvalues on each coarse edge and perform spectral problem 1 (see Section 2.2.2) on this snapshot space and select the eigenvectors corresponding to first $l_{i}$ eigenvalues as our offline space. Case 3. Construct the snapshot space without oversampling technique. In this case, we perform spectral problem 1 and select the eigenvectors corresponding to first $l_{i}$ eigenvalues as our offline space. Case 4 . Con- 
struct the snapshot space without oversampling technique. In this case, we perform spectral problem 2 and select the eigenvectors corresponding to first $l_{i}$ eigenvalues as our offline space. Our numerical results presented in Table 2.7 show that oversampling technique does give a better performance compared without oversampling, in general. Besides, we can obtain a much smaller snapshot space using oversampling technique while the accuracy of the solution is similar (see cases 2 and 3).

Table 2.7: Comparison of the 4 cases (relative velocity error w.r.t. fine scale solution), $\kappa=\kappa_{\text {per }}, n=200, N=10$

\begin{tabular}{|c||c|c|c|c|}
\hline dof per $E$ & Case 1 & Case 2 & Case 3 & Case 4 \\
\hline 1 & 0.0882 & 0.0985 & 0.0987 & 0.0856 \\
2 & 0.0241 & 0.0192 & 0.0206 & 0.0305 \\
3 & 0.0189 & 0.0189 & 0.0204 & 0.0302 \\
\hline
\end{tabular}

Next, we consider the high contrast permeability field $\kappa_{1}$ and compare to the previous results, see Table 2.8. Again, we see that the error is reduced if we apply oversampling technique and the oversampling allows obtaining a small dimensional snapshot space.

Table 2.8: Comparison of the 4 cases (relative velocity error w.r.t. fine scale solution), $\kappa=\kappa_{1}, n=200, N=10$

\begin{tabular}{|c||c|c|c|c|}
\hline dof per $E$ & Case 1 & Case 2 & Case 3 & Case 4 \\
\hline 1 & 0.1336 & 0.1332 & 0.1331 & 0.1523 \\
2 & 0.0400 & 0.0920 & 0.0916 & 0.1201 \\
3 & 0.0234 & 0.0234 & 0.0569 & 0.0840 \\
\hline
\end{tabular}

The computational cost of the oversampling technique can be reduced signifi- 
cantly if one uses randomized boundary conditions to generate the snapshot space. The main idea of this approach is to use random boundary conditions and solve for local snapshot solutions. More precisely, if we want to construct $k$ multiscale basis for velocity on the coarse edge $E_{i}$, then we can impose $k+M$ random boundary conditions on $\partial \omega_{i}^{+}$, where $M$ is some fixed but small integer. Then we follow the same procedure as discussed in Section 2.6. The analysis of this approach can be found in [15]. For example, in the setting of case 1 in Tables 2.7 and 2.8, the dimension of boundary conditions on each $\partial \omega_{i}^{+}$is 120 . We conduct the same set of experiments (case 1) with some random boundary conditions and report the results in Tables 2.9 and 2.10. Note that in Tables 2.9 and $2.10, k$ is the number of dof per $E$.

Table 2.9: Use of randomized boundary conditions in oversampling technique (relative velocity error w.r.t. fine scale solution), $\kappa=\kappa_{p e r}, n=200, N=10$

\begin{tabular}{|c|c|c|c|c|}
\hline \# of random BC & $k+2$ & $k+4$ & $k+6$ & 120 (Case 1) \\
\hline 1 & 0.0895 & 0.0881 & 0.0875 & 0.0882 \\
\hline 2 & 0.0282 & 0.0250 & 0.0259 & 0.0241 \\
\hline 3 & 0.0206 & 0.0202 & 0.0200 & 0.0189 \\
\hline
\end{tabular}

Table 2.10: Use of randomized boundary conditions in oversampling technique (relative velocity error w.r.t. fine scale solution), $\kappa=\kappa_{1}, n=200, N=10$

\begin{tabular}{|c|c|c|c|c|}
\hline k $\quad$ \# of random $\mathrm{BC}$ & $k+2$ & $k+4$ & $k+6$ & 120 (Case 1) \\
\hline 1 & 0.1872 & 0.1893 & 0.1876 & 0.1331 \\
\hline 2 & 0.0761 & 0.0559 & 0.0500 & 0.0400 \\
\hline 3 & 0.0319 & 0.0283 & 0.0273 & 0.0234 \\
\hline
\end{tabular}


We can see that, in general, using a snapshot space from randomized boundary conditions leads to slightly larger errors. However, the computational cost is reduced substantially (in this example the cost is even less than the approach without oversampling). We remark here that we also tried to use randomized boundary conditions for the approach without oversampling, but we do not see good convergence.

\subsubsection{Single-phase flow and transport}

We will now consider simulating single-phase flow and transport problems by the mixed GMsFEM with spectral problem 1. Specifically, we consider flow with zero Neumann boundary condition

$$
\begin{aligned}
-\kappa \nabla p=\boldsymbol{v}, & \text { in } \Omega, \\
\operatorname{div} \boldsymbol{v}=f, & \text { in } \Omega, \\
\boldsymbol{v} \cdot \boldsymbol{n}=0, & \text { on } \partial \Omega .
\end{aligned}
$$

In addition, the saturation equation is given by

$$
S_{t}+\boldsymbol{v} \cdot \nabla S=r
$$

where $S$ is the saturation and $r$ is the source. The above flow equation is solved by the mixed GMsFEM, and the saturation equation is solved on the fine grid by the finite volume method. Let $S_{i}^{n}$ be the value of $S$ on the fine element $\tau_{i}$ at time $t_{n}$, where $t_{n}=t_{0}+n \Delta t, t_{0}$ is the initial time and $\Delta t$ is the time step size chosen according to CFL condition. Then, $S_{i}^{n}$ satisfies

$$
\left|\tau_{i}\right| \frac{S_{i}^{n+1}-S_{i}^{n}}{\Delta t}+\int_{\partial \tau_{i}} \hat{S}^{n}(\boldsymbol{v} \cdot \boldsymbol{n})=r_{i}\left|\tau_{i}\right|
$$

where $r_{i}$ is the average value of $r$ on $\tau_{i}$ and $\hat{S}^{n}$ is the upwind flux. 
In our simulations, we will take $f$ to be zero except for the top-left and bottomright fine-grid elements, where $f$ takes the values of 1 and -1 , respectively. Moreover, we set the initial value of $S$ to be zero. For the source $r$, we also take it as zero except for the top-left fine element where $r=1$.

In Figures $2.8-2.11$, the saturation plots, shown from left to right, refer to the simulations at different times, namely; $t=1000,3000$, and 5000. The saturation plots in Figure 2.8 are obtained by using the fine-scale velocity $\boldsymbol{v}_{f}$ in (2.40). We denote these saturations $S_{f}$. Similarly, the saturation plots in Figures $2.9-2.11$ are obtained by using the multiscale velocity $\boldsymbol{v}_{o}$ in (2.40). We denote these saturations $S_{o}$. When selecting the multiscale basis functions, we use the first spectral problem (2.9). In order to see the effect of using a different number of multiscale basis functions on each coarse edge, we repeat the simulation with different settings. In the figures, the relative $L^{2}$ error refers to the relative $L^{2}$ error of the saturation. We compute this as

$$
\text { Relative error }=\frac{\left\|S_{o}-S_{f}\right\|_{L^{2}(\Omega)}}{\left\|S_{f}\right\|_{L^{2}(\Omega)}} .
$$

In addition, we use a $10 \times 10$ coarse grid for all simulations.

From Figure 2.9, we see that if only one multiscale basis functions are used on each coarse edge, the relative $L^{2}$ error of the saturation is about $4 \%$ to $9 \%$. Note that, in this case, the dimension of the velocity space $\boldsymbol{V}_{\text {off }}^{0}$ is only about $0.5 \%$ of that of the fine scale velocity space $\boldsymbol{V}_{h}^{0}$. This shows that the mixed generalized multiscale finite element space has a very good approximation property. We can further reduce the relative error of saturation by using more basis functions per coarse edge. In 
Figs. 2.10 and 2.11, we present the relative errors for saturation when 3 and 5 basis functions are used per edge respectively. We see that the errors are reduced to approximately $2 \%$. In these cases, the dimensions of the velocity space $\boldsymbol{V}_{\text {off }}^{0}$ are increased slightly to $1 \%$ and $1.4 \%$ of the fine scale velocity space $\boldsymbol{V}_{h}^{0}$, respectively.

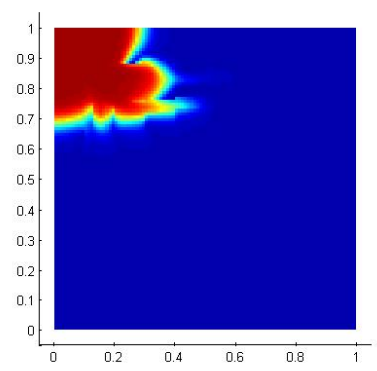

(a) $t=1000$

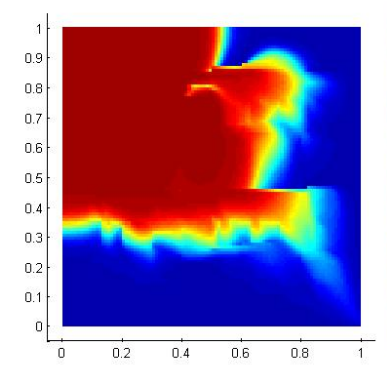

(b) $t=3000$

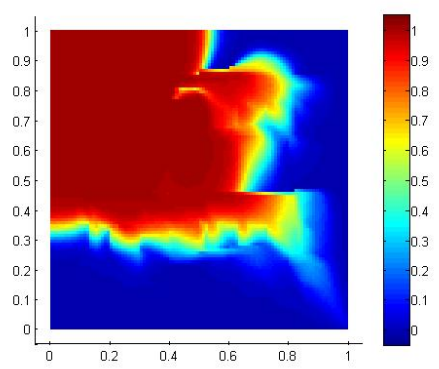

(c) $t=5000$

Figure 2.8: Saturation solution obtained by using $v_{f}$ in (2.40)

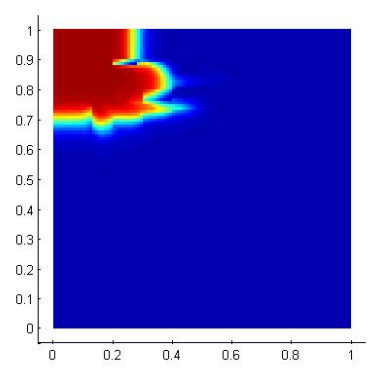

(a) Rel. $L^{2}$ err. $=9.0 \%$

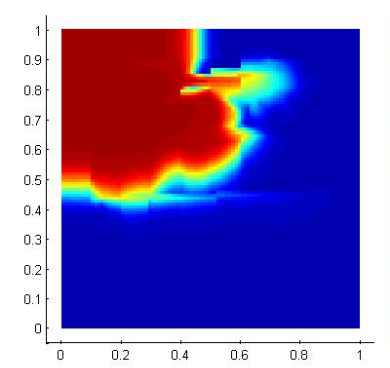

(b) Rel. $L^{2}$ err. $=6.4 \%$

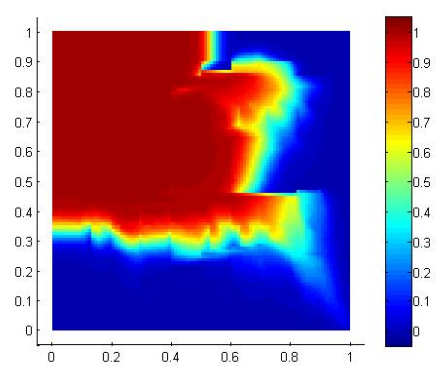

(c) Rel. $L^{2}$ err. $=4.4 \%$

Figure 2.9: Saturation solution obtained by using $v_{o}(10 \times 10$ coarse grid, 1 basis per coarse edge) in (2.40) 


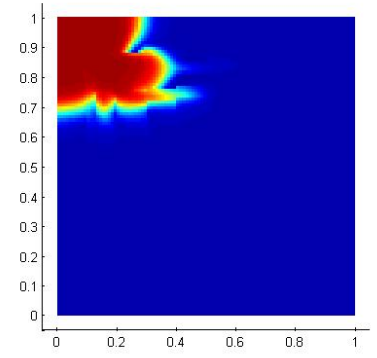

(a) Rel. $L^{2}$ err. $=2.0 \%$

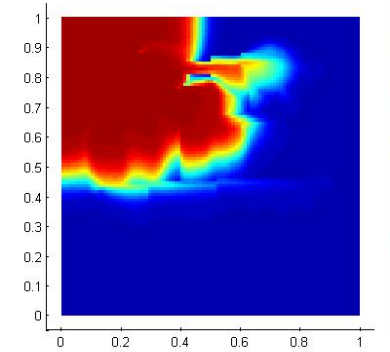

(b) Rel. $L^{2}$ err. $=1.3 \%$

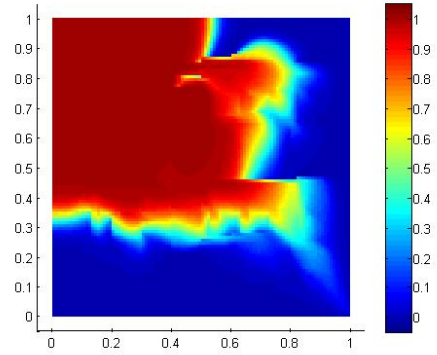

(c) Rel. $L^{2}$ err. $=0.8 \%$

Figure 2.10: Saturation solution obtained by using $v_{o}(10 \times 10$ coarse grid, 3 basis per coarse edge) in (2.40)

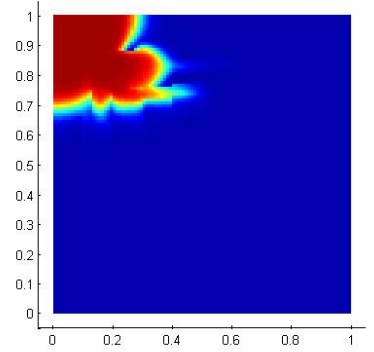

(a) Rel. $L^{2}$ err. $=2.0 \%$

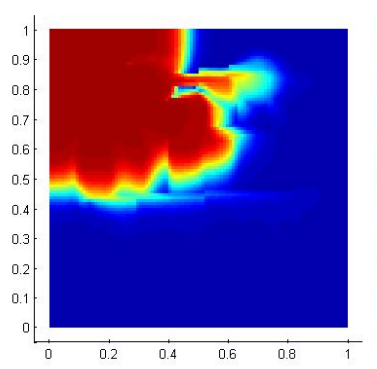

(b) Rel. $L^{2}$ err. $=0.8 \%$

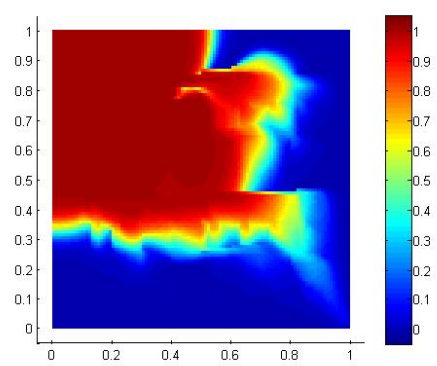

(c) Rel. $L^{2}$ err. $=0.5 \%$

Figure 2.11: Saturation solution obtained by using $v_{o}(10 \times 10$ coarse grid, 5 basis per coarse edge) in (2.40) 
2.7.4 Two-phase flow and transport

Finally, we present our simulation results for two-phase flow and transport problems. Consider the flow problem with zero Neumann boundary condition

$$
\begin{aligned}
& -\eta(S) \kappa \nabla p=\boldsymbol{v}, \quad \text { in } \Omega \\
& \operatorname{div} \boldsymbol{v}=f, \quad \text { in } \Omega \\
& \boldsymbol{v} \cdot \boldsymbol{n}=0, \quad \text { on } \partial \Omega,
\end{aligned}
$$

where

$$
\eta(S)=\frac{\kappa_{r w}(S)}{\mu_{w}}+\frac{\kappa_{r o}(S)}{\mu_{o}}
$$

and

$$
\kappa_{r w}(S)=S^{2}, \kappa_{r o}(S)=(1-S)^{2}, \mu_{w}=1, \mu_{o}=5 .
$$

The saturation equation is given by

$$
S_{t}+v \cdot \nabla F(S)=r
$$

where

$$
F(S)=\frac{\kappa_{r w}(S) / \mu_{w}}{\kappa_{r w}(S) / \mu_{w}+\kappa_{r o}(S) / \mu_{o}} .
$$

Adopting the same notations as in the single-phase flow case, we use the following discretization for saturation

$$
\left|\tau_{i}\right| \frac{S_{i}^{n+1}-S_{i}^{n}}{\Delta t}+\int_{\partial \tau_{i}} F\left(\hat{S}^{n}\right)(\boldsymbol{v} \cdot \boldsymbol{n})=g_{i}\left|\tau_{i}\right| .
$$

The source terms $f$ and $r$ are the same as in the single-phase case. For the construction of the offline space, we also use the spectral problem 1.

In Figures 2.12 - 2.15, the saturation plots, shown from left to right, refer to 


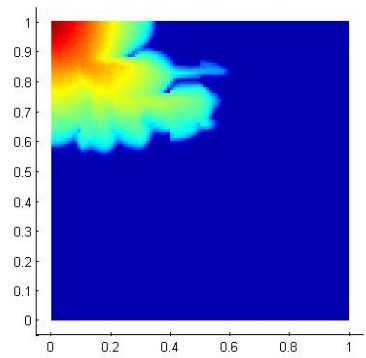

(a) $t=1000$

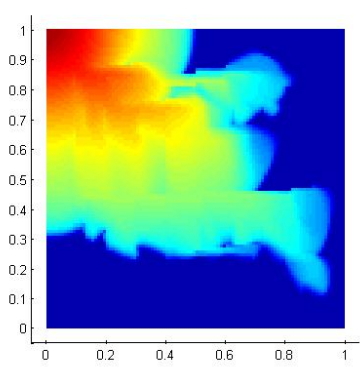

(b) $t=3000$

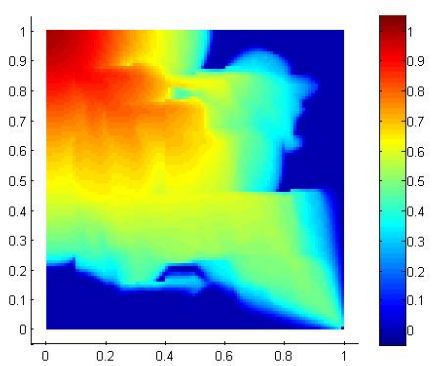

(c) $t=5000$

Figure 2.12: Saturation solution obtained by using $v_{f}$ in $(2.41)$

the simulations at different times; namely, $t=1000,3000$, and 5000. The saturation plots in Figure 2.12 are obtained by using the fine-scale velocity $v_{f}$ in $(2.41)$. We denote these saturations $S_{f}$. Similarly, the saturation plots in Figures $2.13-2.15$ are obtained by using the multiscale velocity $v_{o}$ in (2.41). Overall speaking, we observe error reductions from using 1 basis functions per edge to 5 basis functions per edge. In particular, for $t=1000$, the relative error reduces from $9.3 \%$ to $2.6 \%$ when using 5 basis functions per edge, and for $t=5000$, the relative error reduces from $5.5 \%$ to $1.3 \%$ when using 5 basis functions per edge.

In our last numerical example, we show the performance of our method when applying to a more realistic permeability field. We pick the top layer of the SPE10 permeability field (see Figure 2.6(c)) in the following set of experiments. The model is again the water and oil two-phase flow equations presented above. The permeability field is originally 220 by 60, and we project it into a fine grid of resolution 220 by 220 . Then, the coarse grid is set to be 11 by 11, which means the local grid is 10 by 10 in each coarse block. The saturation plots are depicted in Figures $2.16-2.19$. In this 


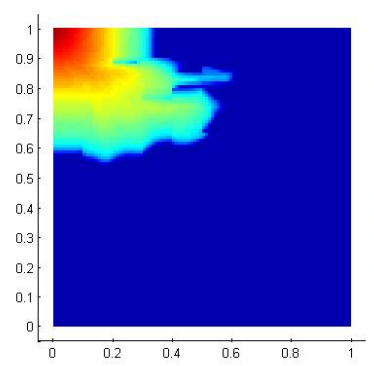

(a) Rel. $L^{2}$ err. $=9.3 \%$

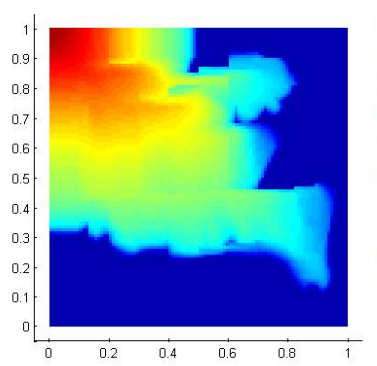

(b) Rel. $L^{2}$ err. $=5.9 \%$

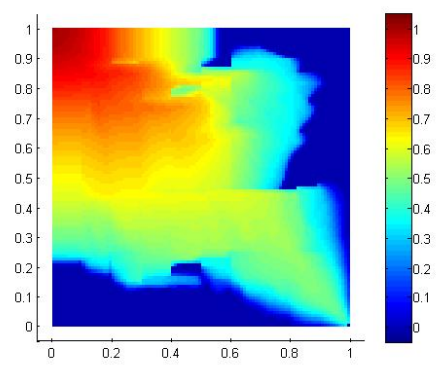

(c) Rel. $L^{2}$ err. $=5.5 \%$

Figure 2.13: Saturation solution obtained by using $v_{o}(10 \times 10$ coarse grid, 1 basis per coarse edge) in (2.41)

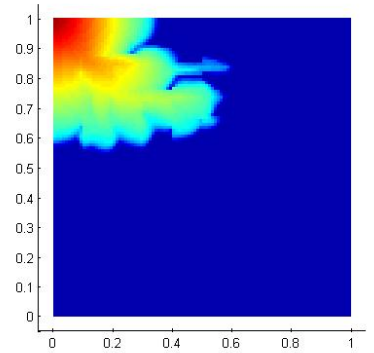

(a) Rel. $L^{2}$ err. $=2.8 \%$

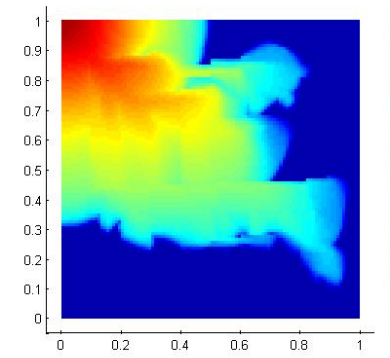

(b) Rel. $L^{2}$ err. $=1.6 \%$

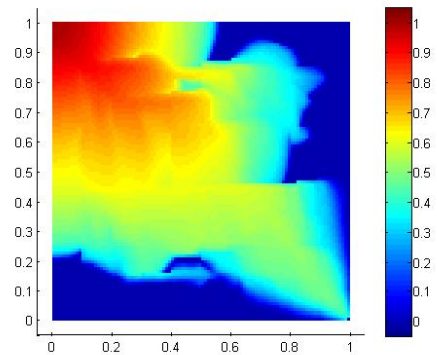

(c) Rel. $L^{2}$ err. $=1.6 \%$

Figure 2.14: Saturation solution obtained by using $v_{o}(10 \times 10$ coarse grid, 3 basis per coarse edge) in (2.41)

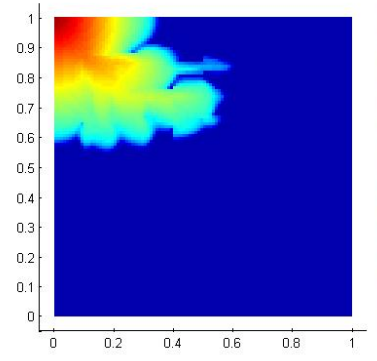

(a) Rel. $L^{2}$ err. $=2.6 \%$

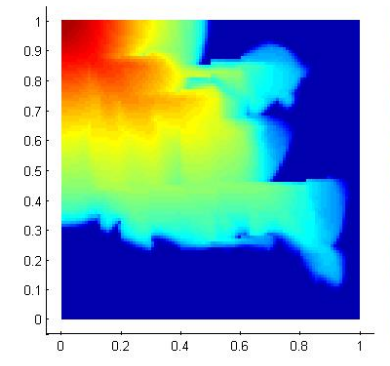

(b) Rel. $L^{2}$ err. $=1.4 \%$

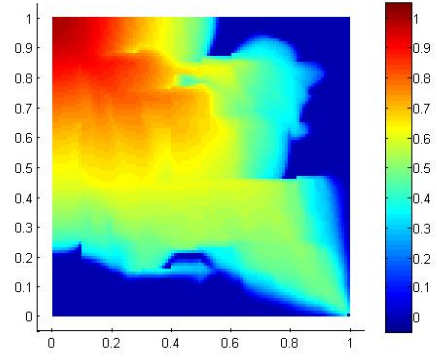

(c) Rel. $L^{2}$ err. $=1.3 \%$

Figure 2.15: Saturation solution obtained by using $v_{o}(10 \times 10$ coarse grid, 5 basis per coarse edge) in (2.41) 
example, we observe that, at first glance, the multiscale saturation solution looks similar to the fine solution if we use one multiscale basis function per edge. However, if we take a closer look, we notice some missing features in the water front. When we use four or six basis functions per coarse edge, these features can be recovered correctly. This shows the importance of these additional multiscale basis functions. More quantitatively, we observe more error reductions from using 1 basis functions per edge to 5 basis functions per edge compared with the previous examples. In particular, for $t=1000$, the relative error reduces from $18.8 \%$ to $3.6 \%$ when using 5 basis functions per edge. Likewise, for $t=5000$, the relative error reduces from $20.7 \%$ to $5.3 \%$ when using 5 basis functions per edge.

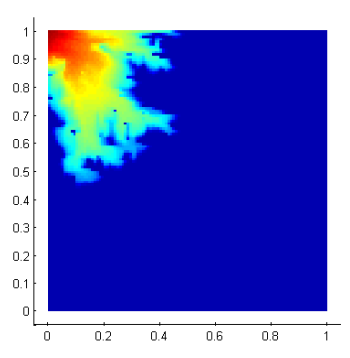

(a) $t=1000$

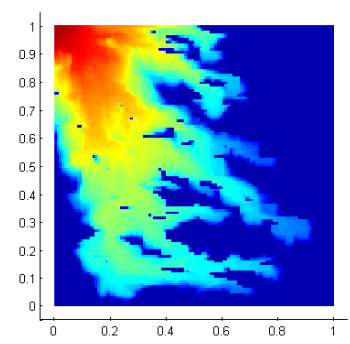

(b) $t=3000$

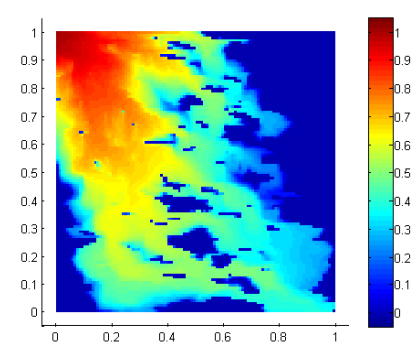

(c) $t=5000$

Figure 2.16: Saturation solution obtained by using $v_{f}$ in (2.41)

We have seen that mixed GMsFEM can provide accurate approximation for the saturation in the multiphase flow simulation. In fact, at the end of the day, what petroleum engineers care are some curves such as the water cut at producers. In our two-phase flow example, the water cut produced by different methods are depicted in 


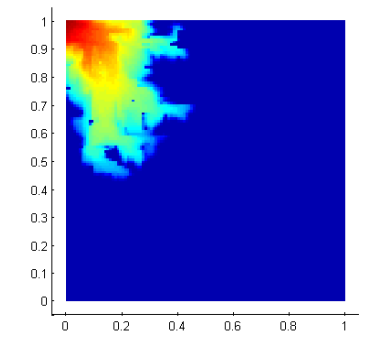

(a) Rel. $L^{2}$ err. $=18.8 \%$

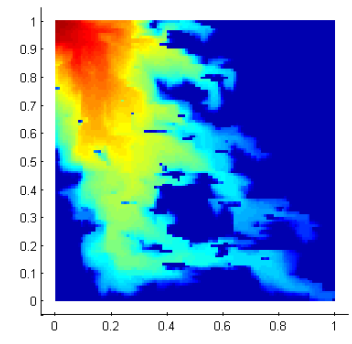

(b) Rel. $L^{2}$ err. $=25.4 \%$

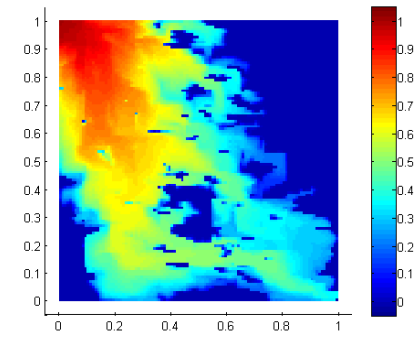

(c) Rel. $L^{2}$ err. $=20.7 \%$

Figure 2.17: Saturation solution obtained by using $v_{o}(11 \times 11$ coarse grid, 1 basis per coarse edge) in (2.41)

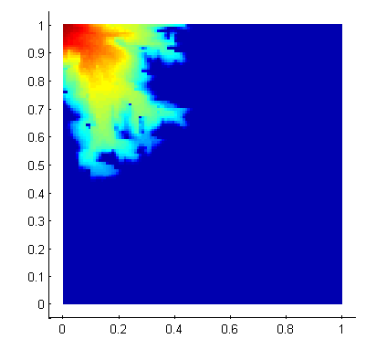

(a) Rel. $L^{2}$ err. $=5.2 \%$

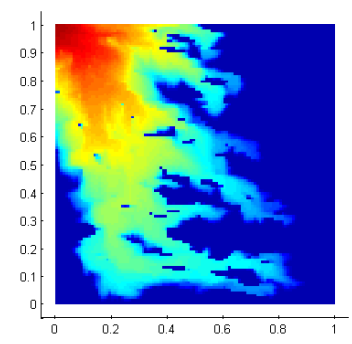

(b) Rel. $L^{2}$ err. $=10.2 \%$

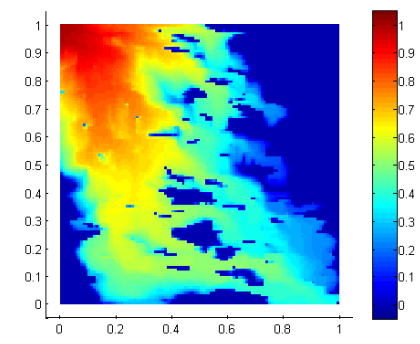

(c) Rel. $L^{2}$ err. $=7.6 \%$

Figure 2.18: Saturation solution obtained by using $v_{o}(11 \times 11$ coarse grid, 3 basis per coarse edge) in (2.41)

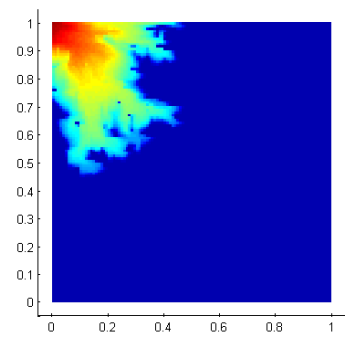

(a) Rel. $L^{2}$ err. $=3.6 \%$

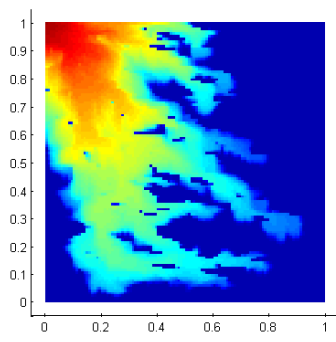

(b) Rel. $L^{2}$ err. $=4.5 \%$

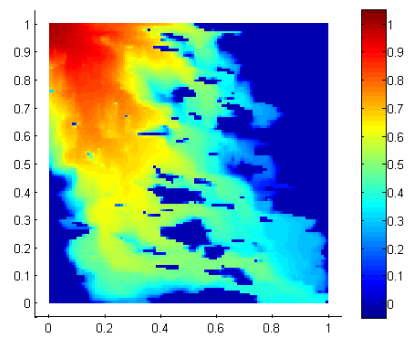

(c) Rel. $L^{2}$ err. $=5.3 \%$

Figure 2.19: Saturation solution obtained by using $v_{o}(11 \times 11$ coarse grid, 5 basis per coarse edge) in (2.41) 


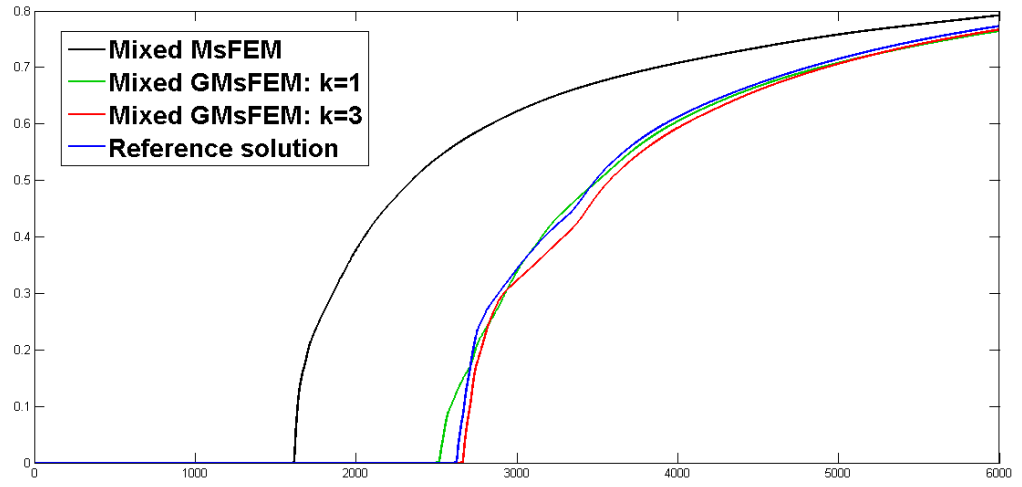

Figure 2.20: Water cut at producer for the two-phase flow example.

Figure 2.20. We observe that the water cut produced by mixed MsFEM is far from the reference solution. On the other hand, mixed GMsFEM produces very accurate approximation even with only 1 basis function per coarse edge, in which case the dimension of the coarse space is same as the mixed MsFEM.

Because the construction of the coarse space in mixed GMsFEM requires to solve many local boundary value problems and spectral problems, solving one pressure equation (2.1) using mixed GMsFEM on a coarse grid may be slower than simply solving the fine grid problem. However, in our targeting applications like reservoir simulations, the pressure equation needs to be solved many times. Since the coarse space constructed by mixed GMsFEM is very accurate, it can be reused throughout the simulation. Indeed, the saturation plots and the water cut in this section are generated without updating the coarse spaces. Consequently, the coarse multiscale space needs to be computed only once in the beginning of the simlution. Due to the cumulative saving in the solving time of the pressure equation, the total simulation 
Table 2.11: Two-phase flow example timing using different methods.

\begin{tabular}{|c|c|c|}
\hline & $\boldsymbol{V}_{H}$ construction time & Total simulation time \\
\hline Fine scale reference & - & $1123 \mathrm{~s}$ \\
Mixed MsFEM & $0.49 \mathrm{~s}$ & $510 \mathrm{~s}$ \\
Mixed GMsFEM $\left(l^{i}=1\right)$ & $1.05 \mathrm{~s}$ & $520 \mathrm{~s}$ \\
Mixed GMsFEM $\left(l^{i}=3\right)$ & $1.17 \mathrm{~s}$ & $667 \mathrm{~s}$ \\
\hline
\end{tabular}

time by applying mixed GMsFEM will be shorter than the full fine grid simulation. In Table 2.11, we list the actual simulation time for the two-phase flow example using different methods. We can see that the overhead due to the construction of the coarse multiscale function spaces is very tiny compared with the total simulation time. Because of the saving in solving the pressure equation, all the coarse scale simulations are about two time faster than the fine scale simulation (note that we only solve the pressure equation on a coarse grid, the transport equation is still solved on a fine grid). In summary, Figure 2.20 and Table 2.11 show that mixed GMsFEM produces more reliable solution than mixed MsFEM, while the simulation time of mixed GMsFEM is similar to mixed MsFEM (but both are much faster than fine grid simulation). Hence, mixed GMsFEM is a very attractive alternative for reservoir simulations. 


\section{MIXED GMSFEM FOR PLANAR LINEAR ELASTICITY}

The main objective of multiscale modeling for composite materials is to predict the macroscopic behavior of some composites composed of materials with different material properties. In particular, the stress distribution experienced by the material is oftentimes of interest. In this section, we will focus on the mixed formulation of the planar elasticity systems, where the stress tensor is one of the unknown. Mixed formulation are attractive because it is robust for nearly incompressible materials and the solution conserves energy when modeling elastic waves.

Previously, some multiscale methods for the linear elasticity equations were studied $[13,23]$. These methods were developed for the $\boldsymbol{H}^{1}$-elliptic displacement formulation of the elasticity equations. It is well-known that when modeling nearly incompressible material, the numerical error of the solution in the displacement formulation can be significantly large (the so-called locking effect) [8]. Mixed methods are good alternatives as they are robust against locking. Another advantage of mixed methods is the direct computation of the stress tensor, which is usually the physical quantity of interest in industrial applications. One common type of mixed methods for elasticity equations is to introduce pressure as the Lagrange multiplier [12]. Our mixed method is, however, not of this type. Instead, we consider the Hellinger Reissner principle in which the primary unknowns involve both the stress tensor and the displacement. Because of the symmetry requirement for the stress tensor, it 
has been a difficult task to construct conforming mixed methods for the Hellinger Reissner formulation [39, 42].

In our mixed generalized multiscale finite element method, we build a conforming multiscale coarse space with strong-symmetry enforcement for the stress on a coarse grid. The coarse space for the displacement is chosen to be a piecewise polynomial space such that some inf-sup condition is satisfied. Following the framework of GMsFEM, we first construct the snapshot space for the stress tensor. Since we aim at conforming methods, we need to make sure the normal component of the stress tensor is continuous. To this end, we solve local pure traction problems with certain suitable compatibility conditions. The snapshot space is then further reduced by some local spectral problems. The resulting offline space consists of an edgebased space and a vertex-based space. The method is tested on various numerical examples, our experiments show robust convergence of the method against different combination of materials.

\subsection{Model problem}

Let $\Omega$ be a polygonal open subset of $\mathbb{R}^{2}$. Consider the linear elasticity system in the mixed form

$$
\begin{array}{rrr}
A \underline{\boldsymbol{\sigma}}=\varepsilon(\boldsymbol{u}) & \text { in } \quad \Omega, \\
\operatorname{div} \underline{\boldsymbol{\sigma}}=\boldsymbol{f} & \text { in } \quad \Omega, \\
\boldsymbol{u}=\mathbf{0} & \text { on } \quad \partial \Omega .
\end{array}
$$


Here, $A$ is a heterogeneous forth order tensor coefficient, $\underline{\sigma}$ is a symmetric tensor, and $\varepsilon(\boldsymbol{u})=\frac{1}{2}\left(\nabla \boldsymbol{u}+\nabla \boldsymbol{u}^{T}\right)$. Let $E$ be the Young's modulus, and $v$ be the Poisson's ratio, then the Lamé constants $\lambda$ and $\mu$ are defined to be

$$
\lambda=\frac{v E}{(1+v)(1-2 v)}, \quad \mu=\frac{E(x)}{2(1+v)} .
$$

When the material under consideration is nearly incompressible, the Poisson's ratio approaches 0.5 , and the first Lamé constant $\lambda \rightarrow \infty$. In this paper, we assume isotropic materials. In this case, if we write the stress tensor as a column vector $\sigma=\left(\sigma_{11}, \sigma_{22}, \sigma_{12}, \sigma_{21}\right)^{T}$, then the coefficient $A$ is defined to be

$$
A=\left(\begin{array}{cccc}
\lambda+2 \mu & \lambda & 0 & 0 \\
\lambda & \lambda+2 \mu & 0 & 0 \\
0 & 0 & 2 \mu & 0 \\
0 & 0 & 0 & 2 \mu
\end{array}\right)^{-1}
$$

Hence, we have

$$
\begin{aligned}
& A_{11}=A_{22}=\frac{\lambda+2 \mu}{4 \mu(\lambda+\mu)}, \\
& A_{33}=A_{44}=\frac{1}{2 \mu} .
\end{aligned}
$$

We define some bilinear forms that we will use throughout this paper. For a domain $D$,

$$
\begin{aligned}
(u, v)_{D} & =\int_{D} u v d x, \\
(\underline{\sigma}, \underline{\tau})_{A, D} & =\int_{D} A \underline{\sigma}: \underline{\tau} d x, \\
(\underline{\sigma}, \underline{\tau})_{A, \operatorname{div}, D} & =(\underline{\sigma}, \underline{\tau})_{A, D}+(\operatorname{div} \underline{\sigma}, \operatorname{div} \underline{\tau})_{D} .
\end{aligned}
$$


Their respective norms are defined to be

$$
\|u\|_{D}=(u, u)_{D}^{1 / 2},\|\underline{\sigma}\|_{A, D}=(\underline{\sigma}, \underline{\sigma})_{A, D}^{1 / 2},\|\underline{\sigma}\|_{A, \operatorname{div}, D}=(\underline{\sigma}, \underline{\sigma})_{A, \operatorname{div}, D}^{1 / 2}
$$

We will drop the subscript $D$ when $D=\Omega$. For an object $\Gamma$ of codimension 1 , we define the bilinear form

$$
<u, v>_{\Gamma}=\int_{\Gamma} u v d s
$$

The weak formulation of problem $(3.1)$ is to find $(\underline{\boldsymbol{\sigma}}, \boldsymbol{u}) \in H(\operatorname{div} ; \Omega, \mathbb{S}) \times \boldsymbol{L}^{2}(\Omega)$ such that

$$
\begin{array}{rlrl}
(A \underline{\sigma}, \underline{\tau})+(\operatorname{div} \underline{\tau}, \boldsymbol{u}) & =0 & & \forall \underline{\tau} \in H(\operatorname{div} ; \Omega, \mathbb{S}), \\
(\operatorname{div} \underline{\boldsymbol{\sigma}}, \boldsymbol{v}) & =(\boldsymbol{f}, \boldsymbol{v}) & \forall \boldsymbol{v} \in \boldsymbol{L}^{2}(\Omega) .
\end{array}
$$

Our aim is to construct finite dimensional conforming subspaces of $H(\operatorname{div} ; \Omega, \mathbb{S})$ and $\boldsymbol{L}^{2}(\Omega)$ on a coarse grid. We will simply take the coarse space for the displacement $\boldsymbol{u}$ to be piecewise polynomials on the coarse grid (without any continuity requirement). For the approximation of stress tensor $\underline{\sigma}$, we will construct two sets of multiscale basis functions, one is edge-based, the other one is vertex-based.

\subsection{Construction of the approximation space}

Let $\mathscr{T}_{H}=\bigcup_{i=1}^{N_{t}}\left\{K_{i}\right\}$ be a conforming quasi-uniform partition of $\Omega$ into rectangles, where $H$ denotes the diameter of a general element in the partition and $N_{t}$ is the number of elements. We refer to this partition as the coarse grid and assume that each coarse-grid block $K_{i}$ is partitioned into a connected union of fine-grid blocks. The fine grid partition will be denoted by $\mathscr{T}_{h}$, which by definition is a refinement of 


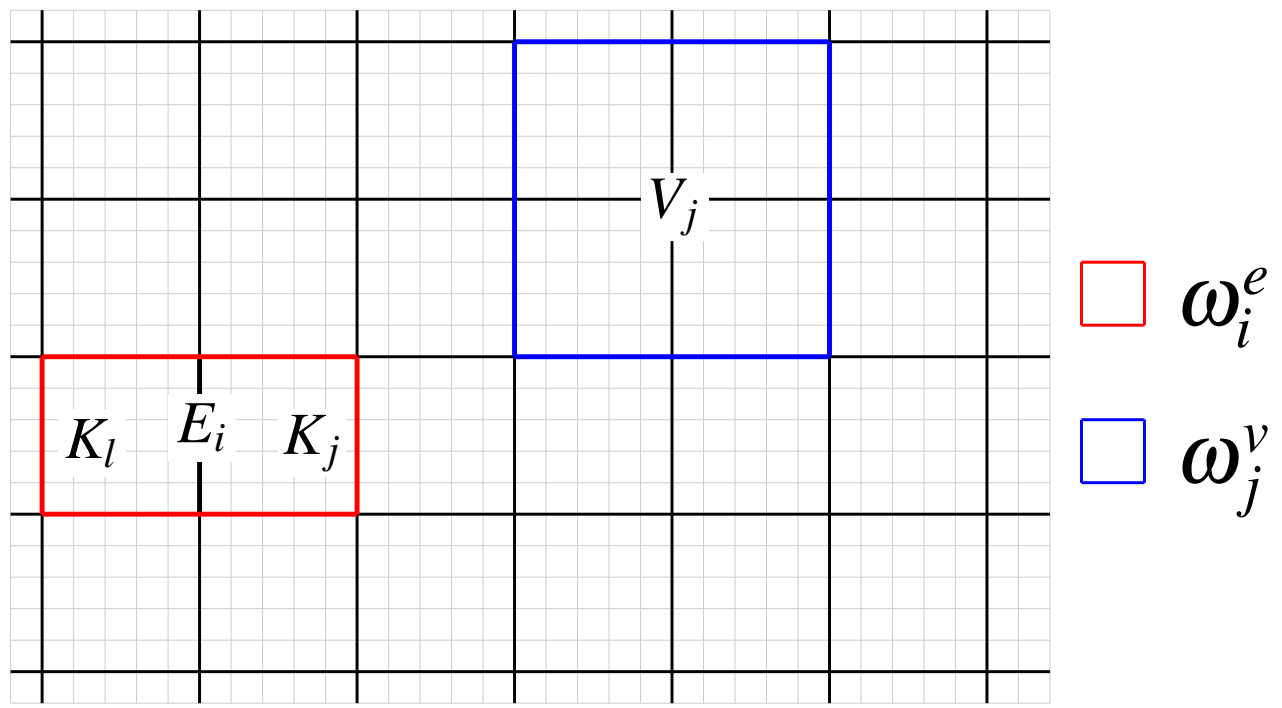

Figure 3.1: Examples of an edge-based coarse neighborhood $\omega_{i}^{e}=K_{j} \cup K_{l} \cup E_{i}$ associated with the coarse edge $E_{i}$ and a vertex-based coarse neighborhood $\omega_{j}^{v}$ associated with the coarse vertex $V_{j}$.

$\mathscr{T}_{H}$. We use $\mathscr{E}_{H}:=\bigcup_{i=1}^{N_{e}}\left\{E_{i}\right\}$ (where $N_{e}$ is the number of coarse edges) to denote the set of all edges of the coarse mesh $\mathscr{T}_{H}$. We define the coarse-edge neighborhood $\omega_{i}^{e}$ corresponding to the coarse edge $E_{i}$ as

$$
\omega_{i}^{e}=\text { interior of } \bigcup\left\{\bar{K}_{j} \in \mathscr{T}_{H} ; E_{i} \in \partial K_{j}\right\}
$$

We also define the coarse-vertex neighborhood $\omega_{i}^{v}$ corresponding to the coarse vertex $V_{i}$ as

$$
\omega_{i}^{v}=\text { interior of } \bigcup\left\{\bar{K}_{j} \in \mathscr{T}_{H} ; V_{i} \in \partial K_{j}\right\}
$$

See Figure 3.1 for examples of coarse neighborhoods, where the coarse-grid edges are denoted by solid lines and the fine-grid edges are denoted by dash lines. We discretize (3.1) by some convergent mixed finite element method on the fine grid $\mathscr{T}_{h}$ 
[39]. The fine problem is to find $\left(\underline{\sigma}_{h}, \boldsymbol{u}_{h}\right) \in \Sigma_{h} \times \boldsymbol{U}_{h}$ such that

$$
\begin{array}{rlrl}
\left(A \underline{\sigma}_{h}, \underline{\tau}_{h}\right)+\left(\operatorname{div}_{h} \underline{\tau}_{h}, \boldsymbol{u}_{h}\right) & =0 & & \underline{\tau}_{h} \in \Sigma_{h} \\
\left(\operatorname{div}_{h} \underline{\sigma}_{h}, \boldsymbol{v}_{h}\right) & =\left(\boldsymbol{f}, \boldsymbol{v}_{h}\right) & \forall \boldsymbol{v}_{h} \in \boldsymbol{U}_{h}
\end{array}
$$

We assume the fine scale pair $\left(\Sigma_{h}, \boldsymbol{U}_{h}\right)$ satisfies some inf-sup condition.

The main goal of this paper is to construct coarse spaces $\Sigma_{H}$ and $U_{H}$, which are low-dimensional subspaces of $\Sigma_{h}$ and $\boldsymbol{U}_{h}$, respectively. In order to ensure the coarse problem is well-defined, the coarse spaces need to satisfy some inf-sup stability condition. Since in mixed methods, one often is interested in approximating the stress tensor, our main focus will be on the enrichment of $\Sigma_{H}$ so that it possesses good approximation property. Once the generalized multiscale finite element space $\Sigma_{H}$ and $\boldsymbol{U}_{H}$ are constructed, the mixed generalized multiscale finite element methods for (3.1) can be stated as follows: find $\left(\underline{\boldsymbol{\sigma}}_{H}, \boldsymbol{u}_{H}\right) \in \Sigma_{H} \times \boldsymbol{U}_{H}$ such that

$$
\begin{aligned}
\left(A \underline{\sigma}_{H}, \underline{\tau}_{H}\right)+\left(\operatorname{div}_{h} \underline{\tau}_{H}, \boldsymbol{u}_{H}\right) & =0 & \forall \underline{\tau}_{H} \in \Sigma_{H} \\
\left(\operatorname{div}_{h} \underline{\sigma}_{H}, \boldsymbol{v}_{H}\right) & =\left(\boldsymbol{f}, \boldsymbol{v}_{H}\right) & \forall \boldsymbol{v}_{H} \in \boldsymbol{U}_{H}
\end{aligned}
$$

Following the general framework suggested by GMsFEM, we will construct a multiscale finite element space $\Sigma_{H}$ for the approximation of the stress tensor. The dimension of $\Sigma_{H}$ is flexible and can be chosen by the user. In our construction, $\Sigma_{H}$ is the union of a edge-based space $\Sigma_{\text {off }}^{V}$ and a vertex-based space $\Sigma_{\text {off }}^{V}$, i.e.

$$
\Sigma_{H}=\Sigma_{\mathrm{off}}^{E} \cup \Sigma_{\mathrm{off}}^{V}
$$

We will describe the detailed construction of $\Sigma_{\text {off }}^{E}$ and $\Sigma_{\text {of }}^{V}$ in the following section. 


\subsubsection{Edge-based basis functions for the stress tensor}

In this section, we will construct edge-based $H(\operatorname{div} ; \Omega, \mathbb{S})$ conforming basis functions for the stress tensor from some local problems. Consider the coarse neighborhood $\omega_{i}$ associated with a coarse edge $E_{i}$. Notice that $E_{i}$ can be written as a union of fine-grid edges, namely, $E_{i}=\cup_{S=1}^{S_{i}} e_{s}$, where $S^{E_{i}}$ is the total number of fine-grid edges on $E_{i}$ and $e_{s}$ denote a fine grid edge. Let $\delta_{s}^{E_{i}}$ be a piecewise constant function defined on $E_{i}$ with respect to the fine-grid such that it has value $1 /\left|e_{s}\right|$ on $e_{s}$ and value 0 on the other fine-grid edges; that is,

$$
\delta_{s}^{E_{i}}=\left\{\begin{array}{ll}
1, & \text { on } e_{s}, \\
0, & \text { on } E_{i} \backslash e_{s},
\end{array} \quad s=1,2, \cdots, S^{E_{i}} .\right.
$$

We are going to solve some local pure traction problems. It is well-known that certain compatibility conditions need to be satisfied so that a pure traction problem has a solution. The compatibility conditions will involve the space of rigid motion

$$
R M=\operatorname{span}\left\{\left(\begin{array}{l}
1 \\
0
\end{array}\right),\left(\begin{array}{l}
0 \\
1
\end{array}\right),\left(\begin{array}{c}
x_{2} \\
-x_{1}
\end{array}\right)\right\}
$$

We will discuss the case when $E_{i}$ is an interior coarse edge, the case when $E_{i}$ is a boundary edge can be treat similarly (in fact, the construction in the latter case is even simpler since there is only one coarse block sharing $E_{i}$ ). If $E_{i}$ is located in the interior of $\Omega$, then there exist coarse blocks $K_{l}$ and $K_{j}$ such that $E_{i}=K_{l} \cap K_{j}$. On $\omega_{i}^{e}=K_{l} \cup K_{j} \cup E_{i}$, we will obtain a set of local snapshot basis functions $\left\{\underline{\phi}_{s}^{E_{i}}\right\}$ by 
solving the local pure traction problems

$$
\begin{aligned}
& A \underline{\phi}_{s}^{E_{i}}=\varepsilon\left(\boldsymbol{\varphi}_{s}^{E_{i}}\right) \quad \text { in } \quad K_{l}, K_{j} \\
& \operatorname{div} \underline{\phi}_{s}^{E_{i}}=\boldsymbol{f}_{s}^{E_{i}} \quad \text { in } \quad K_{l}, K_{j} \\
& \underline{\phi}_{s}^{E_{i}} \cdot \boldsymbol{n}=0 \quad \text { on } \quad \partial \omega_{i}^{e} \\
& \underline{\phi}_{s}^{E_{i}} \cdot \boldsymbol{m}_{i}=\boldsymbol{b}_{s}^{E_{i}} \quad \text { on } \quad E_{i} .
\end{aligned}
$$

Here, $\boldsymbol{n}$ is the unit outward normal of $\omega_{i}$, and $\boldsymbol{m}_{i}$ is a unit outward normal of $K_{l}$ (a unit inward normal of $\left.K_{j}\right)$. If $E_{i}$ is a vertical edge, then $\boldsymbol{b}_{s}^{E_{i}}=\left(\delta_{s}^{E_{i}}, 0\right)^{T}$. On the other hand, if $E_{i}$ is a horizontal edge, then $\boldsymbol{b}_{s}^{E_{i}}=\left(0, \delta_{s}^{E_{i}}\right)^{T}$. The right hand side function $\boldsymbol{f}_{s}^{E_{i}}$ is defined piecewisely on the coarse blocks $K_{l}$ and $K_{j}$. More precisely, we take the restriction of $\boldsymbol{f}_{s}^{E_{i}}$ to $K_{l}$ to be a function in $R M$ (i.e. $\left.\boldsymbol{f}_{s}^{E_{i}}\right|_{K_{l}} \in R M$ ), such that

$$
\left(\left.\boldsymbol{f}_{s}^{E_{i}}\right|_{K_{l}}, \boldsymbol{v}\right)_{K_{l}}+<\underline{\phi}_{s}^{E_{i}} \cdot \boldsymbol{n}_{l}, \boldsymbol{v}>_{\partial K_{l}}=0 \quad \forall \boldsymbol{v} \in R M
$$

where $\boldsymbol{n}_{l}$ is the unit outward normal of $K_{l}$. It is obvious that compatibility condition (3.7) uniquely defines $\left.\boldsymbol{f}_{s}^{E_{i}}\right|_{K_{l}} \in R M$. Notice that $\underline{\phi}_{s}^{E_{i}} \cdot \boldsymbol{n}_{l}$ on $\partial K_{l}$ is given, so $\left.\boldsymbol{f}_{s}^{E_{i}}\right|_{K_{l}}$ can be computed before we solve (3.6). We can define the restriction of $\boldsymbol{f}_{s}^{E_{i}}$ to $K_{j}$ in a similar manner. With the compatibility condition (3.7), the solution $\underline{\phi}_{s}^{E_{i}}$ of (3.6) is uniquely determined, c.f. [9, lemma 2.3].

Remark 3.2.1 In fact, [9, lemma 2.3] states that the solution $\boldsymbol{\varphi}_{s}^{E_{i}}$ of (3.6) is uniquely determined up to a function in the space of rigid motion $R M$. But since

$$
\varepsilon(\boldsymbol{v})=0 \quad \forall \boldsymbol{v} \in R M,
$$

we can conclude that $\underline{\phi}_{s}^{E_{i}}$ is unique. 
Let

$$
\Sigma_{\text {snap }}^{E_{i}}=\operatorname{span}\left\{\underline{\phi}_{s}^{E_{i}}: s=1,2, \cdots, S^{E_{i}}\right\}
$$

We will construct a low-dimensional subspace of $\Sigma_{\text {snap. }}^{E_{i}}$ For this purpose, we define the bilinear forms

$$
c_{i}(\underline{\sigma}, \underline{\tau})=<A_{11} \underline{\sigma} \cdot \boldsymbol{m}_{i}, \underline{\tau} \cdot \boldsymbol{m}_{i}>_{E_{i}},
$$

where $A_{11}$ is defined in (3.2) We then solve the eigenvalue problem: find $\left(\underline{\psi}_{l}^{E_{i}}, \lambda_{l}^{E_{i}}\right) \in$ $\Sigma_{\text {snap }}^{E_{i}} \times \mathbb{R}$ such that

$$
c_{i}\left(\underline{\psi}_{l}^{E_{i}}, \underline{\phi}^{E_{i}}\right)=\lambda_{l}^{E_{i}}\left(\underline{\psi}_{l}^{E_{i}}, \underline{\phi}^{E_{i}}\right)_{A, \operatorname{div}, \omega_{i}^{e}} \forall \underline{\phi}^{E_{i}} \in \Sigma_{\mathrm{Snap}}^{E_{i}}
$$

We order the eigenvalue in ascending order

$$
\lambda_{1}^{E_{i}} \leq \lambda_{2}^{E_{i}} \leq \cdots \leq \lambda_{S^{E_{i}}}^{E_{i}}
$$

Then we collect the eigenfunctions corresponding to the smallest $L^{E_{i}}$ eigenvalues which span our local offline space, i.e.

$$
\Sigma_{\text {off }}^{E_{i}}=\operatorname{span}\left\{\underline{\psi}_{l}^{E_{i}}: \imath=1,2, \cdots, L^{E_{i}}\right\}
$$

In general, $L^{E_{i}}<<S^{E_{i}}$. Lastly, the offline space of all edge-based multiscale basis functions for the stress tensor is defined to be the direct sum of all the local offline spaces

$$
\Sigma_{\mathrm{off}}^{E}=\bigoplus_{E_{i} \in \mathscr{E}_{H}} \Sigma_{\mathrm{off}}^{E_{i}} .
$$




\subsubsection{Vertex-based basis functions for the stress tensor}

Consider a coarse vertex $V_{i}$ and the coarse-vertex neighborhood $\omega_{i}^{v}$. There may exist 2,3 , or 4 coarse vertices $V_{k}$ (depending on the location of $V_{i}$ ) such that $V_{k}$ 's are connected to $V_{i}$ by coarse edges $E_{k}$ 's. Let

$$
\Xi^{V_{i}}=\bigcup\left(E_{k} \backslash\left\{V_{k}\right\}\right)
$$

Suppose $x_{s}, s=1,2, \cdots, S^{V_{i}}$ are fine-grid nodes on $\Xi^{V_{i}}$, where $S^{V_{i}}$ is the total number of fine-grid nodes on $\Xi^{V_{i}}$. We define $\eta_{s}^{V_{i}}$ to be a piecewise linear (on each fine-grid edge) function on $\Xi^{V_{i}}$ such that

$$
\eta_{s}^{V_{i}}=\left\{\begin{array}{ll}
1, & \text { at } x_{s}, \\
0, & \text { at other nodes on } \Xi^{V_{i},}
\end{array} \quad s=1,2, \cdots, S^{V_{i}} .\right.
$$

We will discuss the case when $V_{i}$ is an interior coarse vertex, the treatment for boundary coarse vertices should be a straight forward modification to the treatment for interior vertices. Now suppose $V_{i}$ is an interior coarse vertex. We then solve the local boundary value problems

$$
\begin{array}{rlrl}
A \underline{\phi}_{s}^{V_{i}} & =\boldsymbol{\varepsilon}\left(\boldsymbol{\varphi}_{s}^{V_{i}}\right) & \text { in all } & K_{l} \subseteq \omega_{i}^{v} \\
\operatorname{div} \underline{\phi}_{s}^{V_{i}}=\boldsymbol{f}_{s}^{V_{i}} & \text { in all } & K_{l} \subseteq \omega_{i}^{v} \\
\underline{\phi}_{s}^{V_{i}} \cdot \boldsymbol{n} & =0 & \text { on } \partial \omega_{i}^{v} \\
\underline{\phi}_{s}^{V_{i}} \cdot \boldsymbol{m} & =\boldsymbol{b}_{s}^{V_{i}} & \text { on } \Xi^{V_{i}}
\end{array}
$$

Here, $\boldsymbol{n}$ is the unit outward normal to $\partial \omega_{i}^{v}$ and $\boldsymbol{m}$ is a fixed unit normal from one coarse block to another. If $x_{s}$ is located at a vertical coarse edge, then $\boldsymbol{b}_{s}^{V_{i}}=\left(0, \eta_{s}^{V_{i}}\right)^{T}$. On the other hand, if $x_{s}$ is located at a horizontal coarse edge, then $\boldsymbol{b}_{s}^{V_{i}}=\left(\eta_{s}^{V_{i}}, 0\right)^{T}$. 
Lastly, if $x_{s}$ is located at $V_{i}$, then $\boldsymbol{b}_{s}^{V_{i}}=\left(\eta_{s}^{V_{i}}, \eta_{s}^{V_{i}}\right)^{T}$. Similar to the edge-based case, $f_{s}^{V_{i}}$ is defined piecewisely on each $K_{l} \subseteq \omega_{i}^{v}$ such that it satisfies the compatibility condition (3.7), which guarantees the existence and uniqueness of $\underline{\phi}_{s}^{V_{i}}$. Let

$$
\Sigma_{\text {snap }}^{V_{i}}=\operatorname{span}\left\{\underline{\phi}_{s}^{V_{i}}: s=1,2, \cdots, S^{V_{i}}\right\} .
$$

Again, we will reduce the dimension of this local space. We define

$$
d_{i}(\underline{\sigma}, \underline{\tau})=<A_{33} \underline{\sigma} \cdot \boldsymbol{m}, \underline{\tau} \cdot \boldsymbol{m}>_{\Xi^{V}} V_{i}
$$

where $A_{33}$ is defined in (3.2). We solve the eigenvalue problem: find $\left(\underline{\psi}_{l}^{V_{i}}, \lambda_{l}^{V_{i}}\right) \in$ $\Sigma_{\text {snap }}^{V_{i}} \times \mathbb{R}$ such that

$$
d_{i}\left(\underline{\psi}_{l}^{V_{i}}, \underline{\phi}^{V_{i}}\right)=\lambda_{l}^{V_{i}}\left(\underline{\psi}_{l}^{V_{i}}, \underline{\phi}^{V_{i}}\right)_{A, \operatorname{div}, \omega_{i}^{v}} \forall \underline{\phi}^{V_{i}} \in \Sigma_{\text {snap }}^{V_{i}} .
$$

We order the eigenvalue in ascending order

$$
\lambda_{1}^{V_{i}} \leq \lambda_{2}^{V_{i}} \leq \cdots \leq \lambda_{S_{i}^{V_{i}}}^{V_{i}}
$$

Then we collect the eigenfunctions corresponding to the smallest $L^{V_{i}}$ eigenvalues which span our local offline space, i.e.

$$
\Sigma_{\text {off }}^{V_{i}}=\operatorname{span}\left\{\underline{\psi}_{l}^{V_{i}}: \imath=1,2, \cdots, L^{V_{i}}\right\} .
$$

The vertex-based space is

$$
\Sigma_{\mathrm{off}}^{V}=\bigoplus_{V_{i}} \Sigma_{\mathrm{off}}^{V_{i}}
$$




\subsubsection{Coarse space for displacement}

In order to ensure inf-sup stability of the saddle point problem (3.5), the coarse space $\boldsymbol{U}_{H}$ for the displacement $\boldsymbol{u}$ is taken such that

$$
\operatorname{div} \Sigma_{H}=\boldsymbol{U}_{H}
$$

Since by construction, the divergence of all the basis functions in $\Sigma_{H}$ are in the space of rigid motion $R M$, the obvious choice for $\boldsymbol{U}_{H}$ is

$$
\boldsymbol{U}_{H}=\left\{\boldsymbol{v}_{H} \in \boldsymbol{U}_{h}:\left.\boldsymbol{v}_{H}\right|_{K_{j}} \in R M, \forall K_{j} \in \mathscr{T}_{H}\right\} .
$$

\subsection{Stability and convergence}

Some error estimate will be derived for the stress tensor in this section. We begin by defining several projections, which can help analyzing the errors due to the snapshot and offline spaces. Then an inf-sup condition for the coarse space pair $\left(\Sigma_{H}, U_{H}\right)$ will be discussed. Lastly, we will show that the error of the coarse stress solution is bounded by some projection errors.

\subsubsection{Snapshot projection}

Recall that $\underline{\sigma}_{h}$ and $\boldsymbol{u}_{h}$ are the solutions to the fine problem (3.4). Let $\Pi_{H}^{U}$ : $\boldsymbol{L}^{2}(\Omega) \rightarrow \boldsymbol{U}_{H}$ be the usual $\boldsymbol{L}^{2}$ projection from $\boldsymbol{L}^{2}(\Omega)$ to the coarse space $\boldsymbol{U}_{H}$. That is, for any $\boldsymbol{f} \in \boldsymbol{L}^{2}(\Omega)$, we have $\Pi_{H}^{U} \boldsymbol{f} \in \boldsymbol{U}_{H}$ and

$$
\left(\Pi_{H}^{U} \boldsymbol{f}, \boldsymbol{v}_{H}\right)=\left(\boldsymbol{f}, \boldsymbol{v}_{H}\right) \forall \boldsymbol{v}_{H} \in \boldsymbol{U}_{H}
$$


For the fine solution $\underline{\sigma}_{h} \in \Sigma_{h}$, define its snapshot projection $\underline{\widehat{\sigma}}_{h} \in \Sigma_{h}$ to be a function such that for $K_{j} \in \mathscr{T}_{H}$,

$$
\begin{aligned}
& A \underline{\widehat{\sigma}}_{h}=\varepsilon\left(\widehat{\boldsymbol{u}}_{h}\right) \quad \text { in } \quad K_{j}, \\
& \operatorname{div}_{h} \underline{\widehat{\sigma}}_{h}=\Pi_{H}^{U} \boldsymbol{f} \quad \text { in } \quad K_{j}, \\
& \underline{\widehat{\sigma}}_{h} \cdot \boldsymbol{n}_{j}=\underline{\boldsymbol{\sigma}}_{h} \cdot \boldsymbol{n}_{j} \quad \text { on } \quad \partial K_{j}, \\
& \left(\widehat{\boldsymbol{u}}_{h}-\boldsymbol{u}_{h}, \boldsymbol{v}\right)_{K_{j}}=0 \quad \forall \boldsymbol{v} \in R M .
\end{aligned}
$$

Note that

$$
(\boldsymbol{f}, \boldsymbol{v})_{K_{j}}+<\underline{\sigma}_{h} \cdot n_{j}, \boldsymbol{v}>_{\partial K_{j}}=0 \quad \forall \boldsymbol{v} \in R M
$$

This and (3.13) imply

$$
\left(\Pi_{H}^{U} f, \boldsymbol{v}\right)_{K_{j}}+<\underline{\hat{\sigma}}_{h} \cdot \boldsymbol{n}_{j}, \boldsymbol{v}>_{\partial K_{j}}=0 \quad \forall \boldsymbol{v} \in R M .
$$

So the local pure traction problem (3.14) is well defined and admits a unique solution $\widehat{\widehat{\sigma}}_{h}$. In fact, the above snapshot projection can be defined for any fine grid function (we call it snapshot projection because the range of the projection is in the snapshot space). The following proposition shows that the error between the fine grid stress solution and its snapshot projection is bounded by the $\boldsymbol{L}^{2}$ projection error induced when projecting the source term $\boldsymbol{f}$ onto the coarse displacement space $\boldsymbol{U}_{H}$.

Proposition 3.3.1 Let $\underline{\sigma}_{h} \in \Sigma_{h}$ be the fine grid stress solution and $\underline{\widehat{\sigma}}_{h}$ be its snapshot projection defined in (3.14), then

$$
\left\|\underline{\widehat{\sigma}}_{h}-\underline{\sigma}_{h}\right\|_{A, K_{j}} \leq C(A)\left\|\boldsymbol{f}-\Pi_{H}^{U} \boldsymbol{f}\right\|_{K_{j}}
$$

where the constant $C(A)$ depends on the coefficient $A$ but not on $H$. 
Proof. Subtracting (3.4) from the weak form of (3.14), we have

$$
\begin{array}{rlr}
\left(A \underline{\widehat{\sigma}}_{h}-A \underline{\boldsymbol{\sigma}}_{h}, \underline{\tau}_{h}\right)_{K_{j}} & =\left(\operatorname{div} \underline{\tau}_{h}, \boldsymbol{u}_{h}-\widehat{\boldsymbol{u}}_{h}\right)_{K_{j}} & \forall \underline{\tau}_{h} \in \Sigma_{h, 0}\left(K_{j}\right), \\
\left(\operatorname{div}_{h} \widehat{\widehat{\sigma}}_{h}-\operatorname{div}_{h} \underline{\boldsymbol{\sigma}}_{h}, \boldsymbol{v}_{h}\right)_{K_{j}}=\left(\Pi_{H}^{U} \boldsymbol{f}-\boldsymbol{f}, \boldsymbol{v}_{h}\right)_{K_{j}} & \forall \boldsymbol{v}_{h} \in \boldsymbol{U}_{h}\left(K_{j}\right) .
\end{array}
$$

Here, $\Sigma_{h, 0}\left(K_{j}\right)$ is the restriction of $\Sigma_{h}$ only to the interior of $K_{j}$, and $\boldsymbol{U}_{h}\left(K_{j}\right)$ is the restriction of $\boldsymbol{U}_{h}$ to $K_{j}$. Since $\underline{\widehat{\sigma}}_{h} \cdot \boldsymbol{n}_{j}=\underline{\sigma}_{h} \cdot \boldsymbol{n}_{j}$ on $\partial K_{j}, \underline{\widehat{\sigma}}_{h}-\underline{\sigma}_{h}$ belongs to $\Sigma_{h, 0}\left(K_{j}\right)$. Thus, we can take $\underline{\tau}_{h}=\underline{\widehat{\sigma}}_{h}-\underline{\sigma}_{h}$ and $\boldsymbol{v}_{h}=\widehat{\boldsymbol{u}}_{h}-\boldsymbol{u}_{h}$ in (3.16), then we get

$$
\left(A \underline{\widehat{\sigma}}_{h}-A \underline{\sigma}_{h}, \underline{\hat{\sigma}}_{h}-\underline{\sigma}_{h}\right)_{K_{j}}=\left(\boldsymbol{f}-\Pi_{H}^{U} \boldsymbol{f}, \widehat{\boldsymbol{u}}_{h}-\boldsymbol{u}_{h}\right)_{K_{j}} .
$$

Because of the condition $\left(\widehat{\boldsymbol{u}}_{h}-\boldsymbol{u}_{h}, \boldsymbol{v}\right)_{K_{j}}=0, \forall \boldsymbol{v} \in R M$, the inf-sup stability condition for the pair $\left(\Sigma_{h, 0}\left(K_{j}\right), \boldsymbol{U}_{h}\left(K_{j}\right)\right)$ can be applied. So we have

$$
\begin{aligned}
\left\|\boldsymbol{u}_{h}-\widehat{\boldsymbol{u}}_{h}\right\|_{K_{j}} & \leq C \sup _{\underline{\tau}_{h} \in \Sigma_{h, 0}\left(K_{j}\right)} \frac{\left(\operatorname{div}_{h} \underline{\tau}_{h}, \boldsymbol{u}_{h}-\widehat{\boldsymbol{u}}_{h}\right)_{K_{j}}}{\left\|\underline{\tau}_{h}\right\|_{H\left(\operatorname{div} ; K_{j}\right)}} \\
& \leq C(A) \sup _{\underline{\tau}_{h} \in \Sigma_{h, 0}\left(K_{j}\right)} \frac{\left(A \underline{\widehat{\sigma}}_{h}-A \underline{\sigma}_{h}, \underline{\tau}_{h}\right)_{K_{j}}}{\left\|\underline{\tau}_{h}\right\|_{A, K_{j}}} \\
& \leq C(A)\left\|\underline{\widehat{\sigma}}_{h}-\underline{\sigma}_{h}\right\|_{A, K_{j}} .
\end{aligned}
$$

Hence, from (3.17) and (3.18), we deduce that the error bound for the snapshot projection.

\subsubsection{The projection $\Pi_{H}^{\Sigma}$}

Next, we will define another projection $\Pi_{H}^{\Sigma}$ which projects the snapshot solution $\widehat{\widehat{\sigma}}_{h}$ onto $\Sigma_{H}$. To this end, we need some notations. On a coarse edge $E_{i}$ with unit normal $\boldsymbol{n}_{i}$, we let $\widehat{\sigma}_{h}^{n, 1}$ and $\widehat{\sigma}_{h}^{n, 2}$ be the first and second components of the normal 
component of $\underline{\widehat{\sigma}}_{h}$, i.e.

$$
\underline{\underline{\sigma}}_{h} \cdot \boldsymbol{n}_{i}=\left(\begin{array}{c}
\widehat{\sigma}_{h}^{n, 1} \\
\hat{\sigma}_{h}^{n, 2}
\end{array}\right)
$$

Furthermore, since $\mathscr{T}_{H}$ is a rectangular grid, we can split edges into horizontal edges $\mathscr{E}_{H} h o r$ and vertical edges $\mathscr{E}_{H}^{v e r}$. Let $\underline{\widehat{\sigma}}_{h}^{e}$ be defined piecewisely such that in each $K_{j} \in \mathscr{T}_{H}$,

$$
\begin{array}{rr}
A \underline{\widehat{\sigma}}_{h}^{e}=\varepsilon\left(\widehat{\boldsymbol{u}}_{h}^{e}\right) & \text { in } K_{j}, \\
\operatorname{div} \underline{\widehat{\sigma}}_{h}^{e}=\widehat{f}^{e} & \text { in } K_{j}, \\
\underline{\widehat{\boldsymbol{\sigma}}}_{h}^{e} \cdot \boldsymbol{n}_{j}=\left(\begin{array}{c}
\widehat{\boldsymbol{\sigma}}_{h}^{n, 1} \\
0
\end{array}\right) & \text { on } \partial K_{j} \cap \mathscr{E}_{H} \mathrm{ver}, \\
\underline{\widehat{\boldsymbol{\sigma}}}_{h}^{e} \cdot \boldsymbol{n}_{j}=\left(\begin{array}{c}
0 \\
\hat{\boldsymbol{\sigma}}_{h}^{n, 2}
\end{array}\right) & \text { on } \partial K_{j} \cap \mathscr{E}_{H},
\end{array}
$$

where $\left.\widehat{\boldsymbol{f}}^{e}\right|_{K_{j}}$ is a function in $R M$ such that the following compatibility condition is satisfied

$$
\left(\widehat{\boldsymbol{f}}^{e}, \boldsymbol{v}\right)_{K_{j}}+<{\underline{\widehat{\sigma}_{h}^{e}}}_{h} \cdot \boldsymbol{n}_{j}, \boldsymbol{v}>_{\partial K_{j}}=0 \quad \forall \boldsymbol{v} \in R M .
$$

Similarly, let $\underline{\widehat{\sigma}}_{h}^{v}$ be defined piecewisely such that in each $K_{j} \in \mathscr{T}_{H}$,

$$
\begin{array}{rr}
A \underline{\widehat{\sigma}}_{h}^{v}=\varepsilon\left(\widehat{\boldsymbol{u}}_{h}^{v}\right) & \text { in } K_{j}, \\
\operatorname{div} \underline{\widehat{\boldsymbol{\sigma}}}_{h}^{v}=\widehat{\boldsymbol{f}}^{v} & \text { in } K_{j}, \\
\underline{\widehat{\boldsymbol{\sigma}}}_{h}^{v} \cdot \boldsymbol{n}_{j}=\left(\begin{array}{c}
0 \\
\hat{\boldsymbol{\sigma}}_{h}^{n, 2}
\end{array}\right) & \text { on } \partial K_{j} \cap \mathscr{E}_{H}^{v e r}, \\
\underline{\widehat{\boldsymbol{\sigma}}}_{h}^{v} \cdot \boldsymbol{n}_{j}=\left(\begin{array}{c}
\widehat{\boldsymbol{\sigma}}_{h}^{n, 1} \\
0
\end{array}\right) & \text { on } \partial K_{j} \cap \mathscr{E}_{H},
\end{array}
$$


where $\left.\widehat{\boldsymbol{f}}^{v}\right|_{K_{j}}$ is a function in $R M$ such that the following compatibility condition is satisfied

$$
\left(\widehat{f}^{v}, \boldsymbol{v}\right)_{K_{j}}+<\underline{\widehat{\sigma}}_{h}^{v} \cdot \boldsymbol{n}_{j}, \boldsymbol{v}>_{\partial K_{j}}=0 \quad \forall \boldsymbol{v} \in R M
$$

Notice that $\forall \boldsymbol{v} \in R M$,

$$
\begin{aligned}
\left(\widehat{\boldsymbol{f}}^{e}+\widehat{\boldsymbol{f}}^{v}, \boldsymbol{v}\right)_{K_{j}} & =-<\underline{\widehat{\sigma}}_{h}^{e} \cdot \boldsymbol{n}_{j}+\underline{\widehat{\boldsymbol{\sigma}}}_{h}^{v} \cdot \boldsymbol{n}_{j}, \boldsymbol{v}>_{\partial K_{j}} \\
& =-<\underline{\hat{\sigma}}_{h} \cdot \boldsymbol{n}_{j}, \boldsymbol{v}>_{\partial K_{j}} \\
& =\left(\Pi_{H}^{U} \boldsymbol{f}, \boldsymbol{v}\right)_{K_{j}} .
\end{aligned}
$$

Since $\widehat{\boldsymbol{f}}^{e}, \widehat{\boldsymbol{f}}^{v}$ and $\Pi_{H}^{U} \boldsymbol{f}$ are functions in $\boldsymbol{U}_{H}$, we actually have

$$
\widehat{\boldsymbol{f}}^{e}+\widehat{\boldsymbol{f}}^{v}=\Pi_{H}^{U} \boldsymbol{f}
$$

Hence, by the principal of superposition and the uniqueness of solution to the local pure traction problems, we can see that

$$
\underline{\widehat{\sigma}}_{h}=\underline{\hat{\sigma}}_{h}^{e}+\underline{\hat{\sigma}}_{h}^{v}
$$

We observe that $\underline{\hat{\sigma}}_{h}^{e} \in \bigoplus_{E_{i}} \Sigma_{\text {snap }}^{E_{i}}$ and $\underline{\hat{\sigma}}_{h}^{v} \in \bigoplus_{V_{i}} \Sigma_{\text {snap }}^{V_{i}}$. So we will approximate $\underline{\hat{\sigma}}_{h}^{e}$ by the edge-based space $\Sigma_{\text {off }}^{E}$, and approximate $\underline{\widehat{\sigma}}_{h}^{v}$ by the vertex-based space $\Sigma_{\text {off }}^{V}$. Since the local snapshot spaces $\Sigma_{\text {snap }}^{E_{i}}$ are mutually disjoint, we can write $\underline{\widehat{\sigma}}_{h}^{e}=\sum_{i} \underline{\widehat{\sigma}}_{h}^{e, i}$ with each $\underline{\widehat{\sigma}}_{h}^{e, i} \in \Sigma_{\text {snap }}^{E_{i}}$. Thus, each $\underline{\widehat{\sigma}}_{h}^{e, i}$ can be written as a linear combination of the local eigenvectors in (3.8). That is,

$$
\underline{\widehat{\sigma}}_{h}^{e, i}=\sum_{l=1}^{S_{i}} \alpha_{l}^{i} \underline{\psi}_{l}^{E_{i}}
$$

Then, we define a local projection $I_{H}^{E_{i}}: \Sigma_{\text {snap }}^{E_{i}} \rightarrow \Sigma_{\text {off }}^{E_{i}}$ such that

$$
I_{H}^{E_{i}}\left(\underline{\widehat{\sigma}}_{h}^{e, i}\right)=\sum_{l=1}^{L^{E_{i}}} \alpha_{l}^{i} \underline{\psi}_{l}^{E_{i}} .
$$


Because $\underline{\psi}_{l}^{E_{i}}$ are eigenvectors of (3.8), and the corresponding eigenvalues $\lambda_{l}^{E_{i}}$ are order as in (3.9), we deduce that

$$
\left\|\underline{\widehat{\sigma}}_{h}^{e, i}-I_{H}^{E_{i}}\left(\underline{\widehat{\sigma}}_{h}^{e, i}\right)\right\|_{A, \operatorname{div}, \omega_{i}^{e}}^{2} \leq \frac{1}{\lambda_{L^{E_{i}+1}}^{E_{i}}} c_{i}\left(\underline{\widehat{\sigma}}_{h}^{e, i}, \underline{\widehat{\sigma}}_{h}^{e, i}\right)=\frac{1}{\lambda_{L^{E_{i}+1}}^{E_{i}}} c_{i}\left(\underline{\widehat{\sigma}}_{h}^{e}, \underline{\widehat{\sigma}}_{h}^{e}\right) .
$$

Let $\Pi_{H}^{\Sigma, E}\left(\underline{\widehat{\sigma}}_{h}^{e}\right)=\sum_{i} I_{H}^{E_{i}}\left(\underline{\hat{\sigma}}_{h}^{e, i}\right)$. It is obvious that $\Pi_{H}^{\Sigma, E}\left(\underline{\widehat{\sigma}}_{h}^{e}\right) \in \Sigma_{\text {off }}^{E}$. Moreover,

$$
\left\|\underline{\hat{\sigma}}_{h}^{e}-\Pi_{H}^{\Sigma, E}\left(\underline{\hat{\sigma}}_{h}^{e}\right)\right\|_{A, \operatorname{div}}^{2} \leq \frac{1}{\Lambda^{E}} \sum_{i} c_{i}\left(\underline{\hat{\sigma}}_{h}^{e}, \underline{\hat{\sigma}}_{h}^{e}\right),
$$

where $\Lambda^{E}=\min _{E_{i}}\left(\lambda_{L^{E_{i}+1}}^{E_{i}}\right)$.

The treatment for $\underline{\hat{\sigma}}_{h}^{v}$ is slightly different. This is due to the fact that the pairwise intersection of the local snapshot spaces $\Sigma_{\text {snap }}^{V_{i}}$ may not be empty. Hence, we need a partition of unity for the decomposition of $\underline{\widehat{\sigma}}_{h}^{v}$. Let $\left\{\chi_{i}\right\} \subseteq C(\Omega)$ be a partition of unity for the domain $\Omega$ such that

$$
\operatorname{supp}\left(\chi_{i}\right) \subseteq \omega_{i}^{v} \text { and } \sum_{i} \chi_{i} \equiv 1
$$

Then we can write $\underline{\hat{\sigma}}_{h}^{v}=\sum_{i}\left(\underline{\hat{\sigma}}_{h}^{v} \chi_{i}\right)$ with each $\underline{\hat{\sigma}}_{h}^{v} \chi_{i}$ being supported in $\omega_{i}^{v}$. However, $\underline{\widehat{\sigma}}_{h}^{v} \chi_{i}$ may not belong to $\Sigma_{\text {snap }}^{V_{i}}$ since its divergence may not be in the space of rigid motion $R M$. To overcome this problem, we first define a $L^{2}$ projection $\pi_{h}:\left(L^{2}(\Omega)\right)^{2 \times 2} \rightarrow \Sigma_{h}$ such that for $\underline{\sigma} \in\left(L^{2}(\Omega)\right)^{2 \times 2}$,

$$
\left(\pi_{h} \underline{\sigma}, \underline{\tau}_{h}\right)=\left(\underline{\sigma}, \underline{\tau}_{h}\right) \forall \underline{\tau}_{u} \in \Sigma_{h}
$$

Then, $\pi_{h}\left(\widehat{\sigma}_{h}^{v} \chi_{i}\right) \in \Sigma_{h}$ is a fine grid function, so we can define $\left.\widehat{\pi_{h}\left(\widehat{\widehat{\sigma}}_{h}^{v} \chi_{i}\right.}\right)$ in the same way as we define $\underline{\widehat{\sigma}}_{h}$ from $\underline{\sigma}_{h}$, see (3.14). Then $\bar{\pi}_{h\left(\underline{\widehat{\sigma}}_{h}^{v} \chi_{i}\right)} \in \Sigma_{\text {snap }}^{V_{i}}$ and

$$
\sum_{i} \pi_{h\left(\hat{\widehat{\sigma}}_{h}^{v} \chi_{i}\right)}=\widehat{\pi_{h}\left(\hat{\underline{\sigma}}_{h}^{v}\right)}=\underline{\underline{\sigma}}_{h}^{v}
$$

Now, by making use of spectral problem (3.11), we can define local projections from 
each $\Sigma_{\text {snap }}^{V_{i}}$ to the corresponding $\Sigma_{\text {off }}^{V_{i}}$. The rest of the steps are similar to the treatment of $\underline{\hat{\sigma}}_{h}^{e}$, so we omit the detail here. In short, we can define $\Pi_{H}^{\Sigma, V}\left(\underline{\hat{\sigma}}_{h}^{v}\right) \in \Sigma_{\text {off }}^{V}$ such that

$$
\left\|\underline{\underline{\sigma}}_{h}^{v}-\Pi_{H}^{\Sigma, V}\left(\underline{\hat{\sigma}}_{h}^{v}\right)\right\|_{A, \operatorname{div}}^{2} \leq \frac{1}{\Lambda^{V}} \sum_{i} d_{i}\left(\underline{\widehat{\sigma}}_{h}^{v}, \underline{\hat{\sigma}}_{h}^{v}\right) .
$$

where $\Lambda^{V}=\min _{V_{i}}\left(\lambda_{L^{V_{i+1}}}^{V_{i}}\right)$. Lastly, the interpolant $\Pi_{H}^{\Sigma}\left(\underline{\widehat{\sigma}}_{h}\right) \in \Sigma_{H}$ is defined to be

$$
\Pi_{H}^{\Sigma}\left(\underline{\widehat{\sigma}}_{h}\right)=\Pi_{H}^{\Sigma, E}\left(\underline{\hat{\sigma}}_{h}^{e}\right)+\Pi_{H}^{\Sigma, V}\left(\underline{\widehat{\sigma}}_{h}^{v}\right) .
$$

The following projection error bound follows immediately from (3.19), (3.20), and (3.21).

Proposition 3.3.2 Let $\underline{\widehat{\sigma}}_{h}$ be the snapshot projection of the fine grid stress solution $\underline{\sigma}_{h}$, then

$$
\left\|\underline{\underline{\sigma}}_{h}-\Pi_{H}^{\Sigma}\left(\underline{\hat{\sigma}}_{h}\right)\right\|_{A, \operatorname{div}}^{2} \leq \frac{1}{\Lambda^{E}} \sum_{E_{i}} c_{i}\left(\underline{\hat{\sigma}}_{h}^{e}, \hat{\underline{\sigma}}_{h}^{e}\right)+\frac{1}{\Lambda^{V}} \sum_{V_{i}} d_{i}\left(\underline{\hat{\sigma}}_{h}^{v}, \hat{\underline{\sigma}}_{h}^{v}\right) .
$$

\subsubsection{Inf-sup stability}

Before we move on with the error analysis, we will discuss an inf-sup stability condition for the coarse space pair. Such a condition is indispensable to the convergence of the algorithm. We assume the following conditions hold:

A1. On each coarse edge $E_{i}$, there exits $\underline{\psi}_{1}^{E_{i}}, \underline{\psi}_{2}^{E_{i}} \in \Sigma_{\text {off }}^{E_{i}}$ such that the matrix

$$
\left(\begin{array}{cc}
<\underline{\psi}_{1}^{E_{i}} \cdot \boldsymbol{m}_{i}, \boldsymbol{m}_{i}>_{E_{i}} & <\underline{\psi}_{2}^{E_{i}} \cdot \boldsymbol{m}_{i}, \boldsymbol{m}_{i}>_{E_{i}} \\
<\underline{\psi}_{1}^{E_{i}} \cdot \boldsymbol{m}_{i},\left(x_{2},-x_{1}\right)^{T}>_{E_{i}} & <\underline{\psi}_{2}^{E_{i}} \cdot \boldsymbol{m}_{i},\left(x_{2},-x_{1}\right)^{T}>_{E_{i}}
\end{array}\right)
$$

is invertible.

A2. For each coarse vertex $V_{i}$, let $r_{i}$ be the number of coarse vertices adjacent to $V_{i}$ (depending on location, $r_{i}$ can be 2,3 or 4 ). Let the coarse edges having $V_{i}$ 
as one of the end points be $E_{1}, \ldots, E_{r_{i}}$. Then there exist $\underline{\psi}_{1}^{V_{i}}, \ldots, \underline{\psi}_{r_{i}}^{V_{i}} \in \Sigma_{\text {off }}^{V_{i}}$ such that the matrix

$$
\left(\begin{array}{ccc}
<\underline{\psi}_{1}^{V_{i}} \cdot \boldsymbol{m}_{1}, \boldsymbol{m}_{1}^{\perp}>_{E_{1}} & \cdots & <\underline{\psi}_{r_{i}}^{V_{i}} \cdot \boldsymbol{m}_{1}, \boldsymbol{m}_{1}^{\perp}>_{E_{1}} \\
\vdots & \ddots & \vdots \\
<\underline{\psi}_{1}^{V_{i}} \cdot \boldsymbol{m}_{r_{i}}, \boldsymbol{m}_{r_{i}}^{\perp}>_{E_{r_{i}}} & \cdots & <\underline{\psi}_{r_{i}}^{V_{i}} \cdot \boldsymbol{m}_{r_{i}}, \boldsymbol{m}_{r_{i}}^{\perp}>_{E_{r_{i}}}
\end{array}\right)
$$

is invertible.

With assumptions A1 and A2, we can show the following inf-sup stability condition.

Theorem 3.3.3 There exists a constant $C$ such that for any $\boldsymbol{u}_{H} \in \boldsymbol{U}_{H}$,

$$
\left\|\boldsymbol{u}_{H}\right\| \leq C \sup _{\underline{\tau}_{H} \in \Sigma_{H}} \frac{\left(\operatorname{div}_{h} \underline{\tau}_{H}, \boldsymbol{u}_{H}\right)}{\left\|\underline{\tau}_{H}\right\|_{\operatorname{div}, A}}
$$

Proof. Let $\boldsymbol{u}_{H}$ be an arbitrary element in $\boldsymbol{U}_{H}$. By the inf-sup condition of the fine scale discretization, we know that there is a $\underline{\tau}_{h} \in \Sigma_{h}$ such that

$$
\left\|\boldsymbol{u}_{H}\right\| \leq C_{f} \frac{\left(\operatorname{div}_{h} \underline{\tau}_{h}, \boldsymbol{u}_{H}\right)}{\left\|\underline{\tau}_{h}\right\|_{H(\operatorname{div})}}
$$

where the constant $C_{f}$ is independent of $\boldsymbol{u}_{H}$. Now we will construct an interpolation $\mathscr{J}_{H}: \Sigma_{h} \rightarrow \Sigma_{H}$ such that

$$
\left(\operatorname{div}_{h}\left(\mathscr{J}_{H} \underline{\tau}_{h}-\underline{\tau}_{h}\right), \boldsymbol{u}_{H}\right)=0 \quad \forall \boldsymbol{u}_{H} \in \boldsymbol{U}_{H}
$$

Notice that for each $\boldsymbol{u}_{H} \in \boldsymbol{U}_{H},\left.\boldsymbol{u}_{H}\right|_{K_{j}} \in R M$, so $\boldsymbol{\varepsilon}\left(\left.\boldsymbol{u}_{H}\right|_{K_{j}}\right)=0$. Thus,

$$
\begin{aligned}
\left(\operatorname{div}_{h} \underline{\tau}_{h}, \boldsymbol{u}_{H}\right)_{K_{j}} & =<\underline{\tau}_{h} \cdot \boldsymbol{n}, \boldsymbol{u}_{H}>_{\partial K_{j}}-\left(\underline{\tau}_{h}, \boldsymbol{\varepsilon}\left(\boldsymbol{u}_{H}\right)\right)_{K_{j}} \\
& =<\underline{\tau}_{h} \cdot \boldsymbol{n}, \boldsymbol{u}_{H}>_{\partial K_{j}} .
\end{aligned}
$$

Therefore, condition (3.25) is equivalent to

$$
<\left(\mathscr{J}_{H} \underline{\tau}_{h}-\underline{\tau}_{h}\right) \cdot \boldsymbol{n}, \boldsymbol{u}_{H}>_{\partial E_{i}}=0 \quad \forall E_{i} \in \mathscr{E}_{H}, \forall \boldsymbol{u}_{H} \in \boldsymbol{U}_{H}
$$


To define $\mathscr{J}_{H}$, we first decompose the snapshot projection $\underline{\widehat{\tau}}_{h}=\underline{\widehat{\tau}}_{h}^{e}+\underline{\widehat{\tau}}_{h}^{v}$. Note that since we consider only rectangular grids, $\boldsymbol{u}_{H}$ can be written as

$$
\boldsymbol{u}_{H}=u_{1} \boldsymbol{m}_{i}+u_{2} \boldsymbol{m}_{i}^{\perp}+u_{3}\left(x_{2},-x_{1}\right)^{T} .
$$

locally in a coarse element. For each coarse edge $E_{i},<\underline{\tau}_{h}^{e} \cdot \boldsymbol{m}_{i}, \boldsymbol{m}_{i}^{\perp}>=0$. The same condition holds true also for all basis functions in $\Sigma_{\text {off }}^{E_{i}}$. Moreover, because of the assumption A1, there exist coefficients $\alpha_{1}^{i}, \alpha_{2}^{i}$ such that

$$
\begin{gathered}
<\alpha_{1}^{i} \underline{\psi}_{1}^{E_{i}} \cdot \boldsymbol{m}_{i}+\alpha_{2}^{i} \underline{\psi}_{2}^{E_{i}} \cdot \boldsymbol{m}_{i}, \boldsymbol{m}_{i}>_{E_{i}}=<\underline{\tau}_{h}^{e} \cdot \boldsymbol{m}_{i}, \boldsymbol{m}_{i}>_{E_{i}}, \\
<\alpha_{1}^{i} \underline{\psi}_{1}^{E_{i}} \cdot \boldsymbol{m}_{i}+\alpha_{2}^{i} \underline{\psi}_{2}^{E_{i}} \cdot \boldsymbol{m}_{i},\left(x_{2},-x_{1}\right)^{T}>_{E_{i}}=<\underline{\widehat{\tau}}_{h}^{e} \cdot \boldsymbol{m}_{i},\left(x_{2},-x_{1}\right)^{T}>_{E_{i}} .
\end{gathered}
$$

Hence, by setting

$$
\mathscr{J}_{H}^{e} \underline{\widehat{\tau}}_{h}^{e}=\sum_{E_{i} \in \mathscr{E}_{H}}\left(\alpha_{1}^{i} \underline{\psi}_{1}^{E_{i}}+\alpha_{2}^{i} \underline{\psi}_{2}^{E_{i}}\right)
$$

we get

$$
<\left(\mathscr{J}_{H}^{e} \widehat{\tau}_{h}^{e}-\underline{\tau}_{h}^{e}\right) \cdot \boldsymbol{n}, \boldsymbol{u}_{H}>_{E_{i}}=0 \quad \forall E_{i} \in \mathscr{E}_{H}, \forall \boldsymbol{u}_{H} \in \boldsymbol{U}_{H}
$$

For $\widehat{\tau}_{h}^{e}$, we consider partition of unity $\chi_{i}$ associated with each coarse vertex $V_{i}$. For each coarse vertex $V_{i}$, by the assumption A2, we can find coefficients $\beta_{1}^{i}, \ldots, \beta_{r_{i}}^{i}$ such that

$$
<\left(\beta_{1}^{i} \underline{\psi}_{1}^{V_{i}}+\cdots+\beta_{r_{i}}^{i} \underline{\psi}_{r_{i}}^{V_{i}}\right) \cdot \boldsymbol{m}_{i}, \boldsymbol{m}_{i}^{\perp}>_{E_{j}}=<\left(\underline{\widehat{\tau}}_{h}^{v} \chi_{i}\right) \cdot \boldsymbol{m}_{i}, \boldsymbol{m}_{i}^{\perp}>_{E_{j}} \quad \forall E_{j} \subset \Xi^{V_{i}} .
$$

Note that for any vertex-based basis function $\underline{\psi}_{l}^{V_{i}}$, we have

$$
<\underline{\psi}_{l}^{V_{i}} \cdot \boldsymbol{m}_{i}, u_{1} \boldsymbol{m}_{i}+u_{2} \boldsymbol{m}_{i}^{\perp}+u_{3}\left(x_{2},-x_{1}\right)^{T}>_{E_{i}}=K<\underline{\psi}_{l}^{V_{i}} \cdot \boldsymbol{m}_{i}, \boldsymbol{m}_{i}^{\perp}>_{E_{i}}
$$

where the constant $K=u_{2}+u_{3} x_{2}$ or $K=u_{2}-u_{3} x_{1}$ depending on whether $E_{i}$ is vertical 
or horizontal. The same condition also holds for $\widehat{\tau}_{h}^{v} \chi_{i}$. Thus, it follows that

$$
<\left(\beta_{1}^{i} \underline{\psi}_{1}^{V_{i}}+\cdots+\beta_{r_{i}}^{i} \underline{\psi}_{r_{i}}^{V_{i}}\right) \cdot \boldsymbol{m}_{i}, \boldsymbol{u}_{H}>_{E_{j}}=<\left(\underline{\underline{\tau}}_{h}^{v} \chi_{i}\right) \cdot \boldsymbol{m}_{i}, \boldsymbol{u}_{H}>_{E_{j}} \quad \forall E_{j} \in \mathscr{E}_{H}, \forall \boldsymbol{u}_{H} \in \boldsymbol{U}_{H}
$$

Hence, by setting

$$
\mathscr{J}_{H}^{v} \widehat{\widehat{\tau}}_{h}^{v}=\sum_{V_{i}}\left(\beta_{1}^{i} \underline{\psi}_{1}^{V_{i}}+\cdots+\beta_{r_{i}}^{i} \underline{\psi}_{r_{i}}^{V_{i}}\right)
$$

we get

$$
<\left(\mathscr{J}_{H}^{v} \widehat{\tau}_{h}^{v}-\underline{\tau}_{h}^{v}\right) \cdot \boldsymbol{n}, \boldsymbol{u}_{H}>_{E_{i}}=0 \quad \forall E_{i} \in \mathscr{E}_{H}, \forall \boldsymbol{u}_{H} \in \boldsymbol{U}_{H}
$$

Lastly, we define $\mathscr{J}_{H}=\mathscr{J}_{H}^{e}+\mathscr{J}_{H}^{v}$, then $\mathscr{J}_{H}$ satisfies the desired property (3.25). Now (3.23) follows from (3.24) and (3.25), with the constant $C$ being $C=$ $C_{f}\left\|\mathscr{J}_{H}\right\|$

\subsubsection{Error estimate}

Next, we will estimate the error of the coarse stress solution $\underline{\sigma}_{H}$.

Theorem 3.3.4 Let $\underline{\sigma}_{H}$ and $\underline{\sigma}_{H}$ be the solutions of (3.4) and (3.5), respectively. Then

$$
\left\|\underline{\sigma}_{h}-\underline{\sigma}_{H}\right\|_{A}^{2} \leq C(A)\left(\left\|f-\Pi_{H}^{U} \boldsymbol{f}\right\|^{2}+\frac{1}{\Lambda^{E}} \sum_{E_{i}} c_{i}\left(\underline{\hat{\sigma}}_{h}^{e}, \underline{\hat{\sigma}}_{h}^{e}\right)+\frac{1}{\Lambda^{V}} \sum_{V_{i}} d_{i}\left(\underline{\hat{\sigma}}_{h}^{v}, \underline{\hat{\sigma}}_{h}^{v}\right)\right)
$$

where $\underline{\hat{\sigma}}_{h}^{v}$ and $\underline{\hat{\sigma}}_{h}^{v}$ are projectors defined in Section 3.3.2.

Proof. Since the coarse spaces are subspaces of the respective fine spaces, i.e. $\Sigma_{H} \subset \Sigma_{h}$ and $\boldsymbol{U}_{H} \subset \boldsymbol{U}_{h}$, we can take functions in the coarse spaces as test functions in the fine problem (3.4). Subtracting (3.5) from (3.4), we get

$$
\begin{aligned}
\left(A \underline{\sigma}_{h}-A \underline{\sigma}_{H}, \underline{\tau}_{H}\right) & =\left(\operatorname{div}_{h} \underline{\tau}_{H}, \boldsymbol{u}_{H}-\boldsymbol{u}_{h}\right) & & \forall \underline{\tau}_{H} \in \Sigma_{H}, \\
\left(\operatorname{div}_{h} \underline{\sigma}_{h}-\operatorname{div}_{h} \underline{\sigma}_{H}, \boldsymbol{v}_{H}\right) & =0 & & \forall v_{H} \in \boldsymbol{U}_{H} .
\end{aligned}
$$

Notice that locally in each coarse block $K_{j}, \boldsymbol{v}_{H} \in \boldsymbol{U}_{H}$ is in the space of rigid motion 
$R M$. Hence, from (3.16) and (3.13), we deduce that

$$
\left(\operatorname{div}_{h} \underline{\widehat{\sigma}}_{h}-\operatorname{div}_{h} \underline{\boldsymbol{\sigma}}_{h}, \boldsymbol{v}_{H}\right)=\left(\Pi_{H}^{U} \boldsymbol{f}-\boldsymbol{f}, \boldsymbol{v}_{H}\right)=0 \forall \boldsymbol{v}_{H} \in \boldsymbol{U}_{H}
$$

Moreover, by construction, locally in each coarse block $K_{j}, \operatorname{div}_{h} \underline{\tau}_{H}$ is also in the space of rigid motion $R M$. Thus, by the definition of $\Pi_{H}^{U}$ (3.13), we have

$$
\left(\operatorname{div}_{h} \underline{\tau}_{H}, \boldsymbol{u}_{h}-\Pi_{H}^{U} \boldsymbol{u}_{h}\right)=0 \forall \underline{\tau}_{H} \in \Sigma_{H}
$$

Now, (3.28), (3.29) and (3.30) imply

$$
\begin{aligned}
\left(A \underline{\sigma}_{h}-A \underline{\sigma}_{H}, \underline{\tau}_{H}\right) & =\left(\operatorname{div}_{h} \underline{\tau}_{H}, \boldsymbol{u}_{H}-\Pi_{H}^{U} \boldsymbol{u}_{h}\right) & \forall \underline{\tau}_{H} \in \Sigma_{H}, \\
\left(\operatorname{div}_{h} \underline{\widehat{\sigma}}_{h}-\operatorname{div}_{h} \underline{\sigma}_{H}, \boldsymbol{v}_{H}\right) & =0 & \forall \boldsymbol{v}_{H} \in \boldsymbol{U}_{H} .
\end{aligned}
$$

By taking $\underline{\tau}_{H}=\Pi_{H}^{\Sigma} \underline{\widehat{\sigma}}_{h}-\underline{\sigma}_{H}$ and $\boldsymbol{v}_{H}=\boldsymbol{u}_{H}-\Pi_{H}^{U} \boldsymbol{u}_{h}$ in (3.31), we get

$$
\left(A \underline{\sigma}_{h}-A \underline{\sigma}_{H}, \Pi_{H}^{\Sigma} \underline{\hat{\sigma}}_{h}-\underline{\sigma}_{H}\right)=\left(\operatorname{div}_{h} \Pi_{H}^{\Sigma} \underline{\hat{\sigma}}_{h}-\operatorname{div}_{h} \underline{\widehat{\sigma}}_{h}, \boldsymbol{u}_{H}-\Pi_{H}^{U} \boldsymbol{u}_{h}\right) .
$$

Thus,

$$
\left\|\underline{\sigma}_{h}-\underline{\sigma}_{H}\right\|_{A}^{2} \leq\left\|\underline{\sigma}_{h}-\Pi_{H}^{\Sigma} \underline{\widehat{\sigma}}_{h}\right\|_{A}\left\|\underline{\sigma}_{h}-\underline{\sigma}_{H}\right\|_{A}+\left\|\operatorname{div}_{h}\left(\Pi_{H}^{\Sigma} \underline{\hat{\sigma}}_{h}-\underline{\widehat{\sigma}}_{h}\right)\right\|\left\|u_{H}-\Pi_{H}^{U} \boldsymbol{u}_{h}\right\| .
$$

By the inf-sup condition (3.23) and the first equation of (3.31), we get

$$
\left\|\boldsymbol{u}_{H}-\Pi_{H}^{U} \boldsymbol{u}_{h}\right\| \leq C\left\|\underline{\sigma}_{h}-\underline{\sigma}_{H}\right\|_{A}
$$

Finally, (3.27) follows from (3.15), (3.22), (3.32) and (3.33).

\subsection{Numerical results}

We will perform a series of experiments, and the setting of the experiment is as follows. We take $\Omega=(0,1)^{2}$, and the first Lamé constant $\lambda(x)$ as a piecewise-constant function with heterogeneity pattern as shown in Figure 2.6(a). In all the experiment, 
the value of $\mu$ is always 1 in $\Omega$. The value of $\lambda(x)$ will be different in each set of experiments, but the pattern of heterogeneity will be kept the same. The parameters $L^{E_{i}}$ and $L^{V_{j}}$ are uniform for all coarse edges $E_{i}$ and coarse vertex $V_{j}$, so we will drop the subscript $i$ and $j$. If we use $L^{E}$ multiscale basis functions on each coarse edge, then we use $L^{V}=L^{E}+1$ multiscale basis functions in each coarse neighborhood. We increase $L^{E}$ (and $L^{V}$ will increase accordingly) and see the convergence of the method.

\subsubsection{Snapshot error}

In the first set of experiments, we want to illustrate the snapshot error $\| \underline{\sigma}_{h}-$ $\widehat{\widehat{\sigma}}_{h} \|_{A}(3.15)$, which is irreducible even when $L^{E_{i}}$ and $L^{V_{i}}$ are large. We take $\boldsymbol{f}=\boldsymbol{f}_{1}=$ $(\sin (10 x), \cos (10 y))^{T}$, so $\left\|\boldsymbol{f}_{1}-\Pi_{H}^{U} \boldsymbol{f}_{1}\right\| \neq 0$. Moreover, we take the coarse and fine grids to be a uniform $16 \times 16$ and $160 \times 160$ rectangular grid respectively. So the local fine grid in one coarse element is $10 \times 10$. The first Lamé constant is taken to be $\lambda(x)=10^{3}$ in the red region, and $\lambda(x)=1$ in the blue region (see Figure 2.6). We increase the dimension of the coarse space $\Sigma_{H}$ by increasing $L^{E_{i}}$ and $L^{V_{i}}$, then we plot the relative weighted $L^{2}$ errors of the coarse stress solution $\underline{\sigma}_{H}$ with respect to the fine solution $\underline{\sigma}_{h}$ in Figure 3.2. In the same plot, we draw a red horizontal line to indicate the relative weighted $L^{2}$ errors of the snapshot solution $\widehat{\widehat{\sigma}}_{h}$ with respect to the fine solution $\underline{\sigma}_{h}$. We can see that at first the relative error of the coarse solution decreases rapidly as the dimension of the coarse space is increased, then the error stagnates around the snapshot error 0.035566 . Figure 3.2 clearly shows the existence of the snapshot error and the fact that it cannot be reduced by increasing $L^{E_{i}}$ and 


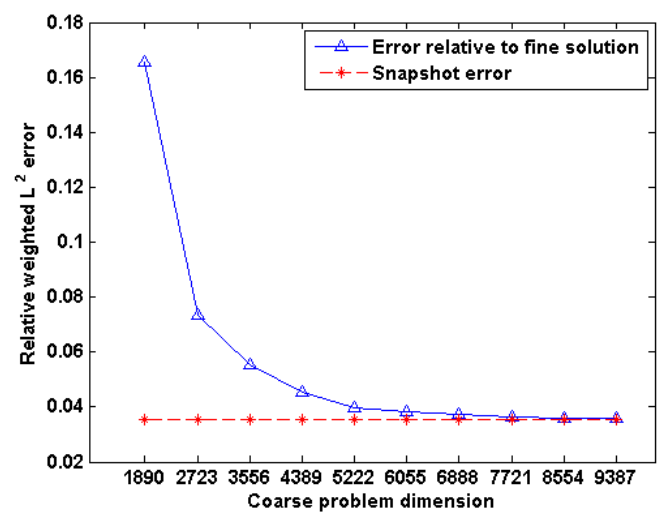

Figure 3.2: Error plot of the mixed GMsFEM approximation of the stress tensor relative to fine scale solution

$L^{V_{i}}$

\subsubsection{Convergence against local enrichment}

In the second set of experiments, we want to examine the convergence of the mixed GMsFEM with respect to the local enrichment (i.e., increment of $L^{E_{i}}$ and $L^{V_{i}}$ ). From the first set of experiment, we know that snapshot error exists and cannot be reduced by increasing $L^{E_{i}}$ and $L^{V_{i}}$. In order to demonstrate other factors which affect the coarse solution error $\left\|\underline{\sigma}_{h}-\underline{\sigma}_{H}\right\|_{A}$, we will set $\boldsymbol{f}=\boldsymbol{f}_{2}=(1,-1)$ from now on. Note that $\left\|\boldsymbol{f}_{2}-\Pi_{H}^{U} \boldsymbol{f}_{2}\right\|=0$, so the snapshot error $\left\|\underline{\sigma}_{h}-\underline{\widehat{\sigma}}_{h}\right\|_{A}$ is also zero in this case. Indeed, the snapshot error is of order $10^{-8}$ in our numerical experiments. Hence, from our analysis, the weighted $L^{2}$ error of the coarse solution depends solely on local enrichment. We take the coarse and fine grids to be a uniform $10 \times 10$ and $100 \times 100$ rectangular grid, respectively. Thus, the local fine grid in one coarse element is again $10 \times 10$. The dimension of the fine problem is 50401 . We take 
$\lambda(x)$ to be the same as in Section 3.4.1. The weighted $L^{2}$ error of the stress tensor and the $L^{2}$ error of the displacement are reported in Table 3.1. We observe rapid

Table 3.1: Experiment set 2.

\begin{tabular}{|c||c|c|}
\hline $\operatorname{dim}\left(\Sigma_{H} \times U_{H}\right)$ & Rel. $L_{2}$ err. for $\sigma$ & Rel. $L_{2}$ err. for $u$ \\
\hline 762 & 0.146198 & 0.114323 \\
1103 & 0.076343 & 0.112576 \\
1444 & 0.049298 & 0.112389 \\
1785 & 0.039348 & 0.112366 \\
2126 & 0.029098 & 0.112356 \\
2467 & 0.016426 & 0.112352 \\
2808 & 0.013899 & 0.112351 \\
3149 & 0.011773 & 0.112351 \\
3490 & 0.009851 & 0.112351 \\
3831 & 0.008899 & 0.112351 \\
\hline
\end{tabular}

convergence in the relative weighted $L^{2}$ error of $\underline{\sigma}_{H}$. In particular, when the coarse problem dimension is 1103 (approximately only 1/50 of the fine problem dimension), the relative error is already 7.6\%. Figure 3.3 shows a comparison plot of the fine and coarse stress solutions. On the other hand, the relative $L^{2}$ error of $\boldsymbol{u}_{H}$ almost does not decrease at all, this is expected as we did not enrich the coarse space $\boldsymbol{U}_{H}$.

\subsubsection{Nearly incompressible material}

In the third set of experiments, we want to investigate the performance of the mixed GMsFEM when the medium a mixture of material with a moderate Poisson's ratio and nearly incompressible material. We take $\lambda(x)=10^{9}$ in the red region, and $\kappa(x)=1$ in the blue region. In this case, the red region represents a nearly incompressible material (Poisson's ratio $v$ close to 0.5 ). The weighted $L^{2}$ errors of 

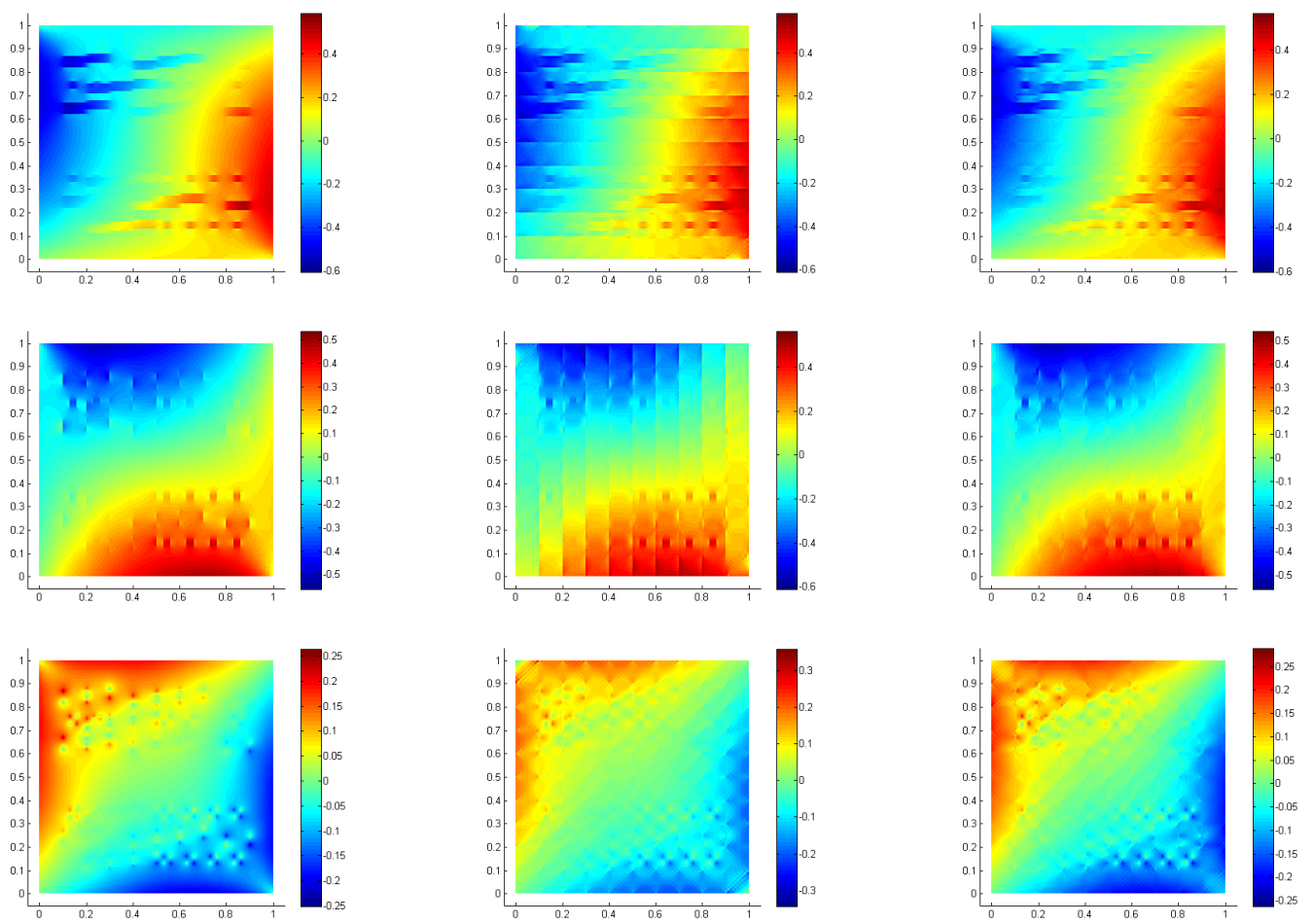

Figure 3.3: Top: $\sigma_{11}$, middle: $\sigma_{22}$, bottom: $\sigma_{12}$, left: fine solution $(\operatorname{dim}=50401$, middle: coarse solution $(\operatorname{dim}=762)$, right: coarse solution $(\operatorname{dim}=1103)$.

the stress tensor and the $L^{2}$ error of the displacement are recorded in Table 3.2. We see that the mixed GMsFEM is robust even when the medium has a very high contrast in the Poisson's ratio. In the last set of experiments, $\lambda(x)=10^{9}$ in the red region, and $\lambda(x)=10^{6}$ in the blue region. So both red and blue regions are nearly incompressible materials, but the Poisson's ratio in red region is closer to 0.5. The weighted $L^{2}$ errors of the stress tensor and the $L^{2}$ error of the displacement are recorded in Table 3.3. Again, we observe robust performance of the mixed GMsFEM in this case. 
Table 3.2: Experiment set 3, high Poisson's ratio only in red region.

\begin{tabular}{|c||c|c|}
\hline $\operatorname{dim}\left(\Sigma_{H} \times U_{H}\right)$ & Rel. $L_{2}$ err. for $\sigma$ & Rel. $L_{2}$ err. for $u$ \\
\hline 762 & 0.146322 & 0.114340 \\
1103 & 0.076518 & 0.112587 \\
1444 & 0.049423 & 0.112398 \\
1785 & 0.039442 & 0.112376 \\
2126 & 0.029167 & 0.112365 \\
2467 & 0.016472 & 0.112361 \\
2808 & 0.013938 & 0.112361 \\
3149 & 0.011806 & 0.112361 \\
3490 & 0.009878 & 0.112361 \\
3831 & 0.008924 & 0.112361 \\
\hline
\end{tabular}

Table 3.3: Experiment set 4, high Poisson's ratio in both regions.

\begin{tabular}{|c||c|c|}
\hline $\operatorname{dim}\left(\Sigma_{H} \times U_{H}\right)$ & Rel. $L_{2}$ err. for $\sigma$ & Rel. $L_{2}$ err. for $u$ \\
\hline 1103 & 0.009956 & 0.085874 \\
1785 & 0.003292 & 0.085712 \\
2467 & 0.001977 & 0.085699 \\
3149 & 0.000923 & 0.085698 \\
3831 & 0.000185 & 0.085698 \\
\hline
\end{tabular}




\section{MULTILEVEL COARSE SPACE CONSTRUCTION BY $\rho$ AMGE}

This section builds upon previous results on numerical upscaling coming from the multiscale finite element approach (cf., [37]) and element based algebraic multigrid approach (AMGe) (cf. [67]). The major difference between the method in this section and the mixed GMsFEM is that, we are going to enrich both the pressure space and velocity space at the same time. Moreover, we will see that the $\rho \mathrm{AMGe}$ framework allows the algorithm to be applied recursively, which results in a multilevel method. The algorithm that we are going to discuss in this section is one of the methods described in [52]. The other method in [52] has a more local construction and therefore more suitable for parallelism. In fact, a slight modification of mixed GMsFEM and the method that we are going to describe in detail can also lead to a local construction for both coarse pressure and velocity spaces. But since parallel implementation is not the main focus of this dissertation, we are not going to discuss too much in that direction.

We build upon developments in the areas exploiting the spectral choice of the degrees of freedom (cf. e.g., [10, 17, 18, 56, 40, 41, 36, 11]), originally proposed for symmetric positive definite (s.p.d.) problems coming from $H^{1}$-conforming finite element discretizations of second order elliptic equations. More recently, building coarse spaces via spectral problems has also been an active research topic in the domain decomposition (DD) community (cf. e.g., [34, 65, 64, 54]), see also, [71, 72]. 
While in the DD area, the main goal is to design DD solvers that are robust with respect to coefficient variations for broad classes of PDEs, our goal is to construct coarse spaces with guaranteed approximation properties so that they can be used as discretization (upscaling) tool. This is motivated and explained in more details in the survey [68] which deals with the use of appropriate AMG-based coarse spaces as accurate discretization spaces (i.e., as an upscaling tool).

The proposed method is another generalization of mixed multiscale finite element method [20,2] because our coarse spaces already contain the coarse spaces in those methods. More specifically, if one pressure basis is picked per agglomerate and one velocity basis is picked per coarse face, the same coarse spaces of the mixed multiscale finite element method are obtained. This additional flexibility in the selection of the number of coarse degrees of freedom allows to fine tune the trade-off between accuracy and computational cost. In addition, as explained in Section 2, high-cost setup can be justified if these spaces are used multiple times. This is the case for our target applications (reservoir simulation, uncertainty quantification) that require solving coarse discretization problems of the type considered in this section many times, while the setup is performed only once and can be viewed as off-line cost.

The specific objective of the present section is to extend the spectral method, originally designed for s.p.d. problems, to mixed finite element discretizations of second order elliptic equations, which is an important advancement of the exist- 
ing numerical upscaling techniques since it is mass-conservative, a desired feature in practical applications such as in porous media flow simulations. The spectral method allows us to discretize and solve the problem at different scales of spatial resolution: by imposing a stronger tolerance when solving the local eigenproblems, we select additional coarse degrees of freedom (dofs) thus improving the approximation properties of the resulting coarse spaces. This feature is an essential component of the methodology developed in [58] (see also earlier results in [62], [57]) to coarsen the entire de Rham fine-grid finite element complex of $L^{2}$-conforming, $H$ (div)-conforming, $H$ (curl)-conforming, and $H^{1}$-conforming spaces with approximation properties. More specifically, the spectral method allows improving the approximation properties of the resulting coarse de Rham complex and to have coarse spaces at different scales of resolution.

In this section, we focus on the part of the complex that involves the $H$ (div) and $L^{2}$-conforming spaces needed for upscaling the mixed finite element discretization of second order elliptic problems of our interest. We note that in some earlier works $[24,16]$, multiscale velocity spaces are constructed and piecewise constant pressure basis functions are used in a two-level setting. 


\subsection{The mixed finite element problem}

We again consider Darcy's flow described by a first-order system of differential equations posed on a given polygonal (or polyhedral) domain $\Omega \subset \mathbb{R}^{d}(d=2$ or 3 ),

$$
\begin{aligned}
\kappa^{-1} \boldsymbol{v}+\nabla p & =\mathbf{0} & & \text { in } \Omega, \\
\operatorname{div} \boldsymbol{v} & =f & & \text { in } \Omega, \\
p & =g_{D} & & \text { on } \Gamma_{D}, \\
\boldsymbol{v} \cdot \boldsymbol{n} & =g_{N} & & \text { on } \Gamma_{N} .
\end{aligned}
$$

The notations are same as in Section 2. However, more generic boundary conditions are considered here. The boundary of $\Omega$ is split into two given complementary pieces, $\Gamma_{D}$ and $\Gamma_{N}$ and we denote with $\boldsymbol{n}$ the unit normal vector to $\Gamma_{N}$ pointing outside $\Omega$. If $\Gamma_{D}$ is empty, as it is well-known, the pressure $p$ is determined up to a (additive) constant (provided $\int_{\Omega} f d \boldsymbol{x}=\int_{\partial \Omega=\Gamma_{N}} g_{N} \mathrm{~d} \boldsymbol{\sigma}$ ). For simplicity, we will use the same notations for discrete and continuous variables, if it will cause no confusion.

We define the following bilinear forms, for a given domain $D$ and boundary $\Gamma$,

$$
\begin{array}{ll}
(p, q)_{D}=\int_{D} p q \mathrm{~d} \boldsymbol{x}, & <\mu, \eta>_{\Gamma}=\int_{\Gamma} \mu \eta \mathrm{d} \boldsymbol{\sigma} \\
a_{D}(\boldsymbol{v}, \boldsymbol{w})=\left(\boldsymbol{\kappa}^{-1} \boldsymbol{v}, \boldsymbol{w}\right)_{D}, & b_{D}(\boldsymbol{v}, q)=-(\operatorname{div} \boldsymbol{v}, q)_{D}
\end{array}
$$

We will drop the subscripts in $(., .)_{D}, a_{D}$ and $b_{D}$ when $D=\Omega$. Similarly, the subscript in $\left\langle., .>_{\Gamma}\right.$ will be dropped when $\Gamma=\Gamma_{D}$. The weak formulation of $(4.1)$ is to look for $(\boldsymbol{v}, p) \in H(\operatorname{div} ; \Omega) \times L^{2}(\Omega)$ such that $\boldsymbol{v} \cdot \boldsymbol{n}=g_{N}$ on $\Gamma_{N}$, and

$$
\begin{array}{rlrl}
a(\boldsymbol{v}, \boldsymbol{w})+b(\boldsymbol{w}, p) & =\left\langle-g_{D}, \boldsymbol{w} \cdot \boldsymbol{n}\right\rangle & & \forall \boldsymbol{w} \in H_{D}(\operatorname{div} ; \Omega), \\
b(\boldsymbol{v}, v) & =(-f, v) & \forall v \in L^{2}(\Omega) .
\end{array}
$$

Here, $H_{D}(\operatorname{div} ; \Omega)=\left\{\boldsymbol{v} \in H(\operatorname{div} ; \Omega) \mid \boldsymbol{v} \cdot \boldsymbol{n}=0\right.$ on $\left.\Gamma_{D}\right\}$. (4.2) is discretized by the 
Raviart-Thomas elements for the velocity, $\boldsymbol{v}$, and piecewise discontinuous polynomials for the pressure, $p$. Let the fine scale approximation space for velocity and pressure be $\boldsymbol{V}_{h}=\operatorname{span}\left\{\boldsymbol{w}_{h, i}\right\}$ and $Q_{h}=\operatorname{span}\left\{q_{h, j}\right\}$ respectively. As a result, we get the global matrices $W, M$, and $B^{T}$, which correspond to the respective bilinear forms $\left(q_{h, i}, q_{h, j}\right), a\left(\boldsymbol{w}_{h, i}, \boldsymbol{w}_{h, j}\right)$, and $b\left(\boldsymbol{w}_{h, i}, q_{h, j}\right)$. The resulting discrete system takes the following saddle-point form

$$
\left[\begin{array}{cc}
M & B^{T} \\
B & 0
\end{array}\right]\left[\begin{array}{l}
\boldsymbol{v} \\
\boldsymbol{p}
\end{array}\right]=\left[\begin{array}{l}
-\boldsymbol{g}_{D} \\
-\boldsymbol{f}
\end{array}\right],
$$

where $\boldsymbol{g}_{D}$ and $\boldsymbol{f}$ come from the Dirichlet boundary conditions and the source term, respectively. We will also need (later on) the traces of the velocity space $\boldsymbol{V}_{h}$ on any interface $F$ (union of fine-grid element faces $f$ ). We denote this space as $\boldsymbol{V}_{h}(F)$. Since, we consider $\boldsymbol{V}_{h}(F)$ in the discrete case only, we view it as a subspace of $L^{2}(F)$. A natural computational basis of the trace space $\boldsymbol{V}_{h}(F)$ is spanned by the fine-grid basis functions (restricted to $F$ ), $\left\{\left.\boldsymbol{w}_{h, i} \cdot \boldsymbol{n}\right|_{F}\right\}$, with degrees of freedom associated with each fine-grid face $f$ that forms $F$.

To formulate a corresponding coarse matrix problem, we construct a prolongation matrix. In terms of coarse-to-fine mapping, the prolongation matrix admits the block diagonal form

$$
P=\left[\begin{array}{cc}
P_{\boldsymbol{v}} & 0 \\
0 & P_{p}
\end{array}\right] .
$$

Here, $P_{v}: V_{H} \rightarrow \boldsymbol{V}_{h}$ and $P_{p}: Q_{H} \rightarrow Q_{h}$ are the prolongation matrices for the velocity space and the pressure space, respectively. Here and in what follows, the subscripts $h$ 
and $H$ denote entities definited on the fine and coarse grid, respectively. The detailed construction of the two matrices will be discussed in Section 4.2.

Once $P$ is constructed, the coarse upscaled problem is obtained variationally from the fine-grid one using the standard "RAP" procedure, namely, if $\mathscr{A}_{h}$ is the finegrid saddle-point matrix in (4.3), the coarse saddle-point matrix $\mathscr{A}_{H}$ equals $P^{T} \mathscr{A}_{h} P$. In practice, the construction of the coarse operator is performed locally on each agglomerate using "RAP" with the local basis of the coarse spaces. The global coarse operator is then assembled from the local coarse matrices using the mapping from agglomerated elements to coarse degrees of freedom. The special AMGe, agglomerated element-by-agglomerated element, construction of the coarse basis and coarse element matrices allows to generate a multilevel hierarchy of nested coarse spaces by applying recursion to further upscale the current coarse level problem. The ability to apply recursion is one of the main features that distinguishes the element agglomeration algebraic multigrid methods (AMGe) - as proposed originally in [51] - from other multiscale finite element techniques.

\subsection{Coarse basis by the spectral AMGe method}

Our goal is to build a coarse-scale discrete model that accurately approximates the fine-scale one. In contrast to the mixed GMsFEM discussed in Section 2, where only accurate coarse velocity space is developed, here we construct coarse spaces with approximation properties for both velocity and pressure. 
Consider a fine mesh $\mathscr{T}_{h}$ and a coarse mesh $\mathscr{T}_{H}$. We will call the elements in $\mathscr{T}_{H}$ coarse elements or agglomerates interchangeably. Different techniques are available to obtain a coarse mesh: graph partitioning techniques, octrees, geometric (coordinate-based) mesh partitioners, and also other techniques that exploit directly the Cartesian or refinement structure of the original fine-grid mesh. To construct agglomerated elements using graph partitioning techniques, in particular, we build the dual graph of the mesh, which is an undirected graph, where each node of the graph represents an element in the mesh and node $i$ is connected to node $j$ if element $i$ and element $j$ share a common face. We use METIS, [53], to obtain a non-overlapping partition of $\mathscr{T}_{h}$ into sets, our agglomerates, which are unions of fine-grid elements. The sets (agglomerates) are assumed connected (if not we split them into connected components), and we define coarse faces $F$, the interfaces between any pair of neighboring agglomerates, in terms of fine-grid faces. By possible further postprocessing, we can ensure that the coarse faces $F$ are also connected sets (as unions of fine-grid faces). Some details of building agglomerates with somewhat regular topology are found in [58]. The set of agglomerates $\mathscr{T}_{H}$ serves as our coarse triangulation (of non-standard elements).

Once the set of agglomerates is available, and the coarse topological relations are constructed (for details cf. [67], Section 1.9), the procedure to construct the coarse spaces can be summarized as follows:

1. Construct a spectral basis for the coarse pressure space $Q_{H}$; 
2. Construct normal traces for the velocity space on coarse faces;

3. Build the coarse velocity space $V_{H}$ from $Q_{H}$ and the velocity traces on coarse faces (from bullet 2).

We emphasize that since our coarse spaces $\left(\boldsymbol{V}_{H}, Q_{H}\right)$ and the topology of coarse elements (i.e., the agglomerates) exhibit the same properties as the fine-grid ones, the above procedure applies recursively, which is a distinct feature of the element agglomeration AMGe approach.

\subsubsection{Constructions of pressure space and velocity normal traces}

We are going to construct the pressure space locally in each coarse element instead of coarse neighborhood. Therefore the construction can naturally be implemented in parallel even in distributed memory architectures.

Before proceeding with the description of the algorithm, let us introduce some notations. To each coarse face $F$, we associate a unit normal vector $\boldsymbol{n}_{F}$. The orientation of $\boldsymbol{n}_{F}$ is arbitrarily chosen and fixed to point outside one of the two agglomerated elements, which share the coarse face $F$. The same convention holds for $\boldsymbol{n}_{f}$, i.e., it is a unit normal vector associated with each fine-grid face $f$ and $\boldsymbol{n}_{f}$ has arbitrary but fixed chosen direction. Let $\varepsilon_{f}=1$ or $\varepsilon_{f}=-1$ depending on whether $\boldsymbol{n}_{f}$ and $\boldsymbol{n}_{F}$ have the same or opposite directions. Then we define the function $\boldsymbol{\phi}_{P V}$, which has constant trace equal to unity when restricted to each coarse face $F$, i.e.,

$$
\boldsymbol{\phi}_{P V}: \boldsymbol{\phi}_{P V} \cdot \boldsymbol{n}_{F}=1 \text { on each } F
$$


It is clear that $\boldsymbol{\mu}_{P V}:=\boldsymbol{\mu}=\left(\boldsymbol{\varepsilon}_{f}\right)_{f \subset F}$ is the coefficient vector in the following expansion

$$
\boldsymbol{\phi}_{P V} \cdot \boldsymbol{n}_{F}=\sum_{f \subset F} \varepsilon_{f} \boldsymbol{\phi}_{f}^{h} \cdot \boldsymbol{n}_{f}
$$

where for each fine-grid face $f, \boldsymbol{\phi}_{f}^{h}$ is the fine-grid basis function that has constant (equal to unity) normal trace. This function was the only coarse velocity degree of freedom (associated with a coarse face $F$ ) used in the paper [62], and it plays an important role in the stability (inf-sup compatibility) of the coarse discretization.

Remark 4.2.1 We remark, that for the development of the method, in particular, for the construction of an important projection operator $\pi_{H}$ that maps fine-grid functions into the coarse space $\boldsymbol{V}_{H}$ (that we construct), the property (4.5) is not necessary (as noticed in [57]); what we need is that the function $\boldsymbol{\phi}_{P V}$ has non-zero face integral, i.e., $\int_{F} \boldsymbol{\phi}_{P V} \cdot \boldsymbol{n}_{F} d \boldsymbol{\sigma} \neq 0$. However, to simplify the exposition, in what follows, we assume that (4.5) holds.

The coarse pressure basis functions are the low frequency eigenvectors of the following spectral problem in each agglomerated element $T$ :

$$
\begin{aligned}
\kappa^{-1} \boldsymbol{v}+\nabla p & =\mathbf{0} & & \text { in } T, \\
\operatorname{div} \boldsymbol{v} & =\lambda p & & \text { in } T, \\
\boldsymbol{v} \cdot \boldsymbol{n} & =0 & & \text { on } \partial T,
\end{aligned}
$$

where $\lambda$ denotes the eigenvalues and $(\boldsymbol{v}, p)$ are the respective eigenfunctions. After elimination of the velocity in the first equation, the matrix representation of the above spectral problem is

$$
B_{T}\left(M_{T}\right)^{-1}\left(B_{T}\right)^{T} \boldsymbol{p}=\lambda W_{T} \boldsymbol{p}
$$


Here $B_{T}$ and $M_{T}$ are the restrictions of the global matrices $B$ and $M$ only to the degrees of freedom associated with the interior of $T$, since $\boldsymbol{v} \cdot \boldsymbol{n}=0$ on $\partial T$. Again, we order the eigenvalues of the spectral problem (4.6) such that $\lambda_{1} \leq \lambda_{2} \leq \ldots \leq \lambda_{n}$. Then given the spectral tolerance $\theta$ and the local maximum number of eigenvectors $k$, the coarse pressure basis functions are taken to be the eigenvectors $\boldsymbol{p}_{i}$ corresponding to the first $m$ eigenvalues, where $m=\max \left\{i: 1 \leq i \leq k\right.$ and $\left.\lambda_{i} \leq \theta \lambda_{n}\right\}$.

Notice that in each $T$, the first eigenvector always represents a constant function (corresponding to the eigenvalue $\lambda_{1}=0$ ). Also, all other eigenvectors are average-free since they are $W_{T}$-orthogonal to the first one. We define the local prolongator for the pressure, $P_{T}^{p}=\left[\boldsymbol{p}_{1}, \ldots, \boldsymbol{p}_{m}\right]$. The global pressure prolongation matrix $P_{p}$ is defined to be the block-diagonal matrix,

$$
P_{p}=\left[\begin{array}{cccc}
P_{T_{1}}^{p} & & & \\
& P_{T_{2}}^{p} & & \\
& & \ddots & \\
& & & \\
& & & P_{T_{N}}^{p}
\end{array}\right]
$$

To generate the coarse traces of the $H$ (div)-conforming space on the agglomerated faces, we proceed as follows. Consider two agglomerated elements $T_{+}$and $T_{-}$ sharing one coarse face $F$. We call the union of $T_{+}$and $T_{-}$a coarse neighborhood of $F$, denoted by $\widetilde{T}$. That is, $\widetilde{T}=T_{+} \cup T_{-}$. We also need a matrix, which evaluates the average of a pressure function in $\widetilde{T}$

$$
D_{\widetilde{T}}=1^{T} W_{\widetilde{T}}
$$


where $\mathbf{1}$ is the vector representation of the unity constant function.

The construction of the normal traces for the velocity basis is based on the coarse pressure space. Let $\left\{\boldsymbol{p}_{i}^{+}\right\}_{i=1}^{m_{+}}$be the coarse pressure basis obtained from (4.6) in $T_{+}$. Similarly, let $\left\{\boldsymbol{p}_{i}^{-}\right\}_{i=1}^{m_{-}}$be the coarse pressure basis obtained from (4.6) in $T_{-}$. Then, we form the following matrix

$$
\widetilde{F}_{p}=\left[\begin{array}{cccccccc}
\boldsymbol{p}_{1}^{+} & \boldsymbol{p}_{2}^{+} & \ldots & \boldsymbol{p}_{m_{+}}^{+} & 0 & 0 & \ldots & 0 \\
0 & 0 & \ldots & 0 & \boldsymbol{p}_{1}^{-} & \boldsymbol{p}_{2}^{-} & \ldots & \boldsymbol{p}_{m_{-}}^{-}
\end{array}\right] .
$$

Denote $\left(\widetilde{F}_{p}\right)_{i}$ to be the $i$-th column of $\widetilde{F}_{p}$. For each $\left(\widetilde{F}_{p}\right)_{i}$, we solve the following local mixed problem

$$
\left[\begin{array}{ccc}
M_{\widetilde{T}} & B_{\widetilde{T}}^{T} & 0 \\
B_{\widetilde{T}} & 0 & D_{\widetilde{T}}^{T} \\
0 & D_{\widetilde{T}} & 0
\end{array}\right]\left[\begin{array}{c}
\boldsymbol{v}_{i} \\
\boldsymbol{v}_{i} \\
\boldsymbol{\eta}
\end{array}\right]=\left[\begin{array}{c}
0 \\
W_{\widetilde{T}}\left(\widetilde{F}_{p}\right)_{i} \\
0
\end{array}\right],
$$

Here $B_{\widetilde{T}}, M_{\widetilde{T}}$ and $W_{\widetilde{T}}$ are the restrictions of the matrices $B, M$ and $W$ only to the interior dofs of the coarse neighborhood $\widetilde{T}$. We then collect the degrees of freedom (normal traces) of all the $\boldsymbol{v}_{i}$ 's restricted on the coarse face $F$.

We note that $p_{1}^{+}$and $p_{1}^{-}$represent constant pressures, and they lead to velocity traces on $F$, that define a function $\boldsymbol{\phi}_{P V}$ with the desired property (4.5), or more generally, with the property as commented in Remark 4.2.1.

In general, the collection of all these trace vectors may be linearly dependent. We perform a weighted-SVD to remove the possible linear dependence and to orthonormalize the basis with respect to the $L^{2}(F)$-inner product. We can further reduce the dimension of the obtained trace space by imposing a certain maximal 
number of singular vectors to be selected. The latter helps controlling the operator complexity of the coarse-scale matrix.

\subsubsection{Construction of the velocity space}

Suppose we have constructed the coarse pressure space and the normal traces on coarse faces using one of the approaches in Section 4.2.1. We now proceed to construct the coarse velocity space. The construction consists of two parts. The first part is the extension of the normal traces on coarse faces. Consider a given normal trace $\mu_{j}$ for the velocity space on a coarse face $F . \mu_{j}$ is extended into the (two) neighboring agglomerates $T$, by solving a local Neumann boundary value problem

$$
\begin{aligned}
\kappa^{-1} \boldsymbol{\phi}_{j}^{F}+\nabla \psi & =0 & & \text { in } T, \\
\operatorname{div} \boldsymbol{\phi}_{j}^{F} & =\text { constant } & & \text { in } T, \\
\boldsymbol{\phi}_{j}^{F} \cdot \boldsymbol{n} & =\mu_{j} & & \text { on } F, \\
\boldsymbol{\phi}_{j}^{F} \cdot \boldsymbol{n} & =0 & & \text { on } \partial T \backslash F .
\end{aligned}
$$

The above constant equals $\frac{1}{|T|} \int_{\partial T} \mu_{j} \mathrm{~d} \boldsymbol{\sigma}$.

Secondly, to complete the construction of the coarse basis of $\boldsymbol{V}_{H}$, we generally add bubble basis functions in each agglomerate so that the required compatibility conditions are met. To this end, for each pressure basis function $p_{i}$ orthogonal to constant (i.e., $i \geq 2$ ), we construct a velocity basis function $\boldsymbol{\phi}_{i}^{b}$, which is supported in $T$ (i.e., its normal trace on $\partial T$ is zero), such that $\operatorname{div} \boldsymbol{\phi}_{i}^{b}=p_{i}$, by solving the local 
Neumann boundary value problem

$$
\begin{array}{rlrl}
\kappa^{-1} \boldsymbol{\phi}_{i}^{b}+\nabla \psi & =\mathbf{0} & \text { in } T, \\
\operatorname{div} \boldsymbol{\phi}_{i}^{b} & =p_{i} & & \text { in } T, \\
\boldsymbol{\phi}_{i}^{b} \cdot \boldsymbol{n} & =0 & & \text { on } \partial T .
\end{array}
$$

The above problems are solvable since $p_{i}$, for $i>1$, are orthogonal to constants. Notice that the bubbles $\boldsymbol{\phi}_{i}^{b}$ 's are linearly independent. In fact, assuming that $\sum c_{i} \boldsymbol{\phi}_{i}^{b}=$ 0 , then we have $\sum c_{i} \operatorname{div} \boldsymbol{\phi}_{i}^{b}=\operatorname{div}\left(\sum c_{i} \boldsymbol{\phi}_{i}^{b}\right)=0$, or equivalently $\sum c_{i} p_{i}=0$, which implies that $c_{i}=0$ for all $i$ because $p_{i}$ 's are linearly independent.

This completes the construction of the coarse basis for the velocity space. In summary, we have basis functions $\boldsymbol{\phi}_{j}^{F}$ associated with any given trace $\mu_{j}$ on the coarse faces $F$ (with constant divergence in the neighboring agglomerated elements), which gives rise to a portion $P_{\boldsymbol{v}}^{F}$ of the coarse-to-fine interpolation matrix $P_{\boldsymbol{v}}$. To complete $P_{\boldsymbol{v}}$, we add as additional columns the block-diagonal matrix $P_{\boldsymbol{v}}^{b}$ representing the velocity bubbles $\boldsymbol{\phi}_{i}^{b}$. That is, we have $P_{\boldsymbol{v}}=\left[P_{\boldsymbol{v}}^{F}, P_{\boldsymbol{v}}^{b}\right]$, which together with $P_{p}$ in $(4.7)$, completes the construction of $P$ in (4.4).

\subsubsection{Compatibility of the coarse pair of spaces}

The construction of the basis of the coarse pair of spaces $\boldsymbol{V}_{H}$ and $Q_{H}$ ensures the property that $\operatorname{div} \boldsymbol{V}_{H}=Q_{H}$. Using a similar argument as in [58] (originally in [62], see also [57]), we show next that the following important commutativity result holds.

Theorem 4.2.2 There is a locally constructed projection operator $\pi_{H}: \boldsymbol{V}_{h} \mapsto \boldsymbol{V}_{H}$, 
which, together with the $L^{2}$-projection $Q_{H}: Q_{h} \mapsto Q_{H}$ (which is also local), satisfies the commutativity property:

$$
\operatorname{div} \pi_{H}=Q_{H} \operatorname{div} \text { on } V_{h}
$$

Proof. We will construct the projection $\pi_{H}$ with the desired properties. Let $\boldsymbol{v} \in \boldsymbol{V}_{h}$ be a given fine-grid function. For each coarse face $F$, let $c_{F}$ be the constant such that

$$
\int_{F} \boldsymbol{v} \cdot \boldsymbol{n}_{F} d \boldsymbol{\sigma}=c_{F} \int_{F} \boldsymbol{\phi}_{P V}^{F} \cdot \boldsymbol{n}_{F} d \boldsymbol{\sigma}
$$

where $\boldsymbol{\phi}_{P V}^{F}$ is the coarse basis function defined in (4.5), or more generally the one discussed in Remark $4.2 .1 ; \boldsymbol{\phi}_{P V}^{F}$ is supported in the neighboring agglomerates $T$ and $T^{\prime}$ that share $F$ with constant divergence (cf., [62]). We first form the interpolant

$$
I_{H}^{F} \boldsymbol{v}=\sum_{F} c_{F} \boldsymbol{\phi}_{P V}^{F}
$$

where the coefficients $c_{F}$ are defined in (4.10). We note that $\operatorname{div}\left(I_{H}^{F} \boldsymbol{v}\right)$ is a piecewise constant function due to the definition of $\boldsymbol{\phi}_{P V}^{F}$.

Next, we define another interpolant $I_{H}^{b} \boldsymbol{v}$ such that, on each agglomerate $T$,

$$
\left.\left(I_{H}^{b} \boldsymbol{v}\right)\right|_{T}=\sum_{i=2}^{m} c_{i} \boldsymbol{\phi}_{i}^{b},
$$

where $\boldsymbol{\phi}_{i}^{b}$ are the bubbles on $T$ (see Section 4.2.2) and the constants $c_{i}$ 's are given by

$$
c_{i}=\left(\operatorname{div} \boldsymbol{v}, p_{i}\right)_{T}, \quad \text { for } i=2, \ldots, m
$$

Using (4.10) and the facts that $\operatorname{div}\left(I_{H}^{F} \boldsymbol{v}\right)$ and $p_{1}$ are constant on $T, \operatorname{div} \boldsymbol{\phi}_{i}^{b}=p_{i}$, and $\left\{p_{i}\right\}_{i=1}^{m}$ are orthonormal w.r.t. the $L^{2}$ inner product $(\cdot, \cdot)_{T}$, one can deduce that

$$
\left(\operatorname{div}\left(I_{H}^{F} \boldsymbol{v}+I_{H}^{b} \boldsymbol{v}\right), p_{i}\right)_{T}=\left(\operatorname{div} \boldsymbol{v}, p_{i}\right)_{T}, \quad \text { for } i=1, \ldots, m
$$


To complete the construction of $\pi_{H}$, we will need the last interpolant $I_{H}^{0} \boldsymbol{v}$ such that, on each coarse face $F,\left.\left(I_{H}^{0} \boldsymbol{v}\right)\right|_{F}=\sum_{i=1}^{n_{F}} c_{i}^{0} \boldsymbol{\phi}_{H, i}^{F}$, and for all $i=1, \ldots, n_{F}$,

$$
<\left(I_{H}^{0} \boldsymbol{v}\right) \cdot \boldsymbol{n}_{F}, \boldsymbol{\phi}_{H, i}^{F} \cdot \boldsymbol{n}_{F}>_{F}=<\boldsymbol{v} \cdot \boldsymbol{n}_{F}, \boldsymbol{\phi}_{H, i}^{F} \cdot \boldsymbol{n}_{F}>_{F},
$$

where $\boldsymbol{\phi}_{H, i}^{F}$ are the remaining $F$-based coarse basis functions (each comes from a coarse normal trace on $F$ ) other than $\boldsymbol{\phi}_{P V}^{F}$. Without loss of generality, we may assume that $\int_{F} \boldsymbol{\phi}_{H, i}^{F} \cdot \boldsymbol{n}_{F} d \boldsymbol{\sigma}=0$. To ensure this, we can replace $\boldsymbol{\phi}_{H, i}^{F}$ by $\boldsymbol{\phi}_{H, i}^{F}-c \boldsymbol{\phi}_{P V}^{F}$ with an appropriate value of $c$ if necessary. This implies that $\operatorname{div}\left(\boldsymbol{\phi}_{H, i}^{F}\right)=0$ (recall that $\operatorname{div}\left(\boldsymbol{\phi}_{H, i}^{F}\right)$ is piecewise constant). Since $\boldsymbol{\phi}_{H, i}^{F} \cdot \boldsymbol{n}_{F}$ are coming from SVD on $L^{2}(\boldsymbol{F})$, they are linearly independent, hence (4.13) is uniquely solvable.

Finally, we set $\pi_{H} \boldsymbol{v}=I_{H}^{F} \boldsymbol{v}+I_{H}^{b} \boldsymbol{v}+I_{H}^{0} \boldsymbol{v}$. Then $\pi_{H}$ is a projection on $\boldsymbol{V}_{H}$, i.e., $\pi_{H} \boldsymbol{v}_{H}=\boldsymbol{v}_{H}, \forall \boldsymbol{v}_{H} \in \boldsymbol{V}_{H}$. Also, since $\operatorname{div}\left(I_{H}^{0} \boldsymbol{v}\right)=0$, (4.12) implies that

$$
\left(\operatorname{div}\left(\pi_{H} \boldsymbol{v}\right), p_{H}\right)=\left(\operatorname{div} \boldsymbol{v}, p_{H}\right), \quad \forall p_{H} \in Q_{H}
$$

Hence, the commutativity property (4.9) holds.

\subsection{Approximation properties of the coarse pressure spaces}

We now analyze the approximation properties of the coarse space $Q_{H}$. To this aim let us introduce the discrete $H^{1}$-like norm for finite element functions $p_{h} \in Q_{h}$ defined as $\left\|p_{h}\right\|_{*}^{2}=\sum_{T \in T_{H}} \boldsymbol{p}^{T} B_{T}\left(M_{T}\right)^{-1}\left(B_{T}\right)^{T} \boldsymbol{p}$, where $\boldsymbol{p}$ is the coefficient vector of $p_{h}$ expanded in terms of the basis functions in $Q_{h}$. The following result establishes the approximation properties of the coarse space $Q_{H}$ with respect to the local spectral tolerance $\theta$ and the local maximum number of eigenvectors $k$. 
Theorem 4.3.1 Let the coarse space $Q_{H}$ be constructed with the spectral tolerance $\theta$ and the local maximum number of eigenvectors $k$, then

$$
\inf _{p_{H} \in Q_{H}}\left\|p_{h}-p_{H}\right\|_{L^{2}(\Omega)}^{2} \leq \max _{T \in \mathscr{T}_{H}}\left(\frac{1}{\lambda_{m+1}(T)}\right)\left\|p_{h}\right\|_{*}^{2} \quad \forall p_{h} \in Q_{h} .
$$

Here $\lambda_{m+1}(T)$ is the smallest eigenvalue of (4.6) whose eigenfunction is not included in the local coarse pressure space. More precisely, $m=\max \left\{i: 1 \leq i \leq k\right.$ and $\left.\lambda_{i} \leq \theta \lambda_{n}\right\}$ is the dimension of the local coarse pressure space defined on the agglomerated element $T \in \mathscr{T}_{H}$

Proof. Since the coarse pressure space is a direct sum of local eigenspaces in each agglomerate, it suffices to look at one agglomerate $T$.

Consider any function $p_{h}$ in the fine scale pressure space $Q_{h}$ restricted to $T$. Let $\boldsymbol{p}$ be the coefficient vector of $p_{h}$ expanded in terms of the basis functions in $Q_{h}$. The eigenvectors $\boldsymbol{p}_{i}, 1 \leq i \leq n$ form an orthonormal basis for the local fine scale space. Hence, there exists some $c_{i}$ 's such that

$$
\boldsymbol{p}=\sum_{i=1}^{n} c_{i} \boldsymbol{p}_{i}
$$

We define an interpolant of $\boldsymbol{p}, I_{H}(\boldsymbol{p})$, as $I_{H}(\boldsymbol{p})=\sum_{i=1}^{m} c_{i} \boldsymbol{p}_{i}$, where $m=\max \{i: 1 \leq i \leq$ $k$ and $\left.\lambda_{i} \leq \theta \lambda_{n}\right\}$ is the number of eigenvectors that we have included in the coarse pressure space, so $\left.I_{H}(\boldsymbol{p}) \in Q_{H}\right|_{T}$. Using the orthogonality of eigenvectors and the 
ordering of eigenvalues, we have

$$
\begin{aligned}
\left(\boldsymbol{p}-I_{H}(\boldsymbol{p})\right)^{T} W_{T}\left(\boldsymbol{p}-I_{H}(\boldsymbol{p})\right) & \leq\left\|\boldsymbol{p}-I_{H}(\boldsymbol{p})\right\|_{W_{T}}^{2} \\
& =\left\|\boldsymbol{p}-\sum_{i=1}^{m} c_{i} \boldsymbol{p}_{i}\right\|_{W_{T}}^{2} \\
& =\sum_{i=m+1}^{n} c_{i}^{2} \boldsymbol{p}_{i}^{T} W_{T} \boldsymbol{p}_{i} \\
& =\sum_{i=m+1}^{n} \frac{1}{\lambda_{i}} c_{i}^{2} \boldsymbol{p}_{i}^{T} B_{T} M_{T}^{-1} B_{T}^{T} \boldsymbol{p}_{i} \\
& \leq \frac{1}{\lambda_{m+1}} \boldsymbol{p}^{T} B_{T} M_{T}^{-1} B_{T}^{T} \boldsymbol{p}
\end{aligned}
$$

It follows that

$$
\inf _{p_{H} \in Q_{H}}\left\|p_{h}-p_{H}\right\|_{L^{2}(T)}^{2} \leq\left\|\boldsymbol{p}-I_{H}(\boldsymbol{p})\right\|_{W_{T}}^{2} \leq \frac{1}{\lambda_{m+1}(T)} \boldsymbol{p}^{T} B_{T} M_{T}^{-1} B_{T}^{T} \boldsymbol{p} .
$$

The assertion follows by summing over the agglomerates $T$ in $\mathscr{T}_{H}$.

Remark 4.3.2 In practice, we choose a small number of eigenvectors with the goal to capture portion of the spectrum that is close to zero. We achieve this by using tolerance to split the spectrum into two groups, and we can additionally impose a limit on the size of the set of respective eigenvectors. The latter affects the size and structure of the constructed interpolation matrices, which on the other hand influences the sparsity of the upscaled coarse matrices. For more details, see the numerical experiments section.

\subsection{Stability properties and error analysis}

In this section, we analyze the error of our method in the two-level case. In what follows, we introduce the following notations: for $\boldsymbol{v}_{h} \in \boldsymbol{V}_{h},\left\|\boldsymbol{v}_{h}\right\|_{a}^{2}=a\left(\boldsymbol{v}_{h}, \boldsymbol{v}_{h}\right)$ and $\left\|\boldsymbol{v}_{h}\right\|^{2}=\left\|\boldsymbol{v}_{h}\right\|_{a}^{2}+\left\|\operatorname{div} \boldsymbol{v}_{h}\right\|^{2}$. We assume that the fine-grid problem exhibits the 
following inf-sup condition

$$
\inf _{p_{h} \in Q_{h}} \sup _{\boldsymbol{v}_{h} \in V_{h}} \frac{\left(\operatorname{div} \boldsymbol{v}_{h}, p_{h}\right)}{\left\|\boldsymbol{v}_{h}\right\|\left\|p_{h}\right\|} \geq \beta
$$

Using the projection operator $\pi_{H}$ introduced in Section 4.2.3, we can prove the following inf-sup condition for the coarse pair of spaces $\boldsymbol{V}_{H}, Q_{H}$,

$$
\inf _{p_{H} \in Q_{H}} \sup _{\boldsymbol{v}_{H} \in \boldsymbol{V}_{H}} \frac{\left(\operatorname{div} \boldsymbol{v}_{H}, p_{H}\right)}{\left\|\boldsymbol{v}_{H}\right\|\left\|p_{H}\right\|} \geq \frac{\beta}{\left\|\pi_{H}\right\|},
$$

where $\left\|\pi_{H}\right\|$ is the weighted $H$ (div)-norm of $\pi_{H}$, i.e., $\left\|\pi_{H}\right\|=\sup _{\boldsymbol{v}_{h} \in \boldsymbol{V}_{h}} \frac{\left\|\pi_{H} \boldsymbol{v}_{h}\right\|}{\left\|\boldsymbol{v}_{h}\right\|}$. This estimate is easily seen from the fine-grid inf-sup estimate using the commutativity property (4.9), which gives $\left(\operatorname{div}\left(\pi_{H} \boldsymbol{v}_{h}\right), p_{H}\right)=\left(\operatorname{div} \boldsymbol{v}_{h}, p_{H}\right)$.

Let $\left(\boldsymbol{v}_{h}, p_{h}\right) \in \boldsymbol{V}_{h} \times Q_{h}$ be the solution to the fine (level) problem, and $\left(\boldsymbol{v}_{H}, p_{H}\right) \in$ $\boldsymbol{V}_{H} \times Q_{H}$ be the solution to the coarse (level) problem. Since, we have an inf-sup condition on the coarse level, standard error analysis for the mixed finite element discretization problem applies, which involves the best approximation properties of the pressure space $Q_{H}$ and the velocity $\boldsymbol{V}_{H}$. In particular, the following standard error estimate holds (cf., e.g., Theorem 5.25 in [7], or [63])

$$
\left\|\boldsymbol{v}_{H}-\boldsymbol{v}_{h}\right\|+\left\|p_{H}-p_{h}\right\| \leq C\left(\inf _{\boldsymbol{w}_{H} \in \boldsymbol{V}_{H}}\left\|\boldsymbol{w}_{H}-\boldsymbol{v}_{h}\right\|\left\|+\inf _{v_{H} \in Q_{H}}\right\| v_{H}-p_{h} \|\right),
$$

where the constant $C$, among other things (as shown in [7]), depends on the "inf-sup" bound (which in our case is $\frac{\left\|\pi_{H}\right\|}{\beta}$ ).

With the spectral choice of the coarse degrees of freedom the approximation properties improve with the increase of the spectral tolerance. For the velocity space $\boldsymbol{V}_{H}$, we have the freedom to add as many additional degrees of freedom on each 
coarse face $F$ as we want to. In particular, we can add additionally, traces of vector polynomials (the ones that are contained in the fine-grid space for example), as investigated in [57] and also in [58].

Remark 4.4.1 We note that the norm $\left\|\pi_{H}\right\|$ can actually be estimated numerically by local operations, since the construction of $\pi_{H}$ involves local procedures. One possible way to keep $\left\|\pi_{H}\right\|$ under control, is to proceed in the following adaptive way. We have a current projection mapping $\pi_{H}$ corresponding to one compatible selection of coarse degrees of freedom. On each $T$, we solve the following eigenvalue problem

$$
\lambda_{T}=\max _{\boldsymbol{v} \in \boldsymbol{R}_{h}(T)} \frac{\left\|\pi_{H} \boldsymbol{v}\right\|_{T}^{2}}{\|\boldsymbol{v}\|_{T}^{2}} .
$$

If we encounter on some $T$ a $\lambda_{T}$ that is too large (i.e., larger than a desired upper bound of the norm of $\pi_{H}$ ), we take the traces of the above eigenfunction $\boldsymbol{v}$ on each coarse face $F$, and augment the set of traces on $F$ used to build the coarse basis of $\boldsymbol{V}_{H}$. That leads to a new, modified, projection operator $\pi_{H}$, for which the above eigenvalue problem yields smaller value $\lambda_{T}$. We repeat this adaptive process until the computed $\lambda_{T}$ become smaller than the desired upper bound of $\pi_{H}$. We note, that the eigenvalue problems in Methods 1 and 2 from Section 4.2, have the ability to enrich the set of normal traces, which indirectly leads to better values $\lambda_{T}$ in $(4.15)$ (since we increase the dimension of the trace space included in the coarse space).

Remark 4.4.2 In the case of geometric coarsening, i.e., agglomerates that have regular polyhedral structure, in particular flat faces $F$, under certain assumptions on the 
coefficient $\boldsymbol{\kappa}(\boldsymbol{x})$ (in (4.1)), in [20] it was shown that the coarse inf-sup constant can be bounded independently of the contrast of $\kappa$.

\subsection{Numerical experiments}

We demonstrate the performance of our spectral AMGe upscaling method on a series of experiments. In the following tables, $k$ and $\theta$ are two parameters used to control the number of degrees of freedom of the coarse spaces. $\operatorname{dim}\left(\boldsymbol{V}_{h}\right)$ and $\operatorname{dim}\left(\boldsymbol{V}_{H}\right)$ refer to the number of velocity degrees of freedom in the fine level and coarse level respectively, while $\operatorname{dim}\left(Q_{h}\right)$ and $\operatorname{dim}\left(Q_{H}\right)$ are the counterparts for the pressure

degrees of freedom. OC is the operator complexity computed as $\left(\sum_{k=0}^{\ell-1} \mathrm{nnz}[k]\right) / \mathrm{nnz}[0]$, where $\ell$ is the number of levels, and $n n z[k]$ is the number of non-zeros of the mixed system at level $k$. We use the convention that level 0 represents the original (finest) level. Except in Experiment 3, the lowest order (zero) Raviart-Thomas finite element space is used on the finest level. The coarsening factor refers to the ratio between the numbers of fine grid elements and the coarse grid elements. For structured partitions, the coarsening factor in each coordinate direction is stated.

The numerical results in this section were generated by the $\mathrm{C}++$ libraries MFEM [mfem.org] and ELAG developed in the Lawrence Livermore National Laboratory. 


\subsubsection{D examples}

For all the 2D examples presented in the following, we consider the the top layer of the SPE10 benchmark (for its detailed description, see [21], and also Section 4.5.2). The permeability field is shown in Figure 4.1(a), and the mesh is a structured rectangular grid with elements' sizes of $20 \mathrm{ft} \times 10 \mathrm{ft}$.

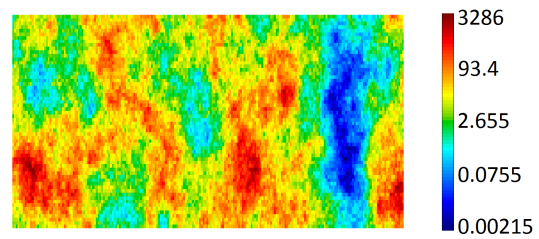

(a) Top layer

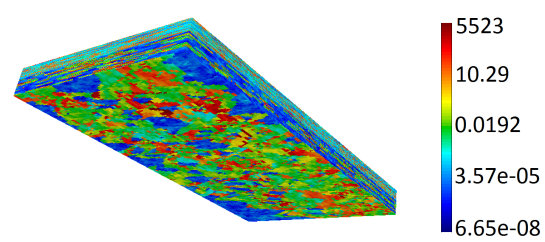

(b) Full model

Figure 4.1: Top layer of the SPE10 model and the full 3D model.

Experiment 1: performance against local enrichment. In the first experiment, we want to demonstrate how the spectral tolerance $\theta$ and the local maximum number of eigenvectors $k$ affect the approximation properties of the upscaled spaces and the complexity of the upscaled model. We fix the fine and coarse mesh to be $60 \times 220$ and $6 \times 22$ structured grids, respectively. The number of degrees of freedom for velocity on the fine level is 26680 and for the pressure is 13200 . In this example we demonstrate the two criteria to locally (agglomerated element by agglomerated element) determine the dimension of the coarse spaces, namely the "pick $\theta$ " and the "choose $k$ " criterion. More specifically, for the first criterion we consider increasing values of the threshold $\theta$ to determine how many eigenmodes are selected in each local problem (see Table 
4.1) and for the second criterion we select the first $k$ eigenmodes from each local spectral problem (see Table 4.2). We remark that in all other experiments to follow, we only present results for the "choose $k$ " criterion which formally corresponds to tolerance $\theta=1$; recall that the actual number of eigenvectors selected by our criterion is $m=\max \left\{i: 1 \leq i \leq k\right.$ and $\left.\lambda_{i} \leq \theta \lambda_{n}\right\}$. In Table 4.1, "** indicates that for the specific choice of $\theta$, there are more than 10 eigenvalues $\lambda_{i}$ satisfying the constraint $\lambda_{i} \leq \theta \lambda_{n}$ in some local spectral problems. However, since we set an upper bound of $k=10$, only 10 eigenvectors are selected in those local spectral problems. Both methods show very good convergence with respect to local enrichment. In particular, the velocity upscaling error is roughly $7.5 \%$ when using the "choose $k$ " criterion with $k=2$. The corresponding operator complexity is 1.05 , which means the memory needed for the storage of the upscaled operator (matrix) is just $5 \%$ of the original one. In Figure 4.2 , we display the velocity solutions obtained from the proposed $\rho$ AMGe upscaling method with different $k$ as well as the reference (fine grid) solution. For ease of comparison, we used the same colorbar for all the pictures. We can clearly see that some features in the reference solution are missing in the coarse solution with $k=$ 1. These features are picked up when $k=5$. We also display the pressure solutions obtained from the proposed method in Figure 4.3. The improved resolution of coarse pressure solution by adding more local basis is evident from the plots.

Experiment 2: performance against coarsening factor. In this experiment, we fix the fine mesh to be $120 \times 440$, and then change the coarsening factor. Notice that 


\begin{tabular}{|c||c|c|c|c|c|}
\hline $\boldsymbol{\theta}$ & $\operatorname{dim}\left(\boldsymbol{V}_{\boldsymbol{H}}\right)$ & $\operatorname{dim}\left(Q_{H}\right)$ & $\mathrm{OC}$ & $\frac{\left\|\boldsymbol{v}_{H}-\boldsymbol{v}_{h}\right\|}{\left\|\boldsymbol{v}_{h}\right\|}$ & $\frac{\left\|p_{H}-p_{h}\right\|}{\left\|p_{h}\right\|}$ \\
\hline $1 \mathrm{e}-4$ & 307 & 135 & 1.01 & 0.1936 & 0.05025 \\
$4 \mathrm{e}-4$ & 676 & 218 & 1.06 & 0.1200 & 0.04921 \\
$7 \mathrm{e}-4^{*}$ & 1064 & 319 & 1.14 & 0.07659 & 0.04878 \\
$1 \mathrm{e}-3^{*}$ & 1371 & 404 & 1.23 & 0.05211 & 0.04227 \\
$1.5 \mathrm{e}-3^{*}$ & 1771 & 508 & 1.36 & 0.02650 & 0.03461 \\
\hline
\end{tabular}

Table 4.1: Experiment 1 with various $\boldsymbol{\theta}$. We have imposed an upper bound on the number of eigenvectors to be used (we let $k=10$ ). For the fine-grid level $\operatorname{dim}\left(\boldsymbol{V}_{h}\right)=$ 26680 and $\operatorname{dim}\left(Q_{h}\right)=13200$.

\begin{tabular}{|c||c|c|c||c|c|}
\hline$k$ & $\operatorname{dim}\left(\boldsymbol{V}_{H}\right)$ & $\operatorname{dim}\left(Q_{H}\right)$ & $\mathrm{OC}$ & $\frac{\left\|\boldsymbol{v}_{H}-\boldsymbol{v}_{h}\right\|}{\left\|\boldsymbol{v}_{h}\right\|}$ & $\frac{\left\|p_{H}-p_{h}\right\|}{\left\|p_{h}\right\|}$ \\
\hline 1 & 292 & 132 & 1.01 & 0.2078 & 0.05035 \\
2 & 716 & 264 & 1.05 & 0.07472 & 0.04858 \\
3 & 1140 & 396 & 1.13 & 0.04843 & 0.02482 \\
4 & 1564 & 528 & 1.23 & 0.02239 & 0.01881 \\
5 & 1988 & 660 & 1.37 & 0.01644 & 0.01254 \\
\hline
\end{tabular}

Table 4.2: Experiment 1 with various $k$. For the fine-grid level, $\operatorname{dim}\left(\boldsymbol{V}_{h}\right)=26680$ and $\operatorname{dim}\left(Q_{h}\right)=13200$.

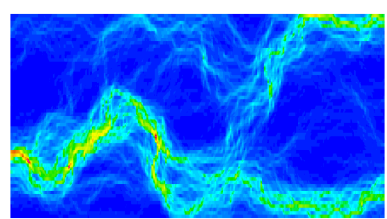

(a) Reference solution

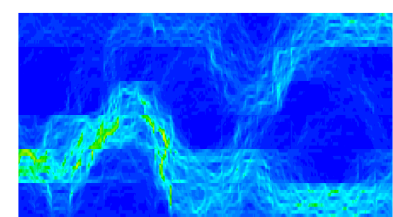

(b) Coarse solution: $k=1$

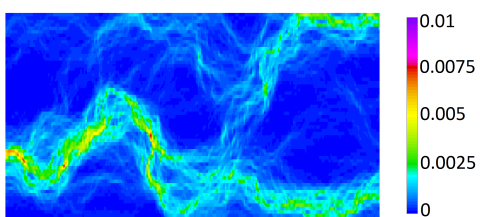

(c) Coarse solution: $k=5$

Figure 4.2: Magnitudes of velocity solutions in Experiment 1.

when we decrease the coarsening factor, the coarse mesh is refined. This means that we actually change the size of the agglomerates and hence the dimensions of the local fine spaces. We adjust the parameter $k$ so that the operator complexity stays more or less the same for different choices of coarsening factors. This is a very interesting and informative experiment as it reveals whether one should opt for $h$-refinement (i.e. 


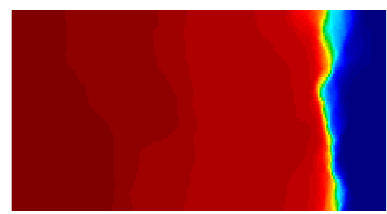

(a) Reference solution

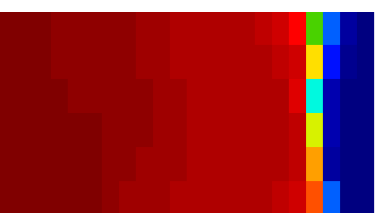

(b) Coarse solution: $k=1$

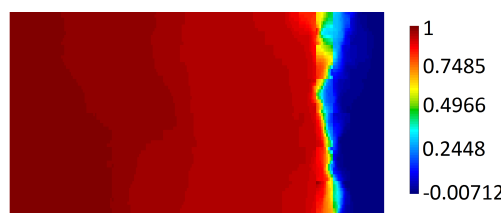

(c) Coarse solution: $k=5$

Figure 4.3: Pressure solutions in Experiment 1.

smaller agglomerates) or p-refinement (i.e., more spectral coarse degrees of freedom). From Table 4.3, we can see that, in general, the choice of a coarser agglomerated mesh and larger local spaces gives a smaller coarse problem (in terms of number of degrees of freedom) with a better approximation. Hence, p-refinement is superior to $h$-refinement in this regard. Nevertheless, a larger (more aggressive) coarsening factor means we need to solve larger local problems to construct the coarse spaces, hence the setup is more expensive.

\begin{tabular}{|c|c||c|c|c||c|c|}
\hline C.F. & $k$ & $\operatorname{dim}\left(V_{H}\right)$ & $\operatorname{dim}\left(Q_{H}\right)$ & OC & $\frac{\left\|v_{H}-\boldsymbol{v}_{h}\right\|}{\left\|\boldsymbol{v}_{h}\right\|}$ & $\frac{\left\|p_{H}-p_{h}\right\|}{\left\|p_{h}\right\|}$ \\
\hline $5 \times 5$ & 2 & 10784 & 4224 & 1.22 & 0.04523 & 0.01326 \\
$10 \times 10$ & 4 & 6032 & 2112 & 1.24 & 0.02555 & 0.01183 \\
$20 \times 20$ & 8 & 3123 & 824 & 1.25 & 0.01092 & 0.006483 \\
\hline
\end{tabular}

Table 4.3: Performance of $\rho$ AMGe upscaling in Experiment 2. C.F. $=$ coarsening factor. For the fine-grid level, $\operatorname{dim}\left(\boldsymbol{V}_{h}\right)=106160$ and $\operatorname{dim}\left(Q_{h}\right)=52800$.

Experiment 3: performance against finite element order. The spectral AMGe upscaling method can be applied to systems arising from higher order finite element discretization of the differential equations. In this experiment, the fine mesh is fixed to be $60 \times 220$, and the coarsening factor is fixed to be $5 \times 5$. When we increase 
the fine level finite element order, we actually increase the local problem size since the coarsening factor stays the same. It is worth to notice that higher order finite element discretizations not only lead to more degrees of freedom, but also to denser matrices. For this reason, for a fixed operator complexity, an increase in the finite element order corresponds to an increase in the number of coarse degrees of freedom. Consequently, we expect a decrease in the upscaling error when the FE order is increased as observed in Table 4.4 .

\begin{tabular}{|c|c||c|c|c||c|c|}
\hline FEO & $k$ & $\operatorname{dim}\left(\boldsymbol{V}_{h} \times Q_{h}\right)$ & $\operatorname{dim}\left(\boldsymbol{V}_{H} \times Q_{H}\right)$ & OC & $\frac{\left\|\boldsymbol{v}_{H}-\boldsymbol{v}_{h}\right\|}{\left\|\boldsymbol{v}_{h}\right\|}$ & $\frac{\left\|p_{H}-p_{h}\right\|}{\left\|p_{h}\right\|}$ \\
\hline 0 & 1 & $26680 \times 13200$ & $1112 \times 528$ & 1.04 & 0.1637 & 0.02799 \\
1 & 2 & $106160 \times 52800$ & $2752 \times 1056$ & 1.02 & 0.07068 & 0.02643 \\
2 & 4 & $238440 \times 118800$ & $6032 \times 2112$ & 1.02 & 0.03050 & 0.01213 \\
\hline
\end{tabular}

Table 4.4: Performance of $\rho \mathrm{AMGe}$ upscaling in Experiment 3. FEO = finite element order.

Experiment 4: multilevel coarsening on a structured hierarchy. As mentioned in Section 4.1, the spectral AMGe upscaling method can be applied recursively and becomes a multilevel method. The advantage of multilevel upscaling is that we can keep the coarsening factor between two consecutive levels small, so the local problems that we solve are kept reasonably small. Consequently, the construction of the coarse spaces is much more efficient. The price to pay is that the errors will generally be larger than the two-level method (when we skip the intermediate levels and jump directly to the final coarse level). The number of elements starting from level 0 to level 3 are 52800, 528, 10 and 1, respectively. Note that the number of degrees of 
freedom at the coarsest level is 29 , while the dimension of the fine problem is 158960 , which means that the reduction in dimension is more than 5000 times. Nevertheless, we can see from Table 4.5 that the relative errors are quite small. We also plot out

\begin{tabular}{|c|c|c|c|c||c|c|}
\hline Level & $N_{K}$ & $\operatorname{dim}\left(\boldsymbol{V}_{H}\right)$ & $\operatorname{dim}\left(P_{H}\right)$ & OC & $\frac{\left\|\boldsymbol{v}_{H}-\boldsymbol{v}_{h}\right\|}{\left\|v_{h}\right\|}$ & $\frac{\left\|p_{H}-p_{h}\right\|}{\left\|p_{h}\right\|}$ \\
\hline 1 & 5 & 7672 & 2640 & 1.38 & 0.01563 & 0.00581 \\
2 & 5 & 175 & 50 & 1.39 & 0.02791 & 0.02933 \\
3 & 5 & 24 & 5 & 1.39 & 0.02791 & 0.03202 \\
\hline
\end{tabular}

Table 4.5: Performance of the $\rho$ AMGe upscaling in Experiment 4. For the fine-grid level, $\operatorname{dim}\left(\boldsymbol{V}_{h}\right)=106160$ and $\operatorname{dim}\left(Q_{h}\right)=52800$.

the solutions in each level in Figure 4.4-4.5. Indeed, the approximations in different coarse levels look very close to the fine level reference solutions.

Experiment 5: multilevel coarsening on an unstructured hierarchy. In this experiment, we demonstrate the ability of the spectral AMGe upscaling method to handle unstructured grids, which is common in many practical applications. In such situations, the coarse grid will definitely be unstructured as well. An unstructured hierarchy of meshes is then built such that the coarser level corresponds to an unstructured partition of the previous finer one; see Figure 4.6 for an illustration. Such agglomerated elements are generated using graph partitioning algorithms (METIS) leading to agglomerated elements with arbitrary shapes, non-planar faces, and number of neighboring elements. These irregularities in the coarse topology usually lead to an increase of both the arithmetic and operator complexity (for the same target accuracy), adding additional challenges to the upscaling procedure. The number of 


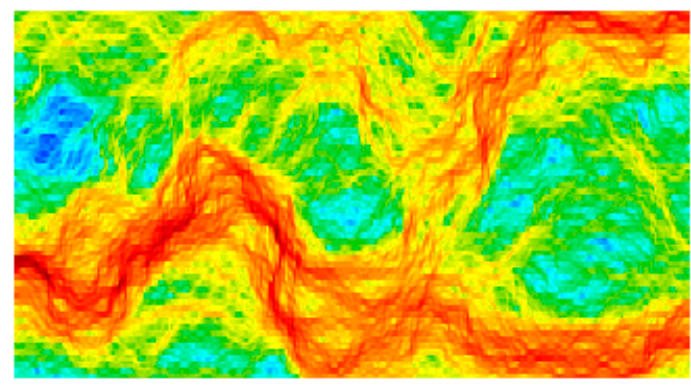

(a) Level 0

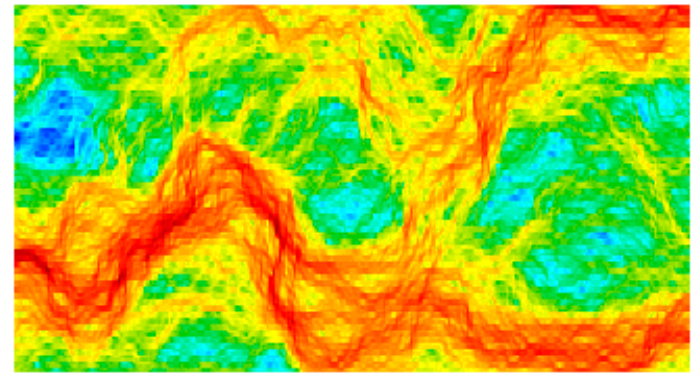

(c) Level 2

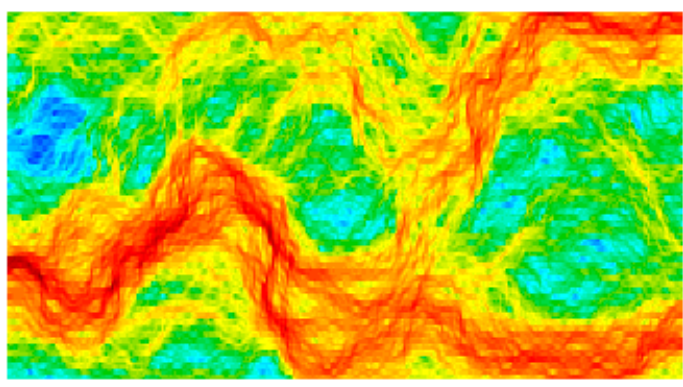

(b) Level 1

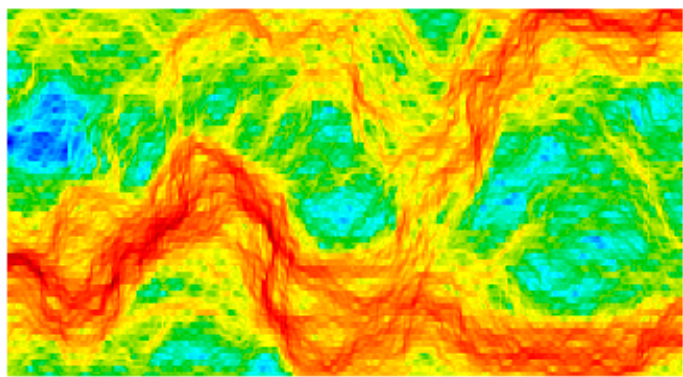

(d) Level 3

Figure 4.4: Velocity solutions on different levels in Experiment 4.

elements starting from level 0 to level 3 are 52800, 528, 10 and 2, respectively. The upscaling errors in different levels are shown in Table 4.6 The errors are slightly worse than case of structured hierarchy as expected. Nevertheless, the errors are generally still quite small.

\begin{tabular}{|c|c||c|c|c||c|c|}
\hline Level & $k$ & $\operatorname{dim}\left(\boldsymbol{V}_{H}\right)$ & $\operatorname{dim}\left(Q_{H}\right)$ & OC & $\frac{\left\|\boldsymbol{v}_{H}-\boldsymbol{v}_{h}\right\|}{\left\|\boldsymbol{v}_{h}\right\|}$ & $\frac{\left\|p_{H}-p_{h}\right\|}{\left\|p_{h}\right\|}$ \\
\hline 1 & 5 & 9282 & 2640 & 1.57 & 0.01669 & 0.00713 \\
2 & 5 & 195 & 50 & 1.58 & 0.03063 & 0.03237 \\
3 & 5 & 43 & 10 & 1.58 & 0.03063 & 0.03291 \\
\hline
\end{tabular}

Table 4.6: Performance of the $\rho$ AMGe upscaling in Experiment 5. For the fine-grid level, $\operatorname{dim}\left(\boldsymbol{V}_{h}\right)=106160$ and $\operatorname{dim}\left(Q_{h}\right)=52800$. 


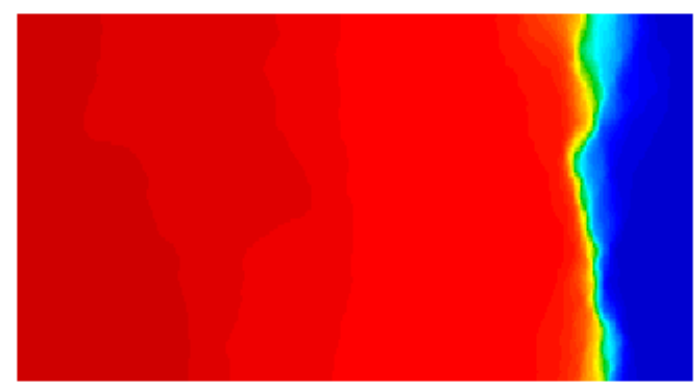

(a) Level 0

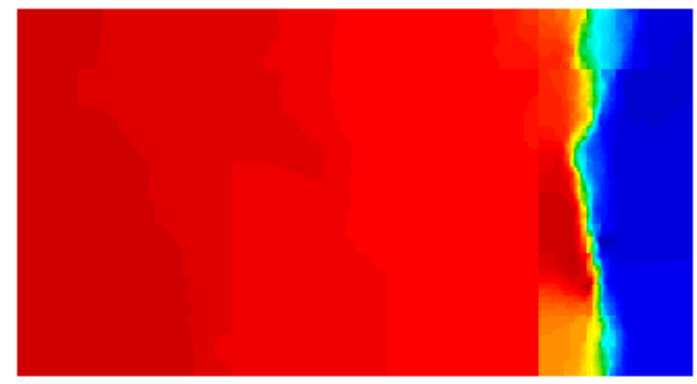

(c) Level 2

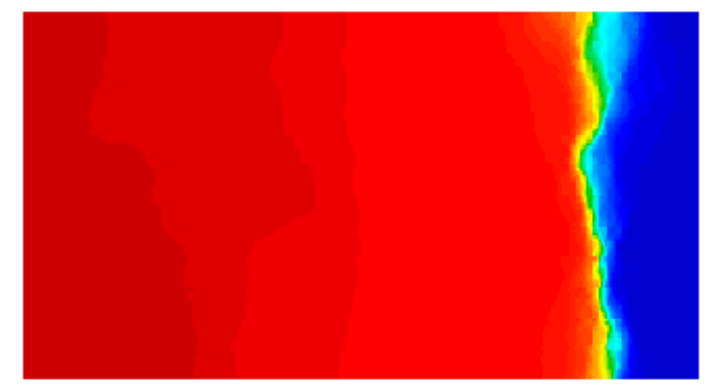

(b) Level 1

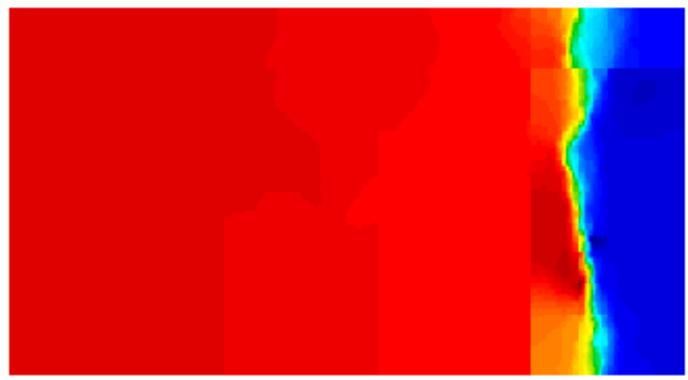

(d) Level 3

Figure 4.5: Pressure solutions on different levels in Experiment 4.
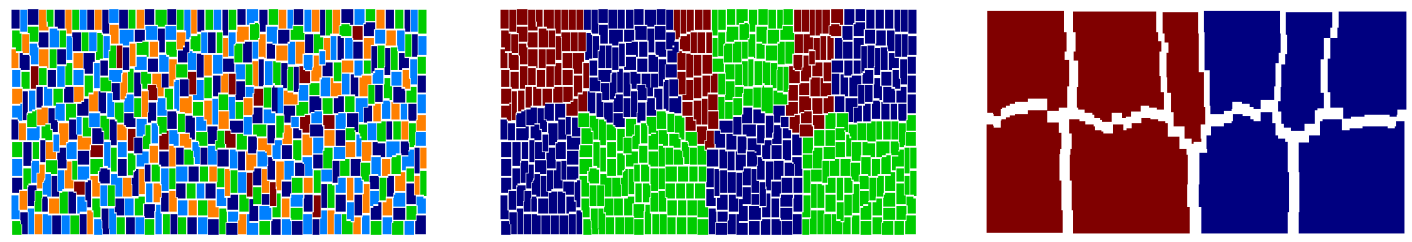

Figure 4.6: Agglomerates in each level in Experiment 4. To better visualize agglomerates we have applied a coloring algorithm. On level 2 (middle) and level 3 (right), each agglomerate consists of agglomerates from the previous (finer) level: we artificially separate the agglomerates coming from the previous level for visualization purposes.

Experiment 6: speedup by multilevel coarsening. We have claimed that a multilevel coarsening is faster than a two-level coarsening. In this experiment, we will verify that multilevel coarsening indeed provides a more efficient coarsening 
algorithm. The setup of this experiment is as follows. Starting with a fine grid of size $128 \times 192$, we will construct the coarse spaces by 2-, 3-, and 4-level hierarchies such that the coarsest grid is of size $2 \times 3$. In each level, we will take 4 local basis functions per coarse element/edge. The construction timings and upscaling errors for the coarsest spaces are shown in Table 4.7. We observe a huge saving in the

\begin{tabular}{|c|c||c|c|c|}
\hline nlevels & C.F. & C.S. Construction time & $\frac{\left\|v_{H}-v_{h}\right\|}{\left\|v_{h}\right\|}$ & $\frac{\left\|p_{H}-p_{h}\right\|}{\left\|p_{h}\right\|}$ \\
\hline 2 & $64 \times 64$ & $1463.49 \mathrm{~s}$ & 0.0163 & 0.0319 \\
3 & $8 \times 8$ & $11.29 \mathrm{~s}$ & 0.0257 & 0.0335 \\
4 & $4 \times 4$ & $9.58 \mathrm{~s}$ & 0.0304 & 0.0340 \\
\hline
\end{tabular}

Table 4.7: Coarsening timings and errors by 2-, 3-, and 4-level hierarchies.

construction time when using a 3 or 4-level hierarchy, which shows the success of the multilevel coarsening algorithm. On the other hand, the errors are more or less the same. Indeed, if we look at some of the basis functions (local eigenfunctions) constructed by different number of levels, the basis functions are very similar to each other, see Figure 4.7-4.8.

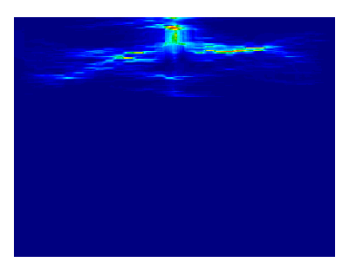

(a) 2-level construction

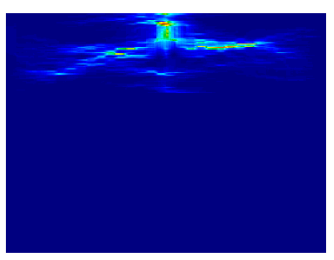

(b) 3-level construction

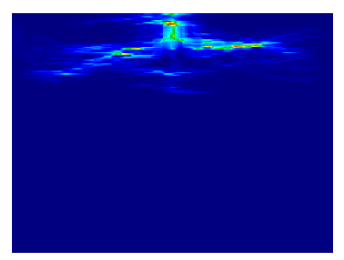

(c) 4-level construction

Figure 4.7: Velocity basis by different number of level constructions. 


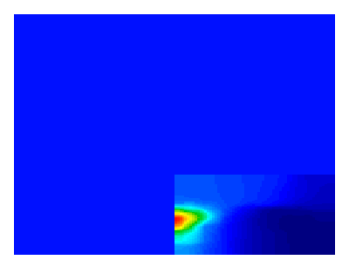

(a) 2-level construction

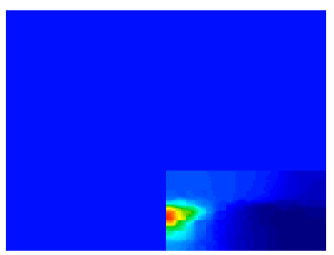

(b) 3-level construction

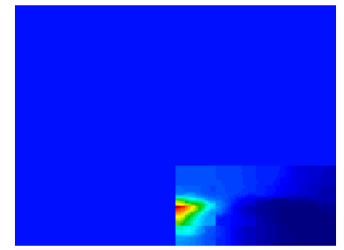

(c) 4-level construction

Figure 4.8: Pressure basis by different number of level constructions.

\subsubsection{D examples}

We consider here two 3D examples. The first one is the full SPE10 benchmark model, which contains high contrast channels and anisotropy, see Figure 4.1(b). The model is a relatively large one, and it is built on a structured Cartesian grid. For this model, the coarse spaces are built on a structured coarse grid. Since we will show the solving time for this model using a special solver that we propose, the full SPE10 example will be presented in the next section. The second test is the Egg model, [49, 48]. The name of the model comes from its shape being like an egg. It is obtained from a structured grid by setting some elements to be inactive. The resulting boundary of the model is irregular, so we build the coarse spaces on an unstructured coarse grid.

Experiment 7: the Egg model. The Egg model has more than 100 synthetic realizations of channelized reservoir permeability. In particular, we are using PERM18_ECL.INC, which can be downloaded from the model's official website [48]. The geometry and the Frobenuis norm of the permeability tensor are shown in Figure 4.9(a). The model contains 18553 elements, each of them of size $8 \times 8 \times 4$. 
The number of fine scale degrees of freedom is 59205 for the velocity and 18533 for the pressure. The coarse grid is obtained by using METIS to partition the fine grid into 128 agglomerates, see Figure 4.9(b). Table 4.8 shows the error behavior of the methods with respect to local spectral AMGe enrichment. We show the coarse and fine scale velocity solutions in Figure 4.10. We can see that the channelized feature is resolved gradually when more and more spectral basis functions are added to the local approximation spaces.

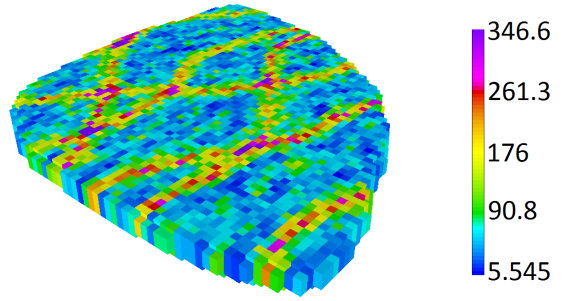

(a) Permeability field realization 18

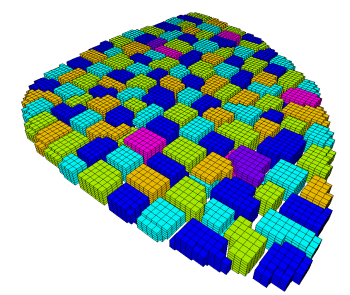

(b) Agglomeration by METIS

Figure 4.9: The Egg model.

\begin{tabular}{|c||c|c|c||c|c|}
\hline$k$ & $\operatorname{dim}\left(\boldsymbol{V}_{H}\right)$ & $\operatorname{dim}\left(Q_{H}\right)$ & OC & $\frac{\left\|\boldsymbol{v}_{H}-\boldsymbol{v}_{h}\right\|}{\left\|\boldsymbol{v}_{h}\right\|}$ & $\frac{\left\|p_{H}-p_{h}\right\|}{\left\|p_{h}\right\|}$ \\
\hline 2 & 1318 & 256 & 1.05 & 0.2546 & 0.05363 \\
3 & 2034 & 384 & 1.11 & 0.1801 & 0.03630 \\
4 & 2749 & 512 & 1.21 & 0.1452 & 0.03170 \\
5 & 3458 & 640 & 1.32 & 0.1123 & 0.02711 \\
6 & 4163 & 768 & 1.46 & 0.0811 & 0.01918 \\
\hline
\end{tabular}

Table 4.8: Performance of $\rho$ AMGe upscaling in Experiment 7. For the fine-grid level we have $\operatorname{dim}\left(\boldsymbol{V}_{h}\right)=59205$ and $\operatorname{dim}\left(Q_{h}\right)=18553$. 


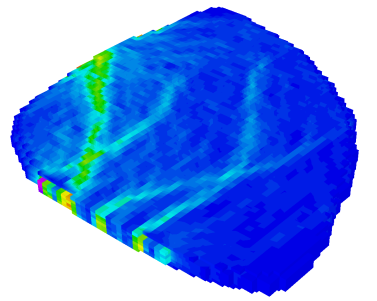

(a) Reference solution

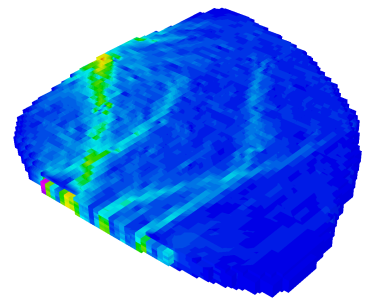

(b) Coarse solution: $k=2$

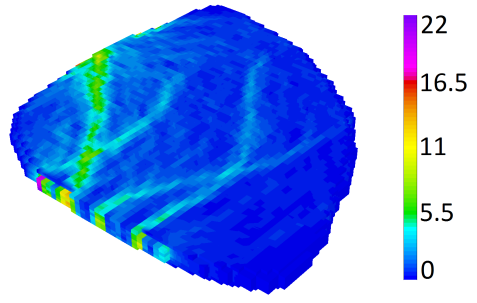

(c) Coarse solution: $k=6$

Figure 4.10: Magnitudes of velocity solutions in Experiment 6. 


\section{EFFICIENT SOLVER FOR COARSE SADDLE POINT SYSTEMS}

In practice, the coarse problem can still be too large to be solved by direct methods. Thus, efficient iterative methods for the coarse problem are desired. Due to the special shape functions in the coarse multiscale function space, some classical solver for the fine grid problems may not work well for the coarse problem. In fact, from our empirical results, some block diagonal preconditioners do not perform well for the coarse saddle point problems. In this section, we propose and test an efficient solver based on hybridization and AMG for the coarse problems. The solver is actually an efficient parallel algebraic solver for $\boldsymbol{H}$ (div) problems, see for example [59]. Therefore, our discussion will be concerned with the $\boldsymbol{H}$ (div) bilinear form acting on vector functions $\boldsymbol{u}, \boldsymbol{v}$ :

$$
a(\boldsymbol{u}, \boldsymbol{v})=\int_{\Omega} \alpha \nabla \cdot \boldsymbol{u} \nabla \cdot \boldsymbol{v}+\beta \boldsymbol{u} \cdot \boldsymbol{v} d \boldsymbol{x} .
$$

Here $\alpha, \beta \in L^{\infty}(\Omega)$ are some positive heterogeneous coefficients, and $\Omega$ is a simplyconnected polygonal domain in $\mathbb{R}^{d}, d=2,3$. Discrete problems associated with $a(\cdot, \cdot)$ arise in many applications, such as first order least squares formulation of second order elliptic problems [14], preconditioning of mixed finite element methods [12], Reissner-Mindlin plates [4] and the Brinkman equations [69]. Let $A$ be the linear system obtained from discretization of $a(\cdot, \cdot)$ by some $\boldsymbol{H}($ div)-conforming finite element of arbitrary order on a general unstructured mesh. Our goal is to design a 
scalable parallel solver for $A$.

It is well known that finding efficient iterative solvers for $A$ is not trivial because of the "near-null space" of $A$. The currently available scalable parallel solvers include the auxiliary space divergence solver (ADS) [55] in the hypre library and PCBDDC $[71,72]$ in the PETSc library. The former relies on the regular HX-decomposition for $\boldsymbol{H}$ (div) functions proposed in [44]. The setup of ADS is quite involved and require additional input from the user, namely, some discrete gradient and discrete curl operators. On the other hand, PCBDDC is based on the Balancing Domain Decomposition by Constraint algorithm [28]. Its construction requires that the local discrete systems are assembled at subdomain level. To accommodate high contrast and jumps in the coefficients, the primal space in PCBDDC is adaptively enriched by solving some generalized eigenvalue problems.

In this section, we propose an alternative way to solve systems with $A$. As an application, it can be used to efficiently solve the coarse saddle point system generated by the the mixed GMsFEM or AMGe. Our approach is based on traditional hybridization technique used in the mixed finite element method ([12]), thus reducing the problem to a smaller problem for the respective Lagrange multipliers that are involved in the hybridization. The reduced problem is symmetric positive definite, and as is well-known, is $H^{1}$-equivalent. Thus, in principle, one may apply any scalable AMG solver that is suitable for $H^{1}$ problems. Unlike ADS, the hybridization approach does not require additional information from the user. Instead, it requires 
that the original problem is given in unassembled element-based form.

One main issue that has to be addressed is the choice of the basis of the Lagrange multiplier space. In general, the reduced problem contains the constant function in its near null-space. However, if the basis for the Lagrange multipliers is not properly scaled (i.e., does not provide partition of unity), the coefficient vector of the constant functions is not a constant multiple of the vector of ones. The latter is a main assumption in the design of AMG for $H^{1}$-equivalent problems. We resolve this problem in an algebraic way by constructing a diagonal matrix which we use to rescale the reduced system such that the constant vector is the near-null space of the rescaled matrix, so that the respective AMG is correctly designed.

The proposed hybridization with diagonal rescaling is implemented in a parallel code and its scalability is tested in comparison with the state-of-the-art ADS solver. The results demonstrate that the new solver provides a competitive alternative to ADS; it clearly outperforms ADS for higher order elements [59]. As an important application of our interest, we will show its performance for coarse saddle point problems obtained from mixed type numerical upscaling/multiscale methods $[24,52]$. In particular, the solver can also be applied to solve coarse saddle point problems coming from coarsening of the graph Laplacian problems, which has application in upscaling of finite volume discretization in reservoir simulations, see [6] for a detail discussion. 


\subsection{Hybridization}

We consider the variational problem associated with the bilinear form (5.1): find $\boldsymbol{u} \in \boldsymbol{H}_{0}(\operatorname{div} ; \Omega)$ such that

$$
a(\boldsymbol{u}, \boldsymbol{v})=(f, \boldsymbol{v}), \quad \forall \boldsymbol{v} \in \boldsymbol{H}_{0}(\operatorname{div} ; \Omega)
$$

Here, $\boldsymbol{f}$ is a given function in $\left(L^{2}(\Omega)\right)^{d}$ and $(\cdot, \cdot)$ is the usual $L^{2}$ inner product in $\Omega$. Our following discussion is based on discretization of the variational problem (5.2) by Raviart-Thomas elements of arbitrary order. We note that other $\boldsymbol{H}$ (div)-conforming finite elements can also be considered. Let $\mathscr{T}_{h}$ be a general unstructured mesh on $\Omega$. The space of Raviart-Thomas elements of order $k \geq 0$ on $\mathscr{T}_{h}$ will be denoted by $R T_{k}$. For instance, if $\mathscr{T}_{h}$ is a simplicial mesh, then $R T_{k}$ is defined to be

$$
R T_{k}=\left\{\boldsymbol{v}_{h} \in \boldsymbol{H}_{0}(\operatorname{div} ; \Omega)\left|\boldsymbol{v}_{h}\right|_{\tau} \in\left(P_{k}(\tau)\right)^{d}+\boldsymbol{x} P_{k}(\tau) \quad \forall \tau \in \mathscr{T}_{h}\right\},
$$

where $P_{k}(\tau)$ denotes the set of polynomials of degree at most $k$ on $\tau$. For definitions of $R T_{k}$ on rectangular/cubic meshes, see for example [12]. Discretization of (5.2) by $R T_{k}$ elements results in a linear system of equations

$$
A u=f
$$

We are going to formulate an equivalent problem such that the modified problem can be solved more efficiently. We note that $R T_{k}$ basis functions are either associated with degrees of freedom (dofs) in the interior of elements, on boundary faces, or interior faces of a conforming finite element mesh. Those associated with dofs in the interior of elements or on boundary faces are supported in only one element, 
while those associated with dofs on interior faces are supported in two elements. In hybridization, the $R T_{k}$ basis functions that are associated with dofs on interior faces are split into two pieces, each supported in one and only one element. In practice, the splitting can be done by making use of the element-to-dofs relation table to identify the shared dofs between any pair of neighboring elements. This relation table can be constructed during the discretization. The space of Raviart-Thomas element after the splitting will be denoted by $\widehat{R T}_{k}$. If we discretize $a(\cdot, \cdot)$ with the basis functions in $\widehat{R T}_{k}$, the resulting system will have a block diagonal matrix $\widehat{A}$. Next, we need to enforce the continuity of the split basis functions in some way such that the solution of the modified system coincides with the original problem. Suppose a $R T_{k}$ basis function $\boldsymbol{\phi}$ is split into $\widehat{\boldsymbol{\phi}}_{1}$ and $\widehat{\boldsymbol{\phi}}_{2}$. The simplest way is to use Lagrange multiplier space to make the coefficient vectors of the test functions from both sides of an interior interface to be the same. If we set such constraints for all the split basis functions, we obtain a constraint matrix $C$.

Remark 5.1.1 There are other ways to enforce continuity of $\widehat{R T}_{k}$. For example, when constructing the constraint matrix $C$, one can also use the normal traces $\lambda$ of the original $R T_{k}$ basis functions as Lagrange multipliers, see [26].

The modified problem after introducing the Lagrange multipliers takes the saddlepoint form

$$
\left[\begin{array}{ll}
\widehat{A} & C^{T} \\
C & 0
\end{array}\right]\left[\begin{array}{l}
\widehat{u} \\
\lambda
\end{array}\right]=\left[\begin{array}{l}
\widehat{f} \\
0
\end{array}\right] .
$$


Here, $\widehat{u}$ is the coefficient vector of $\widehat{\boldsymbol{u}}_{h}$. The saddle point problem (5.4) can be reduced

$$
S \lambda=g
$$

where $S=C \widehat{A}^{-1} C^{T}$ and $g=C \widehat{A}^{-1} \widehat{f}$. The Schur complement $S$ and the new right hand side $g$ can be explicitly formed very efficiently because $\widehat{A}$ is block diagonal. In fact, the inversion of $\widehat{A}$ is embarrassingly parallel. Here, each local block of $\widehat{A}$ is invertible, so $\widehat{A}^{-1}$ is well-defined. We will show in the next section that $S$ is actually a s.p.d. system of the Lagrange multipliers, and that it can be solved efficiently by existing parallel linear solvers. After solving for $\lambda, \widehat{u}$ can be computed by back substitution $\widehat{u}=\widehat{A}^{-1}\left(\widehat{f}-C^{T} \lambda\right)$. Noticing that the back substitution involves only an action of $\widehat{A}^{-1}$ (already available in the computation of $S$ ) and some matrix-vector multiplications, which are inexpensive (local) and scalable computations.

\subsection{Discussion}

The hybridization approach described in the previous section can be summarized as follows

1. Split the $R T_{k}$ basis to obtain $\widehat{A}$ and $\widehat{f}$.

2. Compute $\widehat{A}^{-1}$ and form $S=C \widehat{A}^{-1} C^{T}$ and $g=C \widehat{A}^{-1} \widehat{f}$.

3. Solve the system $S \lambda=g$.

4. Recover $\widehat{u}$ by back substitution.

As explained in Section 5.1, step 2 and 4 are scalable (inexpensive local) computations. In contrast, step 3 involves the main computational cost. Thus, it is important 
that we can solve $S$ efficiently. In this section, we describe some properties of $S$. First, we show that $S$ is related to some hybridized mixed discretization of the second order differential operator $-\nabla \cdot\left(\beta^{-1} \nabla\right)+\alpha^{-1} I$ (acting on scalar functions). We note that the differential problem associated with (5.2) is

$$
-\nabla(\alpha \nabla \cdot \boldsymbol{u})+\beta \boldsymbol{u}=f
$$

with homogeneous Dirichlet boundary condition $\boldsymbol{u} \cdot \boldsymbol{n}=0$. The latter operator acts on vector-functions. We now make the following connection between these two operators. If we introduce an additional variable $p=\alpha \nabla \cdot \boldsymbol{u}$, then (5.6) becomes the following first order system (for $\boldsymbol{u}$ and $p$ )

$$
\begin{aligned}
\beta \boldsymbol{u}-\nabla p & =\boldsymbol{f}, \\
\nabla \cdot \boldsymbol{u}-\alpha^{-1} p & =0 .
\end{aligned}
$$

It is noteworthy to note that the structure of $(5.7)$ is the same as the mixed formulation of the differential operator $-\nabla \cdot\left(\beta^{-1} \nabla\right)+\alpha^{-1} I$. So we can apply a hybridized mixed discretization $[26,27]$ for $-\nabla \cdot\left(\beta^{-1} \nabla\right)+\alpha^{-1} I$ to discretize (5.7). To apply the the hybridized mixed discretization, we note that the weak form of (5.7) is to find $(\boldsymbol{u}, p) \in \boldsymbol{H}_{0}(\operatorname{div} ; \Omega) \times L^{2}(\Omega)$ such that

$$
\begin{aligned}
(\beta \boldsymbol{u}, \boldsymbol{v})+(p, \nabla \cdot \boldsymbol{v}) & =(\boldsymbol{f}, \boldsymbol{v}) & & \forall \boldsymbol{v} \in \boldsymbol{H}_{0}(\operatorname{div} ; \Omega) \\
(\nabla \cdot \boldsymbol{u}, q)-\left(\alpha^{-1} p, q\right) & =0 & & \forall q \in L^{2}(\Omega) .
\end{aligned}
$$

Let $W_{h}^{k} \subset L^{2}(\Omega)$ be a space of piecewise polynomials such that $R T_{k}$ and $W_{h}^{k}$ form a stable pair for the mixed discretization of (5.8). For instance, for simplicial meshes, 
we can take

$$
W_{h}^{k}=\left\{q \in L^{2}(\Omega)|q|_{\tau} \in P_{k}(\tau) \quad \forall \tau \in \mathscr{T}_{h}\right\}
$$

If (5.8) is discretized by the pair $\widehat{R T}_{k}-W_{h}^{k}$ and the continuity of $\widehat{R T}_{k}$ is enforced by the constraint matrix $C$ as described in Section 5.1, we get a 3 by 3 block system of equations of the form

$$
\left[\begin{array}{ccc}
\widehat{M} & \widehat{B}^{T} & C^{T} \\
\widehat{B} & -W & 0 \\
C & 0 & 0
\end{array}\right]\left[\begin{array}{l}
\widehat{u} \\
p \\
\lambda
\end{array}\right]=\left[\begin{array}{l}
\widehat{f} \\
0 \\
0
\end{array}\right]
$$

As $\widehat{M}$ and $W$ are weighted $L^{2}$ mass matrices of the spaces $\widehat{R T}_{k}$ and $W_{h}^{k}$ respectively, they are invertible. Hence, the 2 by 2 block matrix $\left[\begin{array}{cc}\widehat{M} & \widehat{B}^{T} \\ \widehat{B} & -W\end{array}\right]$ is invertible, and (5.9) can be reduced to

$$
\left[\begin{array}{ll}
C & 0
\end{array}\right]\left[\begin{array}{cc}
\widehat{M} & \widehat{B}^{T} \\
\widehat{B} & -W
\end{array}\right]^{-1}\left[\begin{array}{l}
C^{T} \\
0
\end{array}\right] \lambda=\left[\begin{array}{ll}
C & 0
\end{array}\right]\left[\begin{array}{cc}
\widehat{M} & \widehat{B}^{T} \\
\widehat{B} & -W
\end{array}\right]^{-1}\left[\begin{array}{l}
\widehat{f} \\
0
\end{array}\right]
$$

Since the $(1,1)$ block of $\left[\begin{array}{cc}\widehat{M} & \widehat{B}^{T} \\ \widehat{B} & -W\end{array}\right]^{-1}$ can be written as $\left(\widehat{M}+\widehat{B}^{T} W^{-1} \widehat{B}\right)^{-1}$ and $\widehat{A}=\widehat{M}+\widehat{B}^{T} W^{-1} \widehat{B}$, the reduced problem (5.10) is in fact identical to (5.5). Therefore, the Schur complement $S$ in (5.5) can be characterized by the hybridized mixed discretization for the differential operator $-\nabla \cdot\left(\beta^{-1} \nabla\right)+\alpha^{-1} I$.

Remark 5.2.1 Actually the hybridized mixed discretization for $-\nabla \cdot\left(\beta^{-1} \nabla\right)+\alpha^{-1} I$ 
in $[26,27]$ gives rise to the reduced system $\widetilde{S}$ for the Lagrange multiplier $\lambda$ where

$$
\widetilde{S}=C\left(\widehat{M}^{-1}-\widehat{M}^{-1} \widehat{B}^{T}\left(\widehat{B} \widehat{M}^{-1} \widehat{B}^{T}+W\right)^{-1} \widehat{B} \widehat{M}^{-1}\right) C^{T}
$$

However, since $W$ is invertible, an application of the Sherman-Morrison-Woodbury formula implies that $\widetilde{S}=S$.

In [27], the authors proved that $S$ is spectrally equivalent to the norm $\|\cdot\| \cdot \|$ on the space of Lagrange multipliers defined as

$$
\|\lambda\|^{2}=\sum_{\tau \in \mathscr{T}_{h}} \frac{1}{|\partial \tau|}\left\|\lambda-m_{\tau}(\lambda)\right\|_{\partial \tau}^{2}
$$

where $m_{\tau}(\lambda)=\frac{1}{|\partial \tau|} \int_{\partial \tau} \lambda d s$. More precisely, there are constants $C_{1}$ and $C_{2}$, depending only on the approximation order $k$, the coefficients $\alpha, \beta$ of the operator, and the shape regularity of $\mathscr{T}_{h}$ such that

$$
C_{1}\|\lambda\|^{2} \leq \lambda^{T} S \lambda \leq C_{2}\|\lambda\|^{2} \quad \forall \lambda
$$

Consequently, $S$ is symmetric positive definite. Moreover, this shows that the nearnull space of $S$ is spanned by the constant functions, which is the main assumption to successfully apply solvers of AMG type. When solving with $S$, we opt for the parallel algebraic multigrid solver BoomerAMG from the hypre library [43].

The fact that the constant functions are in the near-null space of $S$ is not sufficient to guarantee the efficiency of BoomerAMG. One of the factors affecting the success of BoomerAMG is that the constant coefficient vector $\mathbf{1}$ should be in the near-null space of $S$ (as a matrix). However, this is not always the case. Indeed, depending on the choice of basis for the Lagrange multipliers space, the coefficient 
vector of a constant function is not necessarily a constant vector. To resolve this issue, we chose to rescale $S$ by a diagonal matrix $D$ such that the constant vector is now in the near-null space of $D^{T} S D$. To achieve this, we solve the homogeneous problem $S d=0$ by applying a few smoothing steps to a random initial guess. In our numerical experiments to be presented in the next section, we use 5 conjugate gradient $(\mathrm{CG})$ iterations preconditioned by the Jacobi smoother in the computation of $d$, which is fairly inexpensive. Once $d$ is computed, we set $D_{i i}=d_{i}$ (the $i$ th entry of d). Noticing that $D \mathbf{1}=d$, so $\mathbf{1}$ is in the near-null space of $D^{T} S D$. We can then apply CG preconditioned by BoomerAMG constructed from $D^{T} S D$ to efficiently solve the system

$$
\left(D^{T} S D\right) \lambda_{D}=D^{T} g
$$

Lastly, the original Lagrange multiplier $\lambda$ is recovered simply by setting $\lambda=D \lambda_{D}$.

Another useful feature of $S$ is that its size is less than or equal to the size of the original matrix $A$. This is because there is a one-to-one correspondence between Lagrange multipliers and Raviart-Thomas basis functions associated with interior faces. For higher order Raviart-Thomas elements, a portion of the basis functions are associated with interior of elements. These basis functions are supported in one element only, so they do not need Lagrange multipliers to enforce their continuity. Hence, for higher order approximations, the size of $S$ is considerably smaller than the size of $A$. As a result, methods for solving with $S$ is likely to be more efficient and faster than solving with $A$ (using the state-of-the-art solvers such as ADS) which is 
confirmed by our experiments.

\subsection{Numerical examples}

In this section, we present some numerical results regarding the performance of our hybridization AMG solver. All the experiments are performed on the cluster Sierra at the Lawrence Livermore National Laboratory. Sierra has a total of 1944 nodes (Intel Xeon EP X5660 clocked at $2.80 \mathrm{GHz}$ ), which are connected by InfiniBand QDR. Each node has 12 cores and equipped with 24 GB of memory.

In the solution process, the hybridized system with $S$ is rescaled by the diagonal matrix $D$ as described in the previous section. The rescaled system $D^{T} S D$ is then solved by the CG method preconditioned with BoomerAMG (constructed from $D^{T} S D$ ) from the hypre library. As one of our goals is to compare the hybridization AMG solver with ADS, we present also the performance of ADS in all the examples. In order to have fair comparisons, the time to solution for the hybridization AMG solver includes the formation time of the Schur complement $S$, the computation time to construct the rescaling matrix $D$, the solve time for the problem with the modified matrix $D^{T} S D$ by CG preconditioned by BoomerAMG, and the recovery time of the original unknown $\boldsymbol{u}$. The time to solution for ADS is simply the solve time for the original problem with $A$ by the CG preconditioned by ADS. For the tables in the present section, \# proc refers to the number of processors, while \# iter refers to the number of PCG iterations. 


\subsubsection{Weak scaling}

We first test the weak scaling of the hybridization AMG solver. The problem setting is as follows. We will solve problem (5.3) obtained by $R T_{k}$ discretization on uniform tetrahedral mesh in 3D. Starting from some initial tetrahedral mesh, we refine the mesh uniformly. The problem size will be increased by about 8 times after one such refinement. At the same time, the number of processors for solving the refined problem is increased 8 times so that the problem size per processor is kept roughly the same. Both the lowest order Raviart-Thomas elements $R T_{0}$ and a higher

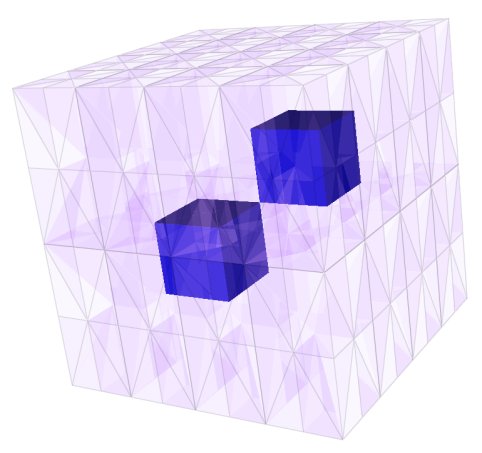

Figure 5.1: Initial mesh for the $R T_{2}$ weak scaling test case. Blue region indicates $\Omega_{i}$.

order elements, $R T_{2}$, are considered. We solve a heterogeneous coefficient problem on the unit cube, i.e. $\Omega=[0,1]^{3}$. The boundary conditions are $\boldsymbol{u} \cdot \boldsymbol{n}=0$ on $\partial \Omega$, and the source function $f$ is the constant vector $[1,1,1]^{T}$. Let $\Omega_{i}=\left[\frac{1}{4}, \frac{1}{2}\right]^{3} \cup\left[\frac{1}{2}, \frac{3}{4}\right]^{3}$. We 
will consider $\beta$ being constant 1 throughout the domain, whereas

$$
\alpha= \begin{cases}1 & \text { in } \Omega \backslash \Omega_{i} \\ 10^{p} & \text { in } \Omega_{i}\end{cases}
$$

and we choose $p=-4,0$, or 4 . For $R T_{2}$ test case, we first partition $\Omega$ into $8 \times 8$ x 4 parallelepipeds. The initial tetrahedral mesh in this case is then obtained by subdividing each parallelepiped into tetrahedrons, see Figure 5.1. The initial mesh of the $R T_{0}$ test case is obtained by refining the initial mesh of the $R T_{2}$ test case 3 times. The PCG iterations are stopped when the $l_{2}$ norm of the residual is reduced by a factor of $10^{10}$. The time to solution (in seconds) of both the hybridization AMG and ADS for the $R T_{0}$ case are shown in Table 5.1. Additionally, we also report the number of PCG iterations in the brackets. We see that the number of iterations of

\begin{tabular}{|c|c||c|c|c|}
\hline \multicolumn{2}{|c||}{$\alpha=\left\{1,10^{p}\right\}, \beta \equiv 1$} & \multicolumn{3}{c|}{$p$} \\
\hline \# proc & Problem size & -4 & 0 & 4 \\
\hline \multicolumn{5}{|c|}{ Hybridization-BoomerAMG-CG } \\
\hline 3 & 200,704 & $0.97(24)$ & $0.96(21)$ & $0.93(21)$ \\
24 & $1,589,248$ & $1.15(24)$ & $1.15(23)$ & $1.16(23)$ \\
192 & $12,648,448$ & $1.45(27)$ & $1.48(25)$ & $1.43(24)$ \\
1,536 & $100,925,440$ & $3.31(29)$ & $3.03(28)$ & $3.03(28)$ \\
\hline \multicolumn{5}{|c|}{ ADS-CG } \\
\hline 3 & 200,704 & $2.68(21)$ & $1.74(10)$ & $1.79(11)$ \\
24 & $1,589,248$ & $4.04(25)$ & $3.53(13)$ & $3.54(13)$ \\
192 & $12,648,448$ & $7.10(27)$ & $5.73(15)$ & $5.61(14)$ \\
1,536 & $100,925,440$ & $8.30(28)$ & $6.28(15)$ & $6.51(15)$ \\
\hline
\end{tabular}

Table 5.1: Time to solution in seconds: $R T_{0}$ on tetrahedral meshes, the corresponding number of PCG iterations are the reported in the brackets.

the hybridization solver are very stable against problem size and the heterogeneity 
of $\alpha$. The average time to solution of the hybridization approach is about 2 times faster than that of ADS. The solution time difference between the two solvers is more significant in the high order discretization case. This is due to the fact that size of the hybridized system $S$ is much smaller than the size of the original system $A$. Indeed, in the case of $R T_{2}$, the average time to solution of the hybridization approach is about 8 times faster than that of ADS, see Table 5.2. In Figure 5.2, we plot the solution time of both solvers where $p=4$ in the definition of $\alpha$. We can see that the hybridization solver has promising weak scaling up to 1536 processors.

\begin{tabular}{|c|c||c|c|c|}
\hline \multicolumn{2}{|c||}{$\alpha=\left\{1,10^{p}\right\}, \beta \equiv 1$} & \multicolumn{3}{c|}{$p$} \\
\hline \# proc & Problem size & -4 & 0 & 4 \\
\hline \multicolumn{5}{|c|}{ Hybridization-BoomerAMG-CG } \\
\hline 3 & 38,400 & $0.30(15)$ & $0.31(16)$ & $0.31(16)$ \\
24 & 301,056 & $0.48(18)$ & $0.50(21)$ & $0.48(20)$ \\
192 & $2,383,872$ & $0.75(28)$ & $0.89(29)$ & $0.77(29)$ \\
1,536 & $18,972,672$ & $1.97(44)$ & $1.95(47)$ & $2.10(47)$ \\
\hline \multicolumn{5}{|c|}{ ADS-CG } \\
\hline 3 & 38,400 & $4.85(23)$ & $3.55(13)$ & $3.80(14)$ \\
24 & 301,056 & $7.24(29)$ & $5.47(18)$ & $5.73(20)$ \\
192 & $2,383,872$ & $11.56(37)$ & $8.89(25)$ & $9.56(28)$ \\
1,536 & $18,972,672$ & $24.28(53)$ & $16.51(37)$ & $16.37(39)$ \\
\hline
\end{tabular}

Table 5.2: Time to solution in seconds: $R T_{2}$ on tetrahedral meshes, the corresponding number of PCG iterations are the reported in the brackets.

\subsubsection{Strong scaling}

In the second example, we investigate the strong scaling of the hybridization AMG solver. The problem considered in this section is the crooked pipe problem, see 


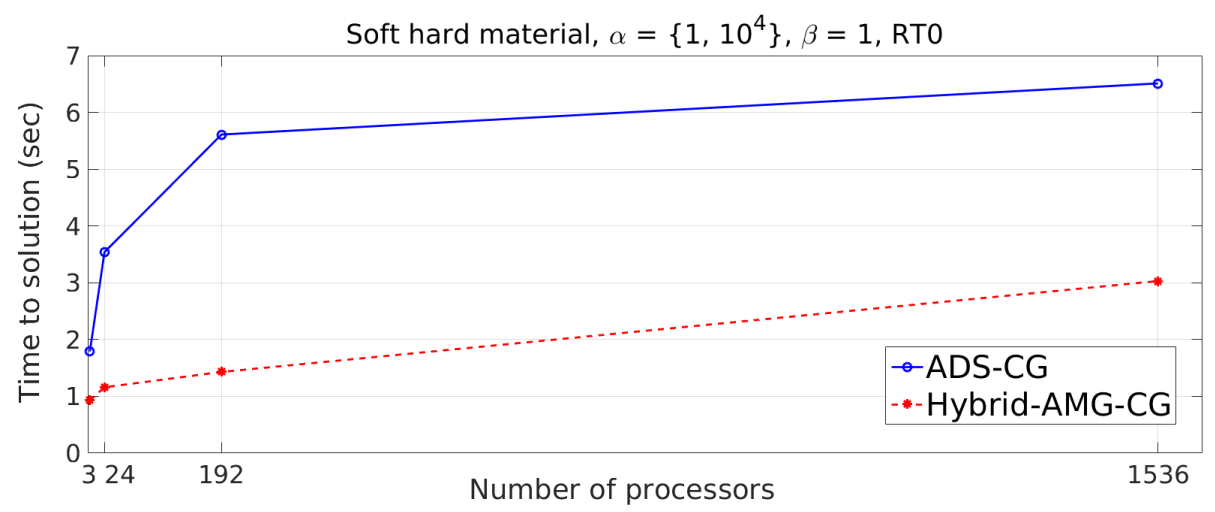

(a) $R T_{0}$

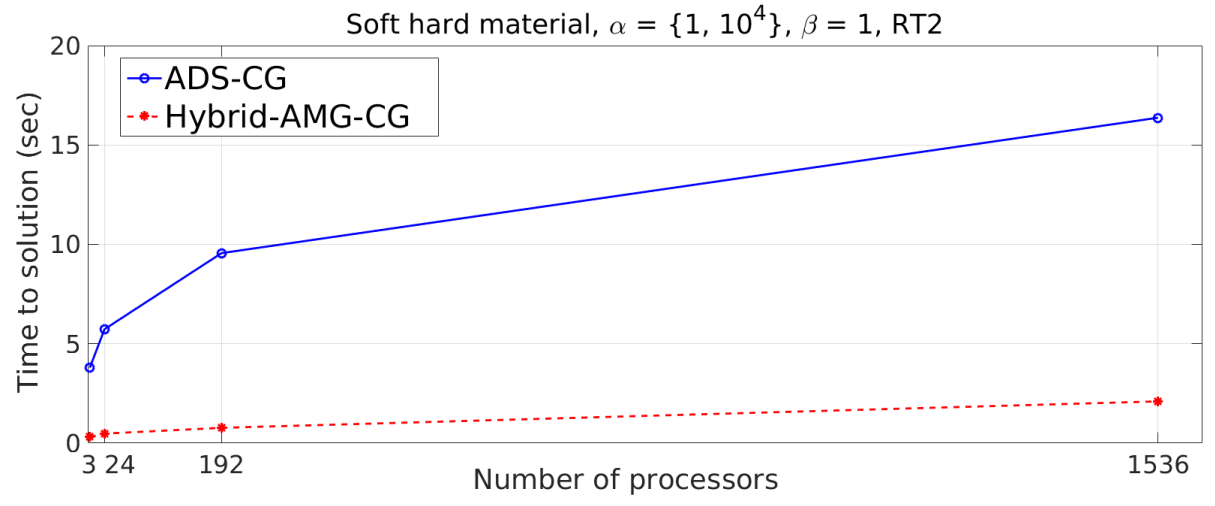

(b) $R T_{2}$

Figure 5.2: Weak scaling comparisons between hybridization (red dotted line) and ADS (blue solid line)

[55] for a detail description of the problem. The mesh for this problem is depicted in Figure 5.3. The coefficient $\alpha$ and $\beta$ are piecewise constants. More precisely, $(\alpha, \beta)=(1.641,0.2)$ in the red region, and $(\alpha, \beta)=(0.00188,2000)$ in the blue region. The difficulties of this problem are the large jumps of coefficients and the highly stretched elements in the mesh (see Figure 5.3). For this test, the problem discretized by $R T_{1}$. The size of $A$ is $2,805,520$, and we solve the problem using 4, 8, 16,32 and 64 processors. The PCG iteration is stopped when the $l_{2}$ norm of the residual is 

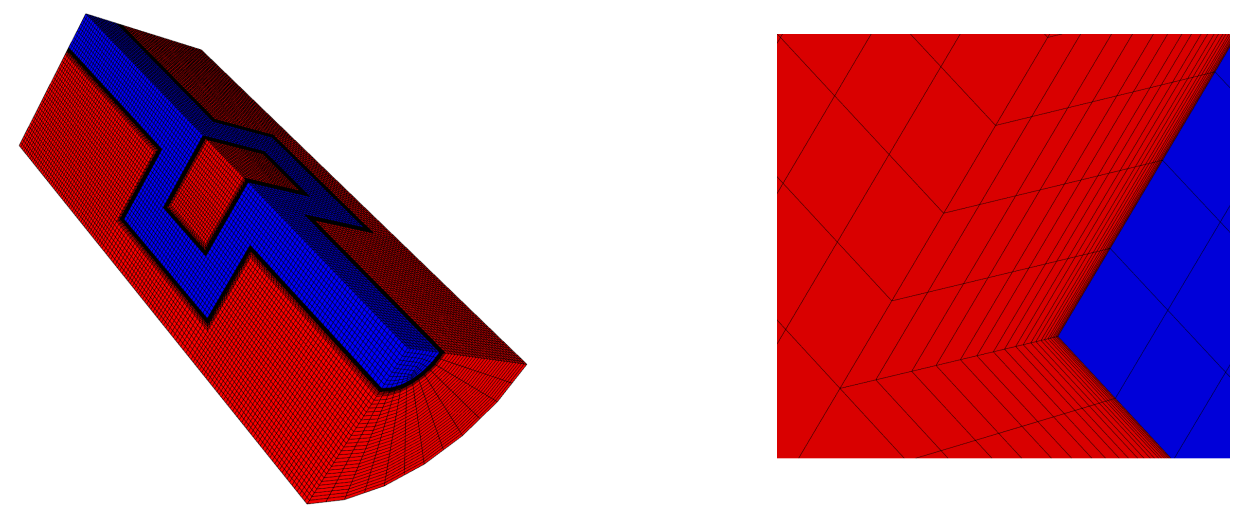

Figure 5.3: The mesh for the Crooked Pipe problem (left). A dense layer of highly stretched elements (right) has been added to the neighborhood of the material interface in the exterior subdomain in order to resolve the physical diffusion.

reduced by a factor of $10^{14}$. The number of PCG iterations and time to solution are reported in Table 5.3, and we plot the speedup in Figure 5.4. When measuring the speedup, solution time are corrected by the number of iterations.

Both solvers exhibit good strong scaling. We note that in this example, the solution time of the hybridization AMG solver is much smaller than the ADS solver. The average solve time of the hybridization AMG solver is about 10 times smaller than that of ADS. In particular, the hybridization AMG solver with 4 processors is still 2 times faster than ADS with 64 processors. The difference in the computation time for this example is highly noticeable.

Lastly, we report the time spent on different components of the hybridization approach in Table 5.4. We observe that except solving with $S$ (i.e. setup and PCG solve), the other components scale fairly well. Also, as we point out in Section 5.2, solving with $S$ is the most time consuming part of the hybridization AMG code. We 


\begin{tabular}{|c||c|c||c|c|}
\hline \multicolumn{1}{|c||}{} & \multicolumn{2}{c||}{ Hybridization-BoomerAMG-CG } & \multicolumn{2}{c|}{ ADS-CG } \\
\hline \# proc & \# iter & time to solution & \# iter & time to solution \\
\hline 4 & 25 & 23.46 & 32 & 508.66 \\
8 & 31 & 14.21 & 32 & 251.37 \\
16 & 28 & 6.83 & 33 & 130.26 \\
32 & 28 & 3.98 & 34 & 73.47 \\
64 & 31 & 2.92 & 34 & 54.58 \\
\hline
\end{tabular}

Table 5.3: Strong scaling test, original problem size: 2,805,520.

remark that during the formation of $S$, we stored the inverses of local blocks of $\widehat{A}$. So when we recover $u$ by back substitution, only matrix multiplication is needed. Hence, the recovery of $u$ is extremely cheap and scalable.

\begin{tabular}{|c||c|c|c|c|c|}
\hline \# proc & formation of $S$ & computation of $D$ & setup & PCG solve & recovery of $u$ \\
\hline 4 & 7.55 & 0.22 & 3.87 & 11.72 & 0.092 \\
8 & 3.95 & 0.11 & 2.29 & 7.81 & 0.046 \\
16 & 1.84 & 0.057 & 1.4 & 3.52 & 0.022 \\
32 & 1.11 & 0.034 & 0.83 & 2.01 & 0.012 \\
64 & 0.68 & 0.027 & 0.52 & 1.7 & 0.006 \\
\hline
\end{tabular}

Table 5.4: Timing of each component of the new solver.

\subsubsection{Coarse saddle point problems}

Although the coarse system coming from numerical upscaling/multiscale methods has a much smaller dimension than the fine grid problem, in general it is not small enough that one can simply solve it using a direct solver. We recall that the coarse system from mixed GMsFEM/ $\rho$ AMGe has also a saddle-point form,

$$
\mathscr{A}_{H}=P^{T} \mathscr{A}_{h} P=\left[\begin{array}{cc}
M_{H} & B_{H}^{T} \\
B_{H} & 0
\end{array}\right] .
$$




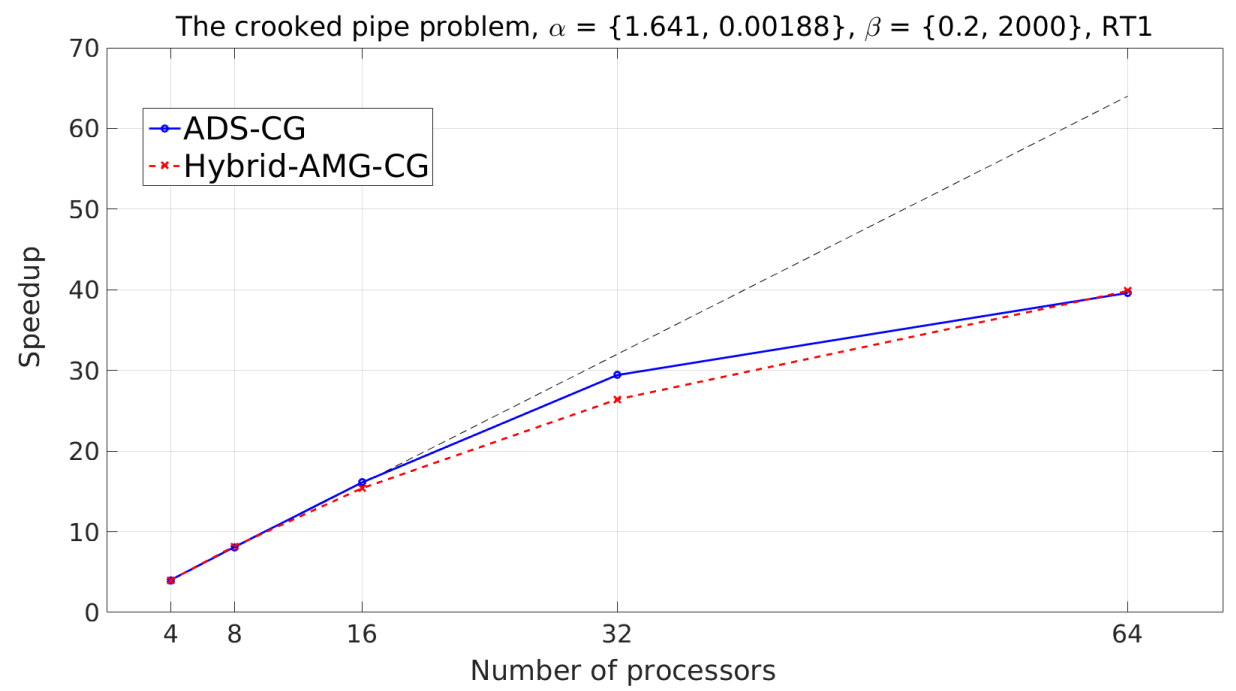

Figure 5.4: Strong scaling comparison between hybridization (red dotted line) and ADS (blue solid line). Black dotted line indicates perfect scaling

Note that due to the somewhat complicated structure of the basis of $\boldsymbol{V}_{H}$ (we have more than one dof per coarse face $F$ and possibly multiple dofs per coarse element for the pressure space $Q_{H}$ ), standard solvers like block-diagonal preconditioners such as $L^{2}-H^{1}$-equivalent, or $H(\mathrm{div})-L^{2}$-equivalent (for terminology and details, cf., [61]), are not directly applicable in an "out of shelf" manner. For that reason, we decided instead of solving the saddle point problem (5.11) directly, to solve a reduced symmetric positive definite system obtained by hybridization of (5.11). This is possible due to the fact that mixed GMsFEM/ $\rho$ AMGe has the same properties as a traditional finite element discretization approach, however with non-standard elements. In particular, the global coarse problem can be assembled from local element matrices, and also the coarse elements have well-defined topology, in particular, their boundary is split into well-defined faces $F$. Then, the hybridization approach, well- 
known for finite elements (cf. [63]), applies in a straightforward manner to the coarse saddle point problems.

We will test the proposed solver for solving the coarse problems obtained by coarsening the full SPE10 benchmark model using $\rho$ AMGe. The fine grid is a $60 \times 220 \times 85$ structured Cartesian grid, with the size of each element being $20 \mathrm{ft} \times 10 \mathrm{ft} \times 2 \mathrm{ft}$. The number of fine scale degrees of freedom for the velocity is $3,403,000$, while that for the pressure is $1,122,000$. The permeability field $\kappa$ is anisotropic (especially in the $z$-direction). Convergence of $\rho$ AMGe against local spectral enrichment will also be shown for this 3D model. The coarse grid is fixed to be $6 \times 11 \times 29$, and we enrich the local approximation spaces gradually by increasing the number of local basis functions. The convergence (depending on number of local basis functions) is evident from Table 5.5.

The experiments in Table 5.5 were computed on an Intel Xeon processor X5660 (clocked at $2.8 \mathrm{GHz}$ ) with 24 GByte memory. The fine-grid problem was solved using the minimal residual method preconditioned by a state-of-the-art $L^{2}-H^{1}$ block diagonal preconditioner, which for this specific problem appeared to be the fastest of the solvers we tested ( $H($ div $)-L^{2}$ block diagonal preconditioner and hybridization). The solution time for the fine scale problem is roughly 61 seconds. On the other hand, the coarse problem is solved by the proposed solver based on hybridization and AMG. However, instead of BoomerAMG, we use the $\rho$ AMGe solver developed for $H^{1}$ problems to solve the reduced system after hybridization. We observe, that 
if the operator complexity of the coarse problem is about 1.02, the solution time for the coarse problem is less than 0.5 seconds, which means the speedup is more than 100 times (versus the fine-grid problem). When the operator complexity is around 1.32, then the solution time for the coarse problem increases to about 14 seconds, so the speedup reduces to roughly 4 times. We remark here that if we apply $L^{2}-H^{1}$ block diagonal preconditioner and solve the coarse saddle point problem by minimal residual method, the number of iterations can easily go up to more than 1000 .

\begin{tabular}{|c||c|c|c|c|c|c|}
\hline$k$ & $\operatorname{dim}\left(\boldsymbol{V}_{H}\right)$ & $\operatorname{dim}\left(P_{H}\right)$ & OC & PCG time & $\frac{\left\|v_{H}-\boldsymbol{v}_{h}\right\|}{\left\|v_{h}\right\|}$ & $\frac{\left\|p_{H}-p_{h}\right\|}{\left\|p_{h}\right\|}$ \\
\hline 3 & 22731 & 5742 & 1.02 & $0.35 \mathrm{~s}(21)$ & 0.4398 & 0.05377 \\
6 & 47376 & 11484 & 1.08 & $2.27 \mathrm{~s}(33)$ & 0.3241 & 0.04188 \\
9 & 72021 & 17226 & 1.18 & $7.19 \mathrm{~s}(49)$ & 0.26436 & 0.03759 \\
12 & 96522 & 22968 & 1.32 & $14.3 \mathrm{~s}(55)$ & 0.2230 & 0.03467 \\
\hline
\end{tabular}

Table 5.5: The numbers in the bracket is the CG iteration count. For the fine-grid level, $\operatorname{dim}\left(\boldsymbol{V}_{h}\right)=3,403,000$ and $\operatorname{dim}\left(Q_{h}\right)=1,122,000$. The solution time for the fine scale problem is about 61 seconds. 


\section{CONCLUSION}

In this dissertation, two enrichment schemes for mixed multiscale finite element methods are studied. As explained in Section 1, the study is motivated by the fact that mixed multiscale finite element methods are inadequate to provide accurate coarse scale approximations for complex real world problems.

The first enrichment scheme, mixed GMsFEM, is based on the GMsFEM framework. The original GMsFEM was proposed for second order elliptic problems in primal form. Although GMsFEM does provide a increased accuracy, the resulting solution is not mass conservative. In contrast, the solution of the mixed GMsFEM automatically conserves mass on the coarse grid. If necessary, an inexpensive local postprocessing procedure can be applied to produce a mass conservative solution on the fine grid.

The mixed GMsFEM for Darcy's flow and linear elasticity are proposed. For Darcy's flow, direct comparison can be made to the mixed multiscale finite element methods. In fact, the design of mixed GMsFEM is inspired by the mixed multiscale finite element methods for Darcy's flow. Selection of local spectral problems are based on analysis. The mixed GMsFEM is then extended to solve linear elasticity. However, the extension is not trivial due to the symmetry requirement of the stress tensor. Indeed, such a requirement implicitly forces the coarse approximation space for displacement to include all the rigid motions in each coarse elements. Moreover, 
the requirement also leads to a decomposition of the coarse approximation space of the stress tensor into a edge-based subspace and a vertex-based subspace. Discretization of the mixed formulation of the linear elasticity system is well-known to be complicated; the current algorithm is applicable in 2D. Further research is needed for elasticity problems in 3D.

The second enrichment scheme is based on $\rho \mathrm{AMGe}$, where both the velocity and pressure coarse spaces are enriched. Due to the multigrid nature, recursive application of the algorithm is readily available, which renders the algorithm a multilevel method. Our numerical results show that the multilevel method can lead to a significant saving in the coarse space construction time, while the accuracy are maintained.

In practice, the coarse problem can still be too large to be solved by direct methods. So efficient iterative methods for the coarse problem are desired. Due to the special shape functions in the coarse multiscale function space, some classical solver for the fine grid problems may not work well for the coarse problem. In fact, from our empirical results, some block diagonal preconditioners do not perform well for the coarse problems. We proposed and tested an efficient solver for the coarse saddle point problems based on hybridization and AMG. While ADS requires the user to provide some discrete curl and gradient operators, our proposed solver does not require extra input. Hence, it is applicable to more general problems. The solver shows comparable scalability to ADS, a state-of-the-art solver for $H$ (div) problems. 
Moreover, It outperforms ADS in terms of CPU solving time.

For all the proposed multscale methods, stability of the coarse systems as well as convergence analysis are discussed. Furthermore, extensive sets of experiments in $2 \mathrm{D}$ and $3 \mathrm{D}$ are conducted to numerically verify the validation of the proposed schemes. In particular, application in speeding up single- and two-phase flow simulations is demonstrated. Robustness of the mixed GMsFEM for modeling mixture of compressible and nearly incompressible materials are also illustrated. 


\section{REFERENCES}

[1] J. E. Aarnes. On the use of a mixed multiscale finite element method for greater flexibility and increased speed or improved accuracy in reservoir simulation. Multiscale Modeling \& Simulation, 2:421-439, 2004.

[2] T. Arbogast and K. J. Boyd. Subgrid upscaling and mixed multiscale finite elements. SIAM Journal on Numerical Analysis, 44(3):1150-1171, 2006.

[3] T. Arbogast, G. Pencheva, M. F. Wheeler, and I. Yotov. A multiscale mortar mixed finite element method. Multiscale Modeling \& Simulation, 6(1):319-346, 2007.

[4] D. N. Arnold, R. S. Falk, and R. Winther. Preconditioning discrete approximations of the Reissner-Mindlin plate model. RAIRO Modél. Math. Anal. Numér., 31(4):517-557, 1997.

[5] I. Babuška and R. Lipton. Optimal local approximation spaces for generalized finite element methods with application to multiscale problems. Multiscale Modeling \& Simulation, 9:373-406, 2011.

[6] A. T. Barker, C. S. Lee, and P. S. Vassilevski. Spectral upscaling for graph Laplacian problems with application to reservoir simulation. LLNL Report, LLNL-JRNL-693123, 2016. 
[7] D. Boffi, F. Brezzi, and M. Fortin. Mixed finite element methods and applications, volume 44 of Springer Series in Computational Mathematics. Springer Berlin Heidelberg, 2013.

[8] S. Brenner and S. L. Ridgway. The mathematical theory of finite element methods. Texts in Applied Mathematics. Springer-Verlag, New York, second edition, 2002.

[9] S. C. Brenner and L.-Y. Sung. Linear finite element methods for planar linear elasticity. Mathematics of Computation, 59(200):321-338, 1992.

[10] M. Brezina, C. Heberton, J. Mandel, and P. Vanek. An iterative method with convergence rate chosen a priori. UCD CCM Report, 140, 1999.

[11] M. Brezina and P. S. Vassilevski. Smoothed aggregation spectral element agglomeration AMG: SA- $\rho$ AMGe. In Ivan Lirkov, Svetozar Margenov, and Jerzy Waśniewski, editors, Large-Scale Scientific Computing, volume 7116 of Lecture Notes in Computer Science, pages 3-15. Springer Berlin Heidelberg, 2012.

[12] F. Brezzi and M. Fortin. Mixed and hybrid finite element methods, volume 15 of Springer Series in Computational Mathematics. Springer-Verlag, New York, 1991.

[13] M. Buck, O. Iliev, and H. Andrä. Multiscale finite element coarse spaces for the application to linear elasticity. Central European Journal of Mathematics, 11(4):680-701, 2013. 
[14] Z. Cai, R. Lazarov, T. A. Manteuffel, and S. F. McCormick. First-order system least squares for second-order partial differential equations: Part I. SIAM Journal on Numerical Analysis, 31(6):1785-1799, 1994.

[15] V. Calo, Y. Efendiev, J. Galvis, and G. Li. Randomized oversampling for generalized multiscale finite element methods. Multiscale Modeling \& Simulation, 14(1):482-501, 2016.

[16] H. Y. Chan, E. T. Chung, and Y. Efendiev. Adaptive mixed GMsFEM for flows in heterogeneous media. arXiv preprint arXiv:1507.01659, 2015.

[17] T. Chartier, R. Falgout, V.E. Henson, J. Jones, T. Manteuffel, S. McCormick, J. Ruge, and P.S. Vassilevski. Spectral AMGe ( $\rho$ AMGe). SIAM Journal on Scientific Computing, 25:1-26, 2003.

[18] T. Chartier, R. Falgout, V.E. Henson, J. Jones, T. Manteuffel, S. McCormick, J. Ruge, and P.S. Vassilevski. Spectral element agglomerate AMGe. In Domain Decomposition Methods in Science and Engineering XVI., number 55 in Springer, Heidelberg, Lecture Notes in Computational Science and Engineering, pages 515-524, 2007.

[19] G. Chechkin, A. Piatnitski, and A. Shamaev. Homogenization. Methods and applications, volume 234 of Translations of Mathematical Monographs. American Mathematical Society, 2007. 
[20] Z. Chen and T. Y. Hou. A mixed multiscale finite element method for elliptic problems with oscillating coefficients. Mathematics of Computation, 72(242):541-576, 2003.

[21] M. Christie and M. Blunt. Tenth SPE comparative solution project: A comparison of upscaling techniques. SPE Reser. Eval. Eng., 4:308-317, 2001.

[22] C.-C. Chu, I. G. Graham, and T. Y. Hou. A new multiscale finite element method for high-contrast elliptic interface problems. Mathematics of Computation, 79(272):1915-1955, 2010.

[23] E. T. Chung, Y. Efendiev, and S. Fu. Generalized multiscale finite element method for elasticity equations. International Journal on Geomathematics, $5(2): 225-254,2014$.

[24] E. T. Chung, Y. Efendiev, and C. S. Lee. Mixed generalized multiscale finite element methods and applications. Multiscale Modeling \& Simulation, 13(1):338-366, 2015.

[25] E. T. Chung, Y. Efendiev, and W. T. Leung. Generalized multiscale finite element methods for wave propagation in heterogeneous media. Multiscale Modeling \& Simulation, 12(4):1691-1721, 2014.

[26] B. Cockburn and J. Gopalakrishnan. A characterization of hybridized mixed methods for second order elliptic problems. SIAM Journal on Numerical Analysis, 42(1):283-301, 2004. 
[27] B. Cockburn and J. Gopalakrishnan. Error analysis of variable degree mixed methods for elliptic problems via hybridization. Mathematics of Computation, 74(252):1653 1677, 2005.

[28] C. R. Dohrmann. A preconditioner for substructuring based on constrained energy minimization. SIAM Journal on Scientific Computing, 25(1):246-258, 2003.

[29] P. Dostert, Y. Efendiev, and T. Y. Hou. Multiscale finite element methods for stochastic porous media flow equations. Comput. Methods Appl. Math. Engrg., 197:3445-3455, 2008.

[30] L. J. Durlofsky. Numerical calculation of equivalent grid block permeability tensors for heterogeneous porous media. Water Resour. Res., 27:699-708, 1991.

[31] W. E and B. Engquist. Heterogeneous multiscale methods. Comm. Math. Sci., 1(1):87-132, 2003.

[32] W. E, P. Ming, and P. Zhang. Analysis of the heterogeneous multiscale method for elliptic homogenization problems. J. Amer. Math. Soc., 18(1):121-156, 2005.

[33] Y. Efendiev, J. Galvis, and T. Hou. Generalized multiscale finite element methods. Journal of Computational Physics, 251:116-135, 2013. 
[34] Y. Efendiev, J. Galvis, R. Lazarov, and J. Willems. Robust domain decomposition preconditioners for abstract symmetric positive definite bilinear forms. ESIAM : M2AN, 46:1175-1199, 2012.

[35] Y. Efendiev, J. Galvis, G. Li, and M. Presho. Generalized multiscale finite element methods: Oversampling strategies. International Journal for Multiscale Computational Engineering, 12(6):465-485, 2014.

[36] Y. Efendiev, J. Galvis, and P.S. Vassilevski. Spectral element agglomerate algebraic multigrid methods for elliptic problems with high-contrast coefficients. In Domain decomposition methods in science and engineering XIX, volume 78 of Lect. Notes Comput. Sci. Eng., pages 407-414. Springer, Heidelberg, 2011.

[37] Y. Efendiev and T. Hou. Multiscale finite element methods: Theory and applications, volume 4 of Surveys and Tutorials in the Applied Mathematical Sciences. Springer, New York, 2009.

[38] Y. Efendiev, T. Hou, and X.-H. Wu. Convergence of a nonconforming multiscale finite element method. SIAM Journal on Numerical Analysis, 37:888-910, 2000.

[39] R. S. Falk. Finite element methods for linear elasticity. In Daniele Boffi and Lucia Gastaldi, editors, Mixed Finite Elements, Compatibility Conditions, and Applications, volume 1939 of Lecture Notes in Mathematics, pages 159-194. Springer Berlin Heidelberg, 2008. 
[40] J. Galvis and Y. Efendiev. Domain decomposition preconditioners for multiscale flows in high-contrast media. Multiscale Modeling \& Simulation, 8:1461-1483, 2010.

[41] J. Galvis and Y. Efendiev. Domain decomposition preconditioners for multiscale flows in high contrast media. reduced dimension coarse spaces. Multiscale Modeling \& Simulation, 8:1621-1644, 2010.

[42] J. Guzmán. A unified analysis of several mixed methods for elasticity with weak stress symmetry. Journal of Scientific Computing, 44(2):156-169, 2010.

[43] V. E. Henson and U. M. Yang. BoomerAMG: A parallel algebraic multigrid solver and preconditioner. Applied Numerical Mathematics, 41(1):155 - 177, 2002.

[44] R. Hiptmair and J. Xu. Nodal auxiliary space preconditioning in $\mathrm{H}$ (curl) and H(div) spaces. SIAM Journal on Numerical Analysis, 45(6):2483-2509, 2007.

[45] U. Hornung. Homogenization and porous media, volume 6 of Interdisciplinary Applied Mathematics. Springer, New York, 1997.

[46] T. Hou and X.-H. Wu. A multiscale finite element method for elliptic problems in composite materials and porous media. Journal of Computational Physics, 134:169-189, 1997. 
[47] T. Hughes, G. Feijoo, L. Mazzei, and J. Quincy. The variational multiscale method - a paradigm for computational mechanics. Comput. Methods Appl. Mech. Engrg., 166:3-24, 1998.

[48] J. D. Jansen. The egg model - data files, 2013.

[49] J. D. Jansen, R. M. Fonseca, S. Kahrobaei, M. M. Siraj, G. M. Van Essen, and P. M. J. Van den Hof. The egg model -- a geological ensemble for reservoir simulation. Geoscience Data Journal, 1(2):192-195, 2014.

[50] P. Jenny, S. H. Lee, and H. Tchelepi. Multi-scale finite volume method for elliptic problems in subsurface flow simulation. Journal of Computational Physics, 187:47-67, 2003.

[51] J. E. Jones and P. S. Vassilevski. AMGe based on element agglomeration. SIAM Journal on Scientific Computing, 23(1):109-133, 2001.

[52] D. Kalchev, C. S. Lee, U. Villa, Y. Efendiev, and P. S. Vassilevski. Upscaling of mixed finite element discretization problems by the spectral AMGe method. LLNL Report, LLNL-JRNL-676518, 2015.

[53] G. Karypis and V. Kumar. A fast and high quality multilevel scheme for partitioning irregular graphs. SIAM Journal on scientific Computing, 20(1):359-392, 1998. 
[54] H. H. Kim and E. T. Chung. A BDDC algorithm with enriched coarse spaces for two-dimensional elliptic problems with oscillatory and high contrast coefficients. Multiscale Modeling \& Simulation, 13(2):571-593, 2015.

[55] T. V. Kolev and P. S. Vassilevski. Parallel auxiliary space AMG solver for $H$ (div) problems. SIAM Journal on Scientific Computing, 34(6):A3079-A3098, 2012.

[56] I. V. Lashuk and P. S. Vassilevski. On some versions of the element agglomeration AMGe method. Numerical Linear Algebra with Applications, 15:595-620, 2008.

[57] I. V. Lashuk and P. S. Vassilevski. Element agglomeration coarse Raviart-Thomas spaces with improved approximation properties. Numerical Linear Algebra with Applications, 19(2):414-426, 2012.

[58] I. V. Lashuk and P. S. Vassilevski. The construction of the coarse de Rham complexes with improved approximation properties. Computational Methods in Applied Mathematics, 14(2):257-303, 2014.

[59] C. S. Lee and P. S. Vassilevski. Parallel solver for H(div) problems using hybridization and AMG. LLNL Report, LLNL-TR-681025, 2016.

[60] I. Lunati and P. Jenny. Multi-scale finite-volume method for compressible multiphase flow in porous media. Journal of Computational Physics, 216:616-636, 2006. 
[61] K. A. Mardal and R. Winther. Preconditioning discretizations of systems of partial differential equations. Numerical Linear Algebra with Applications, 18(1):140, 2011.

[62] J. E. Pasciak and P. S. Vassilevski. Exact de Rham sequences of spaces defined on macro-elements in two and three spatial dimensions. SIAM Journal on Scientific Computing, 30(5):2427-2446, 2008.

[63] J. E. Roberts and J.-M. Thomas. Mixed and hybrid methods. In Finite Element Methods, Handbook of Numerical Analysis II, (Eds. P. Ciarlet and J. Lions), Elsevier/North Holland, Amsterdam, pages 523-639, 1991.

[64] N. Spillane, V. Dolean, P. Hauret, F. Nataf, C. Pechstein, and R. Scheichl. Abstract robust coarse spaces for systems of PDEs via generalized eigenproblems in the overlaps. Numerische Mathematik, 126(4):741-770, 2014.

[65] N. Spillane, V. Dolean, P. Hauret, F. Nataf, and D. J. Rixen. Solving generalized eigenvalue problems on the interfaces to build a robust two-level FETI method. Comptes Rendus Mathematique, 351(5-6):197 - 201, 2013.

[66] H. A. Tchelepi, P. Jenny, S. H. Lee, and C. Wolfsteiner. An adaptive multiphase multiscale finite volume simulator for heterogeneous reservoirs. SPE J., 12:185195, 2007. 
[67] P. S. Vassilevski. Multilevel Block-Factorization Preconditioners: Matrix-based Analysis and Algorithms for Solving Finite Element Equations. Springer, New York, 1st edition, 2008.

[68] P. S. Vassilevski. Coarse spaces by algebraic multigrid: Multigrid convergence and upscaling error estimates. Adv. Adapt. Data Anal., 3(1-2):229-249, 2011.

[69] P. S. Vassilevski and U. Villa. A block-diagonal algebraic multigrid preconditioner for the Brinkman problem. SIAM Journal on Scientific Computing, 35(5):S3-S17, 2013.

[70] C. Wolfsteiner, S. H. Lee, and H. A. Tchelepi. Modeling of wells in the multiscale finite volume method for subsurface flow simulation. Multiscale Modeling \& Simulation, 5:900-917, 2006.

[71] S. Zampini. PCBDDC: A class of robust dual-primal methods in PETSc. To appear in SIAM Journal on Scientific Computing, 2016.

[72] S. Zampini and D. E. Keyes. On the robustness and prospects of adaptive BDDC methods for finite element discretizations of elliptic PDEs with high-contrast coefficients. In Proceedings of the Platform for Advanced Scientific Computing Conference, PASC '16, pages 6:1-6:13. ACM, New York, USA, 2016. 